\title{
Recent Studies on Cyclic 1,7-Diarylheptanoids: Their Isolation, Structures, Biological Activities, and Chemical Synthesis
}

\author{
Yurngdong Jahng ${ }^{1, *}$ and Jae Gyu Park ${ }^{2}$ (D) \\ 1 College of Pharmacy, Yeungnam University, Gyeongsan 38541, Korea \\ 2 Advanced Bio Convergence Center, Pohang Technopark Foundation, Pohang 37668, Korea; \\ jaegpark@gmail.com \\ * Correspondence: ydjahng@ynu.ac.kr; Tel.: +82-53-810-2821
}

Received: 11 October 2018; Accepted: 22 November 2018; Published: 27 November 2018

\begin{abstract}
Diarylheptanoids are a family of plant secondary metabolites with a 7 carbon skeleton possessing two phenyl rings at the 1- and 7-positions. They can be subdivided into acyclic and cyclic diarylheptanoids where the latter are further divided into meta,meta-bridged biphenyls ([7.0]metacyclophanes) and meta,para-bridged diphenyl ether heptanoids (oxa[7.1]metaparacyclophanes). Since the isolation of curcumin from the rhizomes of turmeric (Curcuma longa) in 1815 which was named curcumin, a variety of diarylheptanoids have been isolated from a number of plant families such as Aceraceae, Actinidiaceae, Betulaceae, Burseraceae, Casuarinaceae, Juglandaceae, Leguminosae, Myricaceae, and Zingiberaceae. Earlier studies on these diarylheptanoids have been summarized on several occasions, of which the main themes only focus on isolation, structure elucidation, and the biological properties of linear types. Only a few have covered cyclic diarylheptanoids and their chemical synthesis has been covered lastly by Zhu et al. in 2000 . The present paper has, therefore, covered recent progress in cyclic diarylheptanoids focusing on the isolation, structural and biological features, and chemical synthesis.
\end{abstract}

Keywords: diarylheptanoid; biphenyl heptanoid; diphenyl ether heptanoid; total synthesis

\section{Introduction}

Diarylheptanoids are a family of plant secondary metabolites with a seven-carbon skeleton possessing two phenyl rings at positions 1 and 7 . They can be subdivided into acyclic diaryheptanoids (type I, linear) and cyclic diarylheptanoids where the latter are further divided into meta,meta-bridged biphenyls (type II, [7.0]metacyclophanes) [1] and meta,para-bridged diphenyl ether heptanoids (type III, oxa[7.1]metaparacyclophanes) [2,3].<smiles>[R]CC(=O)CCc1ccccc1</smiles>

I

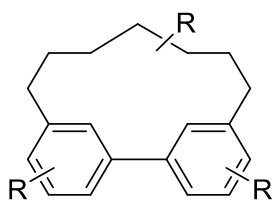

II

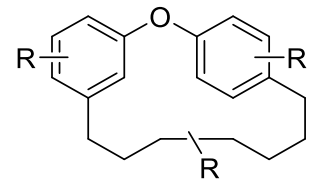

III

Vogel and Pelletier isolated a "yellow coloring-matter" from the rhizomes of turmeric (Curcuma longa) in 1815 and named it curcumin [4], and a century passed before the structure of curcumin by synthesis was defined as the first diarylheptanoid [5]. Since then, a variety of diarylheptanoids have been isolated from a number of plant families including Aceraceae, 
Actinidiaceae, Betulaceae, Burseraceae, Casuarinaceae, Juglandaceae, Leguminosae, Myricaceae, and Zingiberaceae. The extracts of these plants has long been used in traditional oriental medicine in China, India, Japan and Korea as well as ethno-medicine in western countries. Occurrences of three major diarylheptanoids in plants reported so far in the literature are summarized in Table 1 . It should be noted that the Zingiberaceae family has long been the sources of many linear diarylheptanoids including curcumin; no cyclic diarylheptanoids, to the best of our knowledge, have been isolated as yet [6].

Table 1. Occurrence of diarylheptanoids in plants reported in the literature.

\begin{tabular}{|c|c|c|c|c|c|}
\hline \multirow[t]{2}{*}{ Family } & \multirow[t]{2}{*}{ Genus } & \multicolumn{3}{|c|}{ Diarylheptanoids } & \multirow[t]{2}{*}{ Reference(s) } \\
\hline & & $\begin{array}{l}\text { Linear } \\
\text { (Type I) }\end{array}$ & $\begin{array}{l}\text { Biphenyl } \\
\text { (Type II) }\end{array}$ & $\begin{array}{l}\text { Diphenyl Ether } \\
\text { (Type III) }\end{array}$ & \\
\hline Aceraceae & Acer & + & + & + & [7] \\
\hline Actinidiaceae & Clematoclethra & - & - & + & [8] \\
\hline \multirow[t]{6}{*}{ Betulaceae } & Alnus & + & + & + & [9] \\
\hline & Betula & + & + & + & [10] \\
\hline & Corylus & - & + & - & {$[11,12]$} \\
\hline & Carpinus & - & + & - & {$[13]$} \\
\hline & Ostrya & - & + & - & [14] \\
\hline & Ostryopsis & + & + & + & {$[15,16]$} \\
\hline \multirow[t]{2}{*}{ Burseraceae } & Garuga & - & + & + & {$[17,18]$} \\
\hline & Boswellia & - & - & + & [19] \\
\hline Casuarinaceae & Casuarina & - & + & - & [20] \\
\hline \multirow[t]{5}{*}{ Juglandaceae } & Engelhardia & + & - & + & {$[21]$} \\
\hline & Juglans & + & + & + & {$[22,23]$} \\
\hline & Platycarya & - & + & + & [24] \\
\hline & Pterocarya & - & - & + & [25] \\
\hline & Rhoiptelea & + & + & + & {$[26]$} \\
\hline \multirow[t]{2}{*}{ Myricaceae } & Myrica & - & + & + & {$[27,28]$} \\
\hline & Morella & + & + & + & [29] \\
\hline Rubiaceae & Scyphiphora & - & + & - & [30] \\
\hline \multirow[t]{2}{*}{ Zingiberaceae } & Alpinia & + & - & - & [31] \\
\hline & Curcuma & + & - & - & [4] \\
\hline
\end{tabular}

Their unique and characteristic structural features and wide range of biological properties have contributed to their popularity, which has led to continual isolation, evaluation of biological properties, and total synthesis. The earlier studies on diarylheptanoids were summarized by Claeson et al. [32,33], and by Lv and She [34,35], which included all of these three subcategories. Although the two reviews by Claeson et al. covered the general aspects of diarylheptanoids, the reviews by Lv and She focused on their structures, distributions, biological activities and ${ }^{13} \mathrm{C}$-nuclear magnetic resonance (NMR) spectral data of over 400 diarylheptanoids. In addition, additional reviews and papers covering linear diarylheptanoids have appeared recently [36-39], but reviews addressing cyclic diarylheptanoids are very limited. Furthermore, the main themes of the reviews focused on the isolation, structure elucidation, and biological properties while attention to synthesis was somewhat out of scope. Reviews by Keserü and Nógrádi [2] and by Zhu et al. [3] paid attention to the total synthesis of three categories of diarylheptanoids; no additional summaries have been made since then. The present paper has, therefore, covered the recent progress in macrocyclic diarylheptanoids with a focus on their isolation, structural features, biological properties, and synthesis.

\section{Isolation, Structural Features, and Biological Properties}

Diarylheptanoids have been typically isolated from the (inner) stem and the root bark of parent trees and shrubs, from the rhizomes of herbs, and also from the extracts of leaves and twigs of terrestrial plants. Two unusual sources of the green pericarps of walnuts, Juglans regia L. [40], and the nest of the 
paper wasp (Polistes), Nidus vespae [41], have been reported as possible sources of two types of cyclic diarylheptanoids; juglanin A (jugcathanin, 26f) and B (12ba) from the former, and alnusone (4a) and its five congeners from the latter, respectively. Recently, the marine sponge Tedania ignis was found to be a new source of cyclic diarylheptanoids, tedarene A (41a) and B (22) [42].

Owing to the phenolic nature of diarylheptanoids, $\mathrm{MeOH}, \mathrm{MeOH} / \mathrm{CH}_{2} \mathrm{Cl}_{2}$ mixtures, and $\mathrm{EtOH}$ are commonly used for their extraction. However, acetone [43], hexane [44], and toluene [45] have also been used. A recent review paper by Alberti et al. summarized not only the methods and techniques of extraction and isolation, separation and characterization, but also the spectroscopic tools and techniques for structure elucidation of diarylheptanoids in detail [46].

\subsection{Biphenyl Diarylheptanoids}

Biphenyl heptanoids were classified into 4 categories based on their basic structural properties: (1) asadanin and related derivatives, (2) myricanone, myricanol, and related derivatives, (3) garuganins, and (4) miscellaneous.

\subsubsection{Asadanin and Related Derivatives}

Asadanin (1a) was isolated from the $\mathrm{MeOH}$ extract of Ostrya japonica Sarg. (Betulaceae) by Yasue et al. as the first biphenyl heptanoid [14]. They deduced the structure by a series of elegant chemical conversion and degradation study, and later confirmed the structure by NMR spectroscopy [47]. Additional biphenyl heptanoids, such as deoxoasadanin (1b), epiasadanol (1c), isoasadanol (2), and di- and trideoxyasadanin-8-ene (3) were isolated from Ostrya japonica [48]. Recently, Singldinger et al. isolated asadanin (1a) from hazelnuts (Corylus avellana L.) as the main contributor to the bitter off-taste and reported complete analysis and assignment of all the proton and carbon resonances of the molecule [49].<smiles>[X]C(CC(O)Cc1ccc(O)c(-c2cc(CC(O)O)ccc2O)c1)C(O)CO</smiles>

1a (asadanin, $X=0)$

b (deoxoasadanin, $\mathrm{X}=\mathrm{H}, \mathrm{H}$ )

c [epiasadanol, $\mathrm{X}=\mathrm{H}, \mathrm{OH}(R)$ ]<smiles>OCC(O)Cc1ccc(O)c(-c2cc(CCC(O)O)ccc2O)c1</smiles>

2 (isoasadanol)<smiles>[X]C(CC)Cc1ccc(O)c(-c2cc(CC=CC(=O)CC(C)C)ccc2O)c1</smiles>

3a (Dideoxyasadanin-8-ene, $\mathrm{X}=\mathrm{OH}$ ) b (Trideoxyasadanin-8-ene, $X=H$ )

Nomura and Tokoroyama reported a series of related cyclic biphenyl diarylheptanoids, alnusone (4a), alnusonol (5a), and alnusoxide (6) from Alnus japonica (Betulaceae) [50]. Later, an alnusdiol (7a) was additionally isolated from the same source [51]. It should be noted that the absolute configuration of alnusdiol (7a) upon biphenyl axis was determined by X-ray and $\mathrm{CD}$ analysis to be $\mathrm{a} S$ and two additional chiral centers in the heptane skeleton were determined to be $3 S, 5 S$ [52]. Alnusone and two other related compounds $4 \mathbf{b}, \mathbf{c}$ were also isolated from Corylus sieboldiana [53]. The same group isolated three more related heptanoids $\mathbf{5 a}, \mathbf{8}$, and $\mathbf{9}$ from C. sieboldiana [54]. The isolation of diarylheptanoid $\mathbf{8}$ is somewhat surprising due to the presence of the isopropylidene group as a partial structure at the side chain even though its precursor $5 \mathbf{c}$ has been isolated at the same time. Recently, the related compound $\mathbf{5 d}$ (see Table 2), which was also isolated from Alnus japonica, showed very potent anti-adipogenic activity [55]. The diarylheptanoid alnusdiol (7a) was also isolated from Casuarina junghuhniana (Casuarinaceae), along with casuarinondiol (5b) [20] and Betula maximowicziana, along with $5 \mathbf{b}$ and an alnusdiol glycoside (7b) [56] later. The casuarinondiol (5b) was additionally isolated from Scyphiphora hydrolphyllaceae (Rubiaceae) [30]. 

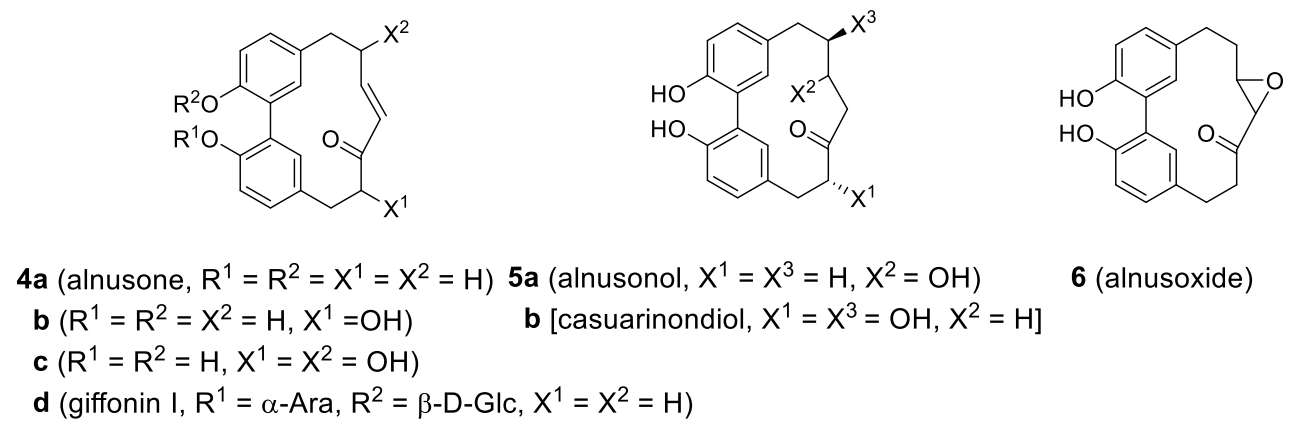

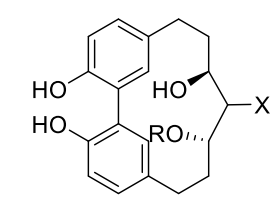

7a (alnusdiol, $R=X=H)$

b $(R=\beta$-D-Glc, $X=H)$

c $(\mathrm{R}=\mathrm{H}, \mathrm{X}=\mathrm{OH})$

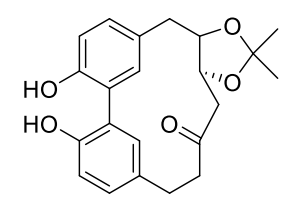

8

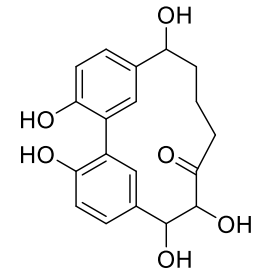

9

A series of the related derivatives of alnusonol (5a) and alnusdiol (7a) are summarized in Table 2. It should be noted that the biphenyl heptanoid $5 \mathbf{j}$ has a unique substitution pattern on the biphenyl ring and has never been isolated elsewhere.

Structurally related systems have been isolated and named as aceroside XI, acerogenins E and K, and giffonins. Diarylheptanoid $\mathbf{5 k}$ was isolated from Acer nikoense (Aceraceae) and named as aceroside XI [57], of which hydrolysis afforded the known heptanoid, acerogenin E (51), the first synthetic biphenyl heptanoid previously reported from the hydrogenation of alnusone (4a) [50] and later isolated from the inner bark of Betula ermanii [58], along with acerogenin K (7h) [59]. Acerogenin E was also isolated from Betula platyphylla var. japonica [60] and Acer nikoense [59]. There are 11 species of Betula in Japan, in which the constituents vary; acerogenin E derivatives 10a,b as the major biphenyl heptanoids in B. davurica [61]. Compound 10b was also isolated from leaves of B. platyphylla [62] and showed promising leishmanicidal activity $\left(\mathrm{IC}_{50}=28 \mu \mathrm{g} / \mathrm{mL}\right.$ ) [63]. In addition, the biphenyl heptanoid 10c was isolated from the leaves and fruits of Rhoiptelea chiliantha (Rhoipteleaceae) [26].

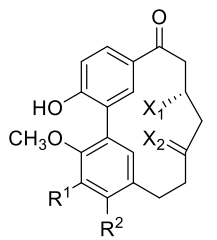

10a $\left(R^{1}=R^{2}=H, X^{1}=H, X^{2}=O\right)$

b $\left(R^{1}=H, R^{2}=O_{C H}, X^{1}=H, X^{2}=O\right)$

c $\left(\mathrm{R}^{1}=\mathrm{OCH}_{3}, \mathrm{R}^{2}=\mathrm{H}, \mathrm{X}^{1}=\mathrm{OH}, \mathrm{X}^{2}=\mathrm{H}, \mathrm{H}\right)$

Recently, two series of biphenyl giffonins $\mathrm{M}, \mathrm{N}$, and T (5e-g) and giffonins $\mathrm{L}, \mathrm{O}, \mathrm{P}$, and $\mathrm{U}(\mathbf{7 d}-\mathbf{g})$ were isolated from hazelnuts (Corylus avellana) [11,12] and are summarized in Table 2. The relative configurations of giffonins were established by a combined quantum mechanical $(\mathrm{QM}) / \mathrm{NMR}$ approach, comparing the experimental ${ }^{13} \mathrm{C} /{ }^{1} \mathrm{H}$ NMR chemical shift and the related predicted values. Carpinontriol B (5i) and giffonin U (7g) at $40 \mu \mathrm{g} /$ disk caused the formation of zones of inhibition against not only Gram-positive bacteria such as Bacillus cereus and Staphylococcus aureus but also Gram-negative Escherichia coli and Pseudomonas aeruginosa [64]. 
Table 2. Derivatives of alnusonol (5a) and alnusdiol (7a), and their stereochemistry.

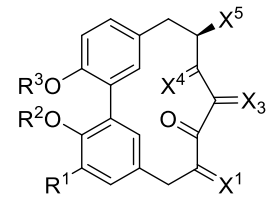

5

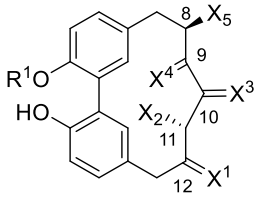

7

\begin{tabular}{|c|c|c|c|c|c|c|c|c|c|c|}
\hline & $\mathbf{R}^{1}$ & $\mathbf{R}^{2}$ & $\mathbf{R}^{3}$ & $X^{1}$ & $\mathrm{X}^{2}$ & $x^{3}$ & $X^{4}$ & $x^{5}$ & Chiral Axis & Reference and Biological Activity \\
\hline alnusonol (5a) & $\mathrm{H}$ & $\mathrm{H}$ & $\mathrm{H}$ & $\mathrm{H}, \mathrm{H}$ & - & $\mathrm{H}, \mathrm{H}$ & $\mathrm{H}, \mathrm{OH}(S)$ & $\mathrm{H}$ & & $\alpha$-glucosidase inhibitor [65] \\
\hline alnusdiol (7a) & $\mathrm{H}$ & $\mathrm{H}$ & $\mathrm{H}$ & $\mathrm{H}, \mathrm{H}$ & $\mathrm{OH}(S)$ & $\mathrm{H}, \mathrm{H}$ & $\mathrm{H}, \mathrm{OH}(\mathrm{S})$ & $\mathrm{H}$ & aS & [51] \\
\hline $5 c$ & $\mathrm{H}$ & $\mathrm{H}$ & $\mathrm{H}$ & $\mathrm{H}, \mathrm{H}$ & - & $\mathrm{H}, \mathrm{H}$ & $\mathrm{H}, \mathrm{OH}$ & $\mathrm{OH}$ & & {$[53]$} \\
\hline $5 d$ & $\mathrm{H}$ & $\mathrm{H}$ & $\mathrm{H}$ & $\mathrm{H}, \mathrm{H}$ & - & $\mathrm{H}, \mathrm{OH}$ & $\mathrm{O}$ & $\mathrm{H}$ & & anti-adipogenic activity [55] \\
\hline giffonin $\mathrm{L}^{\mathrm{a}}(\mathbf{7 d})$ & $\mathrm{H}$ & $\mathrm{H}$ & $\mathrm{H}$ & $\mathrm{H}, \mathrm{OH}(S)$ & $\mathrm{OH}(S)$ & $\mathrm{H}, \mathrm{OH}(R)$ & $\mathrm{H}, \mathrm{H}$ & $\mathrm{OH}(S)$ & & [12] \\
\hline giffonin M (5e) & $\mathrm{H}$ & $\mathrm{H}$ & $\mathrm{H}$ & $\mathrm{H}, \mathrm{H}$ & - & $\mathrm{H}, \mathrm{OH}(S)$ & $\mathrm{H}, \mathrm{H}$ & $\mathrm{OH}(S)$ & & [12] \\
\hline giffonin N (5f) & $\mathrm{H}$ & $\mathrm{H}$ & $\mathrm{H}$ & $\mathrm{H}, \mathrm{H}$ & - & $\mathrm{H}, \mathrm{OH}(S)$ & $\mathrm{H}, \mathrm{H}$ & $O-\beta$-D-Glc $(S)$ & & [12] \\
\hline giffonin $\mathrm{O}(7 \mathbf{e})$ & $\mathrm{H}$ & $\mathrm{H}$ & $\mathrm{H}$ & $\mathrm{H}, \mathrm{OH}(R)$ & $\mathrm{H}$ & $\mathrm{O}$ & $\mathrm{H}, \mathrm{OH}(R)$ & $\mathrm{OH}(S)$ & & [12] \\
\hline giffonin $\mathrm{P}^{\mathrm{a}}(\mathbf{7 f})$ & $\mathrm{H}$ & $\mathrm{H}$ & $\mathrm{H}$ & $\mathrm{H}, \mathrm{OH}(R)$ & $\mathrm{OH}(S)$ & $\mathrm{H}, \mathrm{OH}$ & $\mathrm{H}, \mathrm{OH}(\mathrm{S})$ & $\mathrm{OH}(S)$ & & [12] \\
\hline giffonin T (5g) & $\mathrm{H}$ & $\mathrm{R}^{\prime \mathrm{b}}$ & $\mathrm{H}$ & $\mathrm{H}, \mathrm{H}$ & - & $\mathrm{H}, \mathrm{OH}(R)$ & $\mathrm{H}, \mathrm{OH}(S)$ & $\mathrm{OH}(R)$ & aS & [64] \\
\hline giffonin U (7g) & $\mathrm{H}$ & $\mathrm{H}$ & $\mathrm{H}$ & $\mathrm{O}$ & $\mathrm{OH}(R)$ & $\mathrm{H}, \mathrm{OH}(R)$ & $\mathrm{H}, \mathrm{OH}(R)$ & $\mathrm{OH}(R)$ & aS & [64] \\
\hline carpinontriol A (5h) & $\mathrm{H}$ & $\mathrm{H}$ & $\mathrm{H}$ & $\mathrm{H}, \mathrm{OH}(R)$ & - & $\mathrm{H}, \mathrm{H}$ & $\mathrm{H}, \mathrm{OH}(S)$ & $\mathrm{OH}(R)$ & & {$[13]$} \\
\hline carpinontriol B (5i) & $\mathrm{H}$ & $\mathrm{H}$ & $\mathrm{H}$ & $\mathrm{H}, \mathrm{H}$ & - & $\mathrm{H}, \mathrm{OH}(R)$ & $\mathrm{H}, \mathrm{OH}(S)$ & $\mathrm{OH}(R)$ & aS & [13], inhibit lipid peroxidation [64] \\
\hline $5 \mathbf{j}$ & $\mathrm{OH}$ & $\mathrm{CH}_{3}$ & $\mathrm{H}$ & $\mathrm{H}, \mathrm{H}$ & - & $\mathrm{H}, \mathrm{H}$ & $\mathrm{H}, \mathrm{H}$ & $\mathrm{H}$ & & $\begin{array}{c}\text { [56], leishmanicidal } \\
\left(\mathrm{IC}_{50}=17 \mathrm{mg} / \mathrm{mL}\right)[63]\end{array}$ \\
\hline aceroside $X I^{c}(5 \mathbf{k})$ & $\mathrm{H}$ & $\mathrm{H}$ & $\mathrm{R}^{\prime \mathrm{b}}$ & $\mathrm{H}, \mathrm{H}$ & - & $\mathrm{H}, \mathrm{H}$ & $\mathrm{H}, \mathrm{H}$ & $\mathrm{H}$ & & [66] \\
\hline acerogenin $E^{d}(51)$ & $\mathrm{H}$ & $\mathrm{H}$ & $\mathrm{H}$ & $\mathrm{H}, \mathrm{H}$ & - & $\mathrm{H}, \mathrm{H}$ & $\mathrm{H}, \mathrm{H}$ & $\mathrm{H}$ & & $\begin{array}{l}\text { NO production inhibitor } \\
\quad\left(\mathrm{IC}_{50}=24 \mu \mathrm{M}\right)[67]\end{array}$ \\
\hline acerogenin $K(7 h)$ & $\mathrm{H}$ & $\mathrm{H}$ & $\mathrm{H}$ & $\mathrm{H}, \mathrm{H}$ & $\mathrm{OH}$ & $\mathrm{H}, \mathrm{H}$ & $\mathrm{H}, \mathrm{H}$ & $\mathrm{H}$ & & $\begin{array}{l}\text { NO production inhibitor } \\
\quad\left(\mathrm{IC}_{50}=24 \mu \mathrm{M}\right)[67]\end{array}$ \\
\hline ostryopsitriol (7i) & $\mathrm{H}$ & $\mathrm{H}$ & $\mathrm{H}$ & $\mathrm{H}, \mathrm{OH}$ & $\mathrm{OH}$ & $\mathrm{H}, \mathrm{H}$ & $\mathrm{H}, \mathrm{OH}$ & $\mathrm{H}$ & & {$[16]$} \\
\hline betulatetraol (7j) & $\mathrm{H}$ & $\mathrm{H}$ & $\mathrm{H}$ & $\mathrm{H}, \mathrm{OH}$ & $\mathrm{OH}$ & $\mathrm{H}, \mathrm{H}$ & $\mathrm{H}, \mathrm{OH}$ & $\mathrm{OH}$ & & [43] \\
\hline
\end{tabular}

${ }^{a}$ Giffonin A-K, $\mathrm{O}, \mathrm{R}$, and $\mathrm{S}$ are diphenyl ether heptanoids (vide infra). ${ }^{\mathrm{b}} \mathrm{R}^{\prime}=\beta$-D-glucopyranosyl. ${ }^{\mathrm{c}}$ Aceroside I-X are cyclic diphenyl ether heptanoids (vide infra).

d Acerogenins A-D, F, H-J, L, and M are cyclic diphenyl ether heptanoids (vide infra). 


\subsubsection{Myricanone, Myricanol and Related Derivatives}

The second series of biphenyl diarylheptanoids, myricanone (11a) and myricanol (12a) were isolated from Myrica nagi (Myricaceae) [68-70]. Myricanone has a variety of biological activities, which have been summarized in Table 3, whereas myricanol has an anti-tau activity thus having anti-Alzheimer's disease activity. It has been figure out that the $a S, 11 R$ enantiomer $[(-)-\mathbf{1 2 a a}-(\mathrm{a} S)(11 R)$, vide infra] is responsible for the majority of tau-lowering activity [71].<smiles>[X]CCCCc1cc(-c2cc(CCC([X])=[V])ccc2O)c(OC)c(OC)c1O</smiles>

11a (myricanone, $\mathrm{X}=\mathrm{O}$ ) 12a (myricanol, $\mathrm{X}=\mathrm{H}, \mathrm{OH}$ )<smiles>[R]CCCc1ccc(O)c(-c2cc(CCCC(O)C[R])c(O)c(OC)c2OC)c1</smiles>

$(-)-12 \mathbf{a a}-(\mathrm{a} S)(11 R)$<smiles></smiles>

13

The absolute configuration at C-11 of myricanol (12aa) was determined by an X-ray crystal structure on 16-bromomyricanol (13), prepared by brominating myricanol, and found to be $\mathrm{a} R[69,70]$. Although the absolute configuration of secondary alcohol at C-11 was assigned as $S$, the authors did not mention about the atropisomerism [72,73] of the molecule developed by biphenyl axis. However, the configuration of the biphenyl axis of 16-bromomyricanol could be readily assigned to $P$ (more likely aR) configuration by carefully analyzing X-ray crystal structure. Myricanol has also been isolated from M. gale [74], M. rubra [28], M. esculenta [75], and M. cerifera [44], and Morella salicifolia [29] as well. Later, an extensive NMR study revised a previous ${ }^{13} \mathrm{C}$-assignment and X-ray analysis study of $( \pm)$-myricanol that confirmed the structure [44]. In addition, additional derivatives of myricanone and myricanol as well as their glycosides isolated so far reported, are summarized in Tables 3 and 4. 
Table 3. The derivatives of myricanone (11a) and their biological properties.

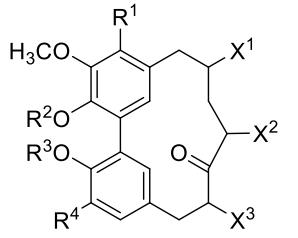

11

\begin{tabular}{|c|c|c|c|c|c|c|c|c|}
\hline Compound & $\mathbf{R}^{1}$ & $\mathbf{R}^{2}$ & $\mathbf{R}^{3}$ & $\mathbf{R}^{4}$ & $X^{1}$ & $X^{2}$ & $X^{3}$ & Reference(s) and Biological Properties \\
\hline 11a (myricanone) & $\mathrm{OH}$ & $\mathrm{CH}_{3}$ & $\mathrm{H}$ & $\mathrm{H}$ & $\mathrm{H}$ & $\mathrm{H}$ & $\mathrm{H}$ & $\begin{array}{c}\text { cytotoxicity }[76,77], \text { cell apoptosis [78], anti-inflammatory } \\
\text { in iNOS assay }\left(\mathrm{IC}_{50}=1.0 \mu \mathrm{M}\right)[79], \text { radical scavenging } \\
\left(\mathrm{IC}_{50}=19.6 \mu \mathrm{M}\right)[79], \text { lowering protein tau level [71], } \\
\text { chemo-preventive }^{\mathrm{a}}\left(\mathrm{IC}_{50}=320\right)[80]\end{array}$ \\
\hline $11 b$ & $O-\beta-G l c$ & $\mathrm{CH}_{3}$ & $\mathrm{H}$ & $\mathrm{H}$ & $\mathrm{H}$ & $\mathrm{H}$ & $\mathrm{H}$ & DPPH radical scavenging activity $(49.09 \%)$ [81] \\
\hline 11c (alnuheptanoid B) & $\begin{array}{c}O-\beta-G l c \\
\text { O- } \beta-G l c\end{array}$ & $\mathrm{CH}_{3}$ & $\mathrm{COCH}_{3}$ & $\mathrm{H}$ & $\mathrm{H}$ & $\mathrm{H}$ & $\mathrm{H}$ & DPPH radical scavenging activity $(41.16 \%)$ [81] \\
\hline 11d (saliciclaireone A) & HROS & $\mathrm{CH}_{3}$ & $\mathrm{H}$ & $\mathrm{H}$ & $\mathrm{H}$ & $\mathrm{H}$ & $\mathrm{H}$ & [29] \\
\hline 11e (saliciclaireone C) & $\mathrm{OCH}_{3}$ & $\mathrm{H}$ & HRO & $\mathrm{H}$ & $\mathrm{H}$ & $\mathrm{H}$ & $\mathrm{H}$ & [29] \\
\hline $11 f$ & & $\mathrm{CH}_{3}$ & $\mathrm{H}$ & $\mathrm{H}$ & $\mathrm{H}$ & $\mathrm{H}$ & $\mathrm{H}$ & [29] \\
\hline myricananin C (11g) & $\mathrm{H}$ & $\mathrm{H}$ & $\mathrm{H}$ & $\mathrm{H}$ & $\mathrm{H}$ & $\mathrm{H}$ & $\mathrm{H}$ & NO release inhibition $\left(\mathrm{IC}_{50}=64.51 \mu \mathrm{M}\right)$ [82] \\
\hline myricananin E (11h) & $\mathrm{OH}$ & $\mathrm{CH}_{3}$ & $\mathrm{H}$ & $\mathrm{OH}$ & $\mathrm{H}$ & $\mathrm{H}$ & $\mathrm{OCH}_{3}$ & [82] \\
\hline myricananone (11i) & $\mathrm{OH}$ & $\mathrm{CH}_{3}$ & $\mathrm{H}$ & $\mathrm{H}$ & $\mathrm{OH}$ & $\mathrm{H}$ & $\mathrm{H}$ & {$[83]$} \\
\hline $11 \mathrm{j}$ & $\mathrm{H}$ & $\mathrm{CH}_{3}$ & $\mathrm{H}$ & $\mathrm{H}$ & $\mathrm{H}$ & $\mathrm{H}$ & $\mathrm{H}$ & [84], anti-tubercular activity $\left(\mathrm{IC}_{50}=25.8 \mu \mathrm{g} / \mathrm{mL}\right)[79]$ \\
\hline $11 \mathrm{k}$ & $\mathrm{OH}$ & $\mathrm{CH}_{3}$ & $\mathrm{H}$ & $\mathrm{H}$ & $\mathrm{H}$ & $\mathrm{H}$ & $\mathrm{OH}$ & anti-tubercular activity $\left(\mathrm{IC}_{50}=35.8 \mu \mathrm{g} / \mathrm{mL}\right)[79]$ \\
\hline
\end{tabular}

a Value is the concentration (Mol ratio/TPA) needed to inhibit $50 \%$ of positive control activated with TPA. 
Table 4. The derivatives of myricanol (12a) and their biological properties.

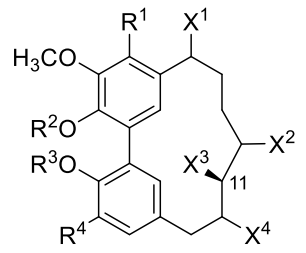

12

\begin{tabular}{|c|c|c|c|c|c|c|c|c|c|}
\hline Compound & $\mathbf{R}^{1}$ & $\mathbf{R}^{2}$ & $\mathbf{R}^{3}$ & $\mathbf{R}^{4}$ & $x^{1}$ & $x^{2}$ & $X^{3}$ & $X^{4}$ & Reference(s) and Biological Properties \\
\hline myricanol (12a) & $\mathrm{OH}$ & $\mathrm{CH}_{3}$ & $\mathrm{H}$ & $\mathrm{H}$ & $\mathrm{H}$ & $\mathrm{H}$ & $\mathrm{OH}(S)$ & $\mathrm{H}$ & $\begin{array}{l}\text { [68], radical scavenging }\left(\mathrm{IC}_{50}=22.3 \mu \mathrm{M}\right)[79] \\
\text { anti-Alzheimer's disease by lowering tau level [71] }\end{array}$ \\
\hline juglanin B (12ba) & $\mathrm{H}$ & $\mathrm{H}$ & $\mathrm{H}$ & $\mathrm{H}$ & $\mathrm{H}$ & $\mathrm{H}$ & $\mathrm{OH}(S)$ & $\mathrm{H}$ & cytotoxic (HT-29) [40] \\
\hline salicireneol A (12c) & $\mathrm{H}$ & $\beta$-Glc & $\mathrm{H}$ & $\mathrm{H}$ & $\mathrm{H}$ & $\mathrm{H}$ & $\mathrm{OH}(S)$ & $\mathrm{H}$ & [29] \\
\hline salicireneol B (12d) & $\mathrm{H}$ & $\mathrm{H}$ & $\beta$-Glc & $\mathrm{OH}$ & $\mathrm{H}$ & $\mathrm{H}$ & $\mathrm{OH}(S)$ & $\mathrm{H}$ & {$[29]$} \\
\hline myricananin A (12e) & $\mathrm{H}$ & $\mathrm{H}$ & $\mathrm{H}$ & $\mathrm{H}$ & $\mathrm{H}$ & $\mathrm{H}$ & $\mathrm{OH}(S)$ & $\mathrm{OH}(S)$ & NO release inhibitor $\left(\mathrm{IC}^{50}=45.32 \mu \mathrm{M}\right)[82]$ \\
\hline myricananin B (12f) & $\mathrm{OH}$ & $\mathrm{CH}_{3}$ & $\mathrm{H}$ & $\mathrm{H}$ & $\mathrm{H}$ & $\mathrm{H}$ & $\mathrm{OH}(S)$ & $\mathrm{OH}(S)$ & {$[82]$} \\
\hline myricananin F (12g) & $\mathrm{H}$ & $\mathrm{H}$ & $\mathrm{H}$ & $\mathrm{H}$ & $\mathrm{H}$ & $\mathrm{OH}$ & $\mathrm{H}$ & $\mathrm{H}$ & [85] \\
\hline myricananin $G(\mathbf{1 2 h})$ & $\mathrm{OH}$ & $\mathrm{CH}_{3}$ & $\mathrm{CH}_{3}$ & $\mathrm{H}$ & $\mathrm{H}$ & $\mathrm{H}$ & $\mathrm{OH}(S)$ & $\mathrm{OH}(R)$ & [85] \\
\hline myricananin H (12i) & $\mathrm{OCH}_{3}$ & $\mathrm{CH}_{3}$ & $\mathrm{CH}_{3}$ & $\mathrm{H}$ & $\mathrm{H}$ & $\mathrm{H}$ & $\mathrm{OH}(S)$ & $\mathrm{OH}(S)$ & [85] \\
\hline salicimeckol (12j) & $O-\beta$-Glc & $\mathrm{CH}_{3}$ & $\mathrm{H}$ & $\mathrm{H}$ & $\mathrm{OH}$ & $\mathrm{H}$ & $\mathrm{OH}(S)$ & $\mathrm{H}$ & {$[29]$} \\
\hline $12 \mathrm{k}$ & $O-\beta-G l c$ & $\mathrm{CH}_{3}$ & $\mathrm{H}$ & $\mathrm{H}$ & $\mathrm{H}$ & $\mathrm{H}$ & $\mathrm{OH}(S)$ & $\mathrm{H}$ & anti-allergic activity [86] \\
\hline 121 & O- $\alpha$-L-araf & $\mathrm{CH}_{3}$ & $\mathrm{H}$ & $\mathrm{H}$ & $\mathrm{H}$ & $\mathrm{H}$ & $\mathrm{OH}(S)$ & $\mathrm{H}$ & $\begin{array}{l}\text { [87], DPPH radical scavenging }\left(\mathrm{IC}_{50}=6.8 \mu \mathrm{M}\right) \text { [88], } \\
\text { SOD-like activity }\left(\mathrm{IC}_{50}=90.5 \mu \mathrm{g} / \mathrm{mL}\right)[89]\end{array}$ \\
\hline $12 \mathrm{~m}$ & HRO ${ }_{\mathrm{OH}} \mathrm{O}$ & $\mathrm{CH}_{3}$ & $\mathrm{H}$ & $\mathrm{H}$ & $\mathrm{H}$ & $\mathrm{H}$ & $\mathrm{OH}(S)$ & & [90] \\
\hline $12 n$ & O-B-D-Glc & $\mathrm{CH}_{3}$ & $\mathrm{H}$ & $\mathrm{H}$ & $\mathrm{H}$ & $\mathrm{H}$ & $\mathrm{OH}(S)$ & & [87] \\
\hline
\end{tabular}


It should be noted that a series of myricananins $\mathrm{A}-\mathrm{H}(\mathbf{1 2 e}, \mathbf{1 2 f}, \mathbf{1 1 g}, \mathbf{1 4}, \mathbf{1 1 h}$, and $\mathbf{1 2 g}-\mathbf{i})$ were also isolated from Myrica nana and showed inhibitory activity $\left(\mathrm{IC}_{50}=45-63 \mu \mathrm{M}\right)$ on nitric oxide release in LPS-activated peritoneal macrophages [82].

A compound with a close structural relationship to myricanol (12a) in structure, was isolated from Juglans regia and named as juglanin B (12ba) [40]; the structure of this was originally proposed in error [91]. Subsequently, two esters of sulfuric acid such as myricanol 11-sulfate (12ab) and juglanin B 11-sulfate (12bb), and related glycosides (12j-n) were isolated from M. rubra [89]. The juglanin B (12ba) showed cytotoxic activity against human hepatoma Hep G2 cells [40].

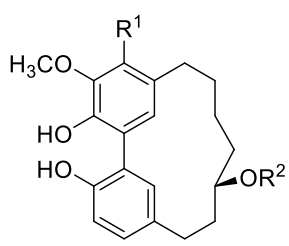

12ab (myricanol 11-sulfate, $\mathrm{R}^{1}=\mathrm{OH}, \mathrm{R}^{2}=\mathrm{SO}_{3} \mathrm{H}$ bb (juglanin $B$ 11-sulfate, $\mathrm{R}^{1}=\mathrm{H}, \mathrm{R}^{2}=-\mathrm{SO}_{3} \mathrm{H}$ )

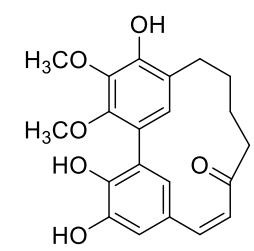

14 (myricananin D)

Four additional related heptanoids 15 were isolated from Myrica gale [92]. The heptanoid 15a, known as porson, was first isolated from the same plant by Anthonsen et al. [74], whose structure proposed was revised 20 years later by Nagai et al. [92]. Saliciclaireone B (16), a regioisomer of 11d (saliciclaireone A), was also isolated from Morella salicifolia [29]. Porson showed promising anti-tuberculosis activity, with an MIC value of $40 \mu \mathrm{g} / \mathrm{mL}$ [79].

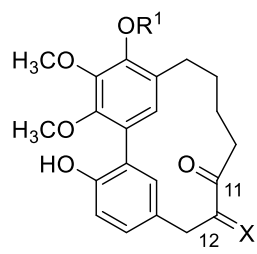

15a (porson, $\mathrm{R}^{1}=\mathrm{CH}_{3}, \mathrm{X}=\mathrm{H}, \mathrm{OH}$ )

b (12-dehydroporson, $\mathrm{R}^{1}=\mathrm{CH}_{3}, \mathrm{X}=\mathrm{O}$ )

c $\left(\mathrm{R}^{1}=\mathrm{CH}_{3}, \mathrm{X}=\mathrm{H}, \mathrm{OH}\right)$

d (myricatomenoside, $\mathrm{R}^{1}=\beta$-D-Glu, $X=\mathrm{H}, \mathrm{OH}$ )

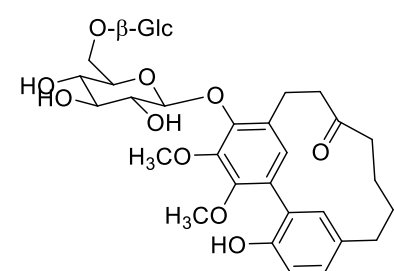

16 (saliciclaireone B)

\subsubsection{Garuganins}

A series of biphenyl heptanoids with a heptene skeleton have also been reported. Garuganin II (17a) [93], garuganin V (17b) [17], and 6'-hydroxygaruganin V (18) [18], were isolated from Garuga pinnata as biphenyl heptanoids in the garuganin series I-VII (vide infra). The X-ray structures of garuganin II [93] and V [94] showed that the (Z)-heptene is the basic skeleton, unlike the (E)-heptene of alnusone series. As it is thought that adapting $(E)$-alkene geometry to accommodate a bulky methoxy substituent inside the cyclic diarylheptanoids generally imposes substantial strain to the ring system, the $(E)$-heptene structure in $6^{\prime}$-hydroxygaruganin $V(\mathbf{1 8})$, proposed by Ara et al. is somewhat surprising. Indeed, the minimized total energies of the two $(Z)-\mathbf{1 8}$ with $(E)$-hepta-6-en-5-one skeleton and $(E)-\mathbf{1 8}$ with (Z)-hepta-6-en-5-one skeleton calculated by MM2 are $166.8 \mathrm{Kcal} /$ mole and $45.6 \mathrm{Kcal} / \mathrm{mole}$, respectively, reflecting the fact that $\mathbf{1 8}$ with (Z)-heptene moiety is much more stable. The structure of $\mathbf{1 8}$, thus, remained to be clarified. Garuganin V showed strong bactericidal activity against Bacillus sereus and B. subtilus as well as Gram-negative Salmonella enterica paratyphi [95]. 


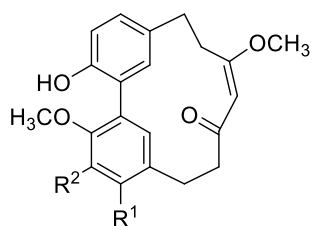

17a (garuganin II, $R^{1}=\mathrm{OCH}_{3}, \mathrm{R}^{2}=\mathrm{H}$ ) b (garuganin $\mathrm{V}, \mathrm{R}^{1}=\mathrm{R}^{2}=\mathrm{H}$ )

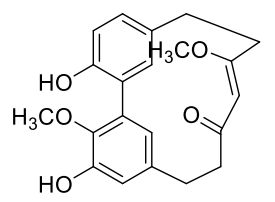

(Z)-18 (6'-hydroxygaruganin V)<smiles>CO/C(=C/C(=O)CCc1cc(O)c(OC)c(-c2cc(CCC(=O)O)ccc2O)c1)CO</smiles>

(E)-18

\subsubsection{Miscellaneous}

The structurally novel diarylheptanoid actinidione (19) was isolated from the leaves and twigs of Clematoclethra actinidioides (Actinidiaceae) [8]. It is somewhat surprising that $\mathbf{1 9}$ is the first biphenyl heptanoids with a [7.0]orthometacyclophane skeleton and a 1,4-benzoquinone, and showed promising cytotoxicity $\left(\mathrm{GI}_{50}=16.03-32.58 \mu \mathrm{g} / \mathrm{mL}\right)$ against Lun-06, Neu-04, Bre-04 cell lines. This compound was later isolated from the bark of Myrica nana [82] along with two additional new [7.0]metacyclophanes, rubanol (20) [96] and nanaone (21) [97], in which one benzene ring was oxidized to 1,4-benzoquinone. As one may expect from the 1,4-benzoquinone moiety, 19 showed strong antioxidant activity $\left(\mathrm{IC}_{50}=7.9 \mu \mathrm{g} / \mathrm{mL}\right)$ against superoxide dismutase (SOD) [89].<smiles>COC1=C(OC)C(=O)C(CCCCC(O)CCc2ccc(O)c(C3=C(C)C(OC)=C(OC)C(=O)C3=O)c2)=C(O)C1=O</smiles>

19 (actinidione)<smiles></smiles>

20 (rubanol)<smiles>COC1=C(O)C(=O)C(=O)C(OC)=C1CCCC/C=C\Cc1ccc(O)c(C2=C(OC)C(=O)C(OC)=C(c3ccccc3)C2=O)c1</smiles>

21 (nanaone)

Recently, biphenyl heptanoid tedarene B (22) was isolated from marine sponge Tedania ignis [42]. Tedarene B has a unique structure with three chiral elements: one stereogenic carbon (C-9), one chiral axis through the biphenyl unit, and one chiral plane in the double bond, yielding 4 potential atropisomers for each of the two configurational stereoisomers at C-9; that is, 4 possible diastereomeric atropisomers of the configuration stereoisomer $9-S$, i.e., $\left(9 S, 2 S_{a}, 10 S_{p}\right.$ or $\left.9 S, 2 M, 10 M\right),\left(9 S, 2 S_{a}, 10 R_{p}\right.$ or $9 S, 2 M, 10 P),\left(9 S, 2 R_{\mathrm{a}}, 10 S_{\mathrm{p}}\right.$ or $\left.9 S, 2 P, 10 M\right)$, and $\left(9 S, 2 R_{\mathrm{a}}, 10 R_{\mathrm{p}}\right.$ or $\left.9 S, 2 P, 10 P\right)$ [98]. Among them, 22a $\left(9 S, 2 S_{a}, 10 S_{p}\right)$ was isolated, but the ${ }^{1} \mathrm{H}$ NMR showed a $4: 1$ equilibrium mixture of $2 \mathbf{2 a}$ and $\mathbf{2 2} \mathbf{b}$ after $24 \mathrm{~h}$ due to the conformational isomerism of the alkene moiety as shown below.<smiles>C=CCc1ccc(O)c(-c2c(S(=O)(=O)O)ccc3c2C2CCC3C2)c1</smiles>

22a (tedarene B: $9 S, 2{ }_{\mathrm{a}} S, 10_{\mathrm{p}} S$ )

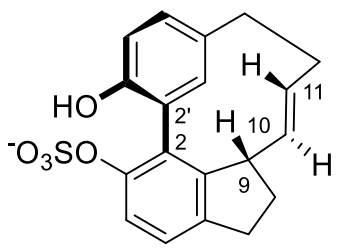

22b (tedarene B: $9 S, 2{ }_{a} S, 10_{p} R$ )

\subsection{Diphenyl Ether Diarylheptanoids}

The diphenyl ether diarylheptanoids were categorized into three major groups based on their skeletal properties: (1) acerogenins and acerosides, (2) garuganins and garugamblins, and (3) miscellaneous. 


\subsubsection{Acerogenins and Acerosides}

Nagai et al. reported the first diphenyl ether diarylheptanoid, acerogenin A (23b) [99] and its glycoside (23a) [100] from Acer nickoense and the latter could be enzymatically hydrolyzed to $23 \mathbf{b}$. Later its regioisomer, 24a, was isolated from the same plant and named as acerogenin B (24a) [101]. One of the most impressive characteristics of $\mathbf{2 3 b}$ and $\mathbf{2 4 a}$ is the chemical shift of $\mathrm{H}^{2}$, which resonate at $\delta 5.84$ and 5.46, respectively. Such resonances are highly upfield-shifted compared to the normal chemical resonances of protons $(\delta 7.26)$ on the benzene ring [102] and neighboring $\mathrm{H}^{4}(\delta 6.63$ for $\mathbf{2 3 b}$ and 6.65 for $\mathbf{2 4 a )}$ in the molecule. Such a shift of resonances can be explained by the fact that this proton orients towards the shielding region imposed by the ring current of the neighboring phenyl ring [103]. X-ray crystallographic analysis of the related system such as galeon (26c) [104], maximowicziol (24h) [52], and acerogenin B (24a) [105] has revealed that the main planes of the two phenyls are very close to $90^{\circ}$ to each other.

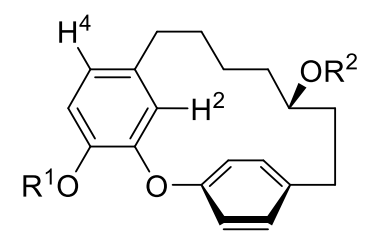

23a (aceroside I, $R^{1}=\beta-D-G I c, R^{2}=H$ )

b (acerogenin $A, R^{1}=R^{2}=H$ )

c [aceroside III, $\mathrm{R}^{1}=\mathrm{H}, \mathrm{R}^{2}=\mathrm{Apif}(1->6) \mathrm{Glc}$ ]

d (aceroside VI, $R^{1}=H, R^{2}=\beta$-D-GIC)

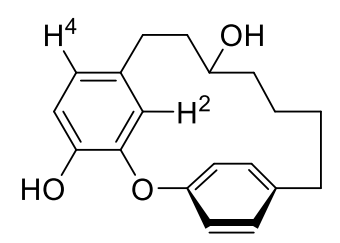

24a (acerogenin B)

Continued studies on the same plants led to the isolation of an additional cyclic diaryl ether heptanoid, aceroside IV (25a) as a glycoside. Hydrolysis of glycoside 25a afforded a new diarylheptanoid, acerogenin C (25b) [106], which was additionally isolated from Boswellia ovalifoliolata [19] later. It should be noted that a regioisomer of $\mathbf{2 5 b}$ was also isolated from Acer nikoense and named as acerogenin L (26a) [64].
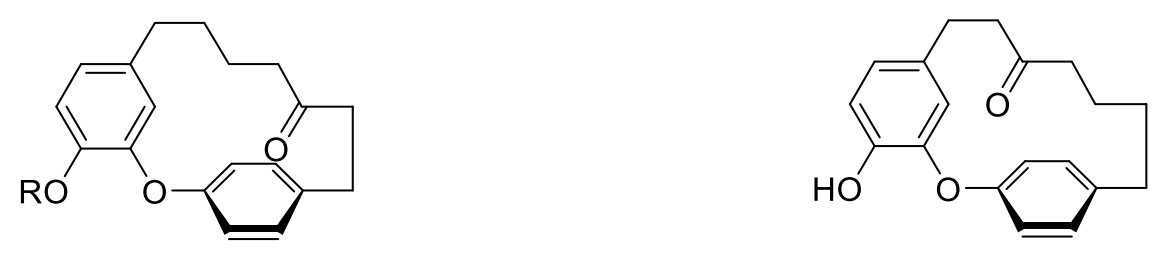

\section{5a (aceroside IV, $R=\beta$-D-Glu) \\ b (acerogenin $\mathrm{C}, \mathrm{R}=\mathrm{H}$ ) enzymatic hydrolysis}

Additional series of cyclic diaryl ether heptanoids, isolated so far, and their biological properties are summarized in Table 5. 
Table 5. The acerogenins with diphenyl ether heptanoid skeleton and their biological properties.

\begin{tabular}{|c|c|c|c|c|c|c|c|c|}
\hline Compound & $\mathrm{R}^{1}$ & $\mathbf{R}^{2}$ & $\mathbf{R}^{3}$ & $X^{1}$ & $X^{2}$ & $X^{3}$ & $X^{4}$ & Reference and Biological Properties \\
\hline acerogenin $\mathrm{A}(\mathbf{2 3 b})$ & $\mathrm{H}$ & $\mathrm{H}$ & $\mathrm{H}$ & $\mathrm{H}, \mathrm{H}$ & $\mathrm{H}, \mathrm{H}$ & $\mathrm{H}, \mathrm{OH}(R)$ & $\mathrm{H}, \mathrm{H}$ & $\begin{array}{c}\text { anti-inflammatory }\left(\mathrm{IC}_{50}=0.32 \mathrm{mg} / \mathrm{ear}\right)[107] \\
\text { osteogenic activity [108] }\end{array}$ \\
\hline acerogenin B (24a) & $\mathrm{H}$ & $\mathrm{H}$ & $\mathrm{H}$ & $\mathrm{H}, \mathrm{H}$ & $\mathrm{H}, \mathrm{OH}$ & $\mathrm{H}, \mathrm{H}$ & $\mathrm{H}, \mathrm{H}$ & $\begin{array}{l}\text { cytotoxicity }\left(\mathrm{IC}_{50}=25.1 \mu \mathrm{M}\right) \text { of }(R) \text {-isomer against } \\
\text { HL60 [109], } \mathrm{Na}^{+} \text {-glucose cotransporter inhibitor [105] }\end{array}$ \\
\hline acerogenin $C(\mathbf{2 5 b})$ & $\mathrm{H}$ & $\mathrm{H}$ & $\mathrm{H}$ & $\mathrm{H}, \mathrm{H}$ & $\mathrm{H}, \mathrm{H}$ & $\mathrm{O}$ & $\mathrm{H}, \mathrm{H}$ & $\begin{array}{l}\text { antibacterial and neuroprotective [110], } \\
\text { NO production inhibitor }\left(\mathrm{IC}_{50}=61.4 \mu \mathrm{M}\right)[111]\end{array}$ \\
\hline acerogenin $\mathrm{D}(\mathbf{2 5 c})$ & $\mathrm{H}$ & $\mathrm{H}$ & $\mathrm{H}$ & $\mathrm{H}, \mathrm{H}$ & $\mathrm{H}, \mathrm{OH}$ & $\mathrm{O}$ & $\mathrm{H}, \mathrm{H}$ & radical scavenging activity $\left(\mathrm{IC}_{50}=40 \mu \mathrm{M}\right)[62]$ \\
\hline acerogenin $F(23 \mathbf{e})^{a}$ & $\mathrm{H}$ & $\mathrm{H}$ & $\mathrm{H}$ & $\mathrm{H}, \mathrm{H}$ & $\mathrm{H}, \mathrm{H}$ & $\mathrm{H}, \mathrm{OH}(R)$ & $\begin{array}{l}\mathrm{H}, \mathrm{OH} \\
\quad(R)\end{array}$ & {$[60]$} \\
\hline acerogenin $\mathrm{H}(\mathbf{2 3 f})$ & $\mathrm{H}$ & $\mathrm{H}$ & $\mathrm{H}$ & $\mathrm{O}$ & $\mathrm{H}, \mathrm{H}$ & $\mathrm{H}, \mathrm{OH}$ & $\mathrm{H}, \mathrm{H}$ & {$[64]$} \\
\hline acerogenin I (24b) & $\mathrm{H}$ & $\mathrm{H}$ & $\mathrm{H}$ & $\mathrm{H}, \mathrm{H}$ & $\mathrm{H}, \mathrm{OH}$ & $\mathrm{H}, \mathrm{H}$ & $\mathrm{H}, \mathrm{OH}$ & [64] \\
\hline acerogenin J (23g) & $\mathrm{H}$ & $\mathrm{H}$ & $\mathrm{H}$ & $\mathrm{H}, \mathrm{H}$ & $\mathrm{H}, \mathrm{H}$ & $\mathrm{H}, \mathrm{OH}(R)$ & $\begin{array}{l}\mathrm{H}, \mathrm{OH} \\
(S)\end{array}$ & {$[64]$} \\
\hline acerogenin L (26a) & $\mathrm{H}$ & $\mathrm{H}$ & $\mathrm{H}$ & $\mathrm{H}, \mathrm{H}$ & $\mathrm{O}$ & $\mathrm{H}, \mathrm{H}$ & $\mathrm{H}, \mathrm{H}$ & [64] \\
\hline acerogenin $\mathrm{M}(\mathbf{2 4 c})$ & $\mathrm{H}$ & $\mathrm{H}$ & $\mathrm{H}$ & $\mathrm{O}$ & $\mathrm{H}, \mathrm{OH}$ & $\mathrm{H}, \mathrm{H}$ & $\mathrm{H}, \mathrm{H}$ & antitumor promoting activity [107] \\
\hline pterocarine $(\mathbf{2 6 b})$ & $\mathrm{H}$ & $\mathrm{OH}$ & $\mathrm{H}$ & $\mathrm{H}, \mathrm{H}$ & $\mathrm{O}$ & $\mathrm{H}, \mathrm{H}$ & $\mathrm{H}, \mathrm{H}$ & cytotoxic against K562 (51\% @100 g/mL) [25] \\
\hline galeon $(\mathbf{2 6 c})$ & $\mathrm{H}$ & $\mathrm{OCH}_{3}$ & $\mathrm{H}$ & $\mathrm{H}, \mathrm{H}$ & $\mathrm{O}$ & $\mathrm{H}, \mathrm{H}$ & $\mathrm{H}, \mathrm{H}$ & [104] \\
\hline 10-hydroxygaleon (26d) & $\mathrm{H}$ & $\mathrm{OCH}_{3}$ & $\mathrm{H}$ & $\mathrm{H}, \mathrm{OH}$ & $\mathrm{O}$ & $\mathrm{H}, \mathrm{H}$ & $\mathrm{H}, \mathrm{H}$ & [104] \\
\hline $24 d$ & $\mathrm{H}$ & $\mathrm{OCH}_{3}$ & $\mathrm{H}$ & $\mathrm{H}, \mathrm{H}$ & $\begin{array}{l}\mathrm{H}, \mathrm{OH} \\
(R)\end{array}$ & $\mathrm{H}, \mathrm{H}$ & $\mathrm{H}, \mathrm{H}$ & [23] \\
\hline myricatomentogenin (26e) & $\mathrm{H}$ & $\mathrm{OCH}_{3}$ & $\mathrm{OH}$ & $\mathrm{H}, \mathrm{H}$ & $\mathrm{O}$ & $\mathrm{H}, \mathrm{H}$ & $\mathrm{H}, \mathrm{H}$ & [112] \\
\hline jugcathanin (juglanin A, 26f) & $\mathrm{CH}_{3}$ & $\mathrm{OCH}_{3}$ & $\mathrm{OH}$ & $\mathrm{H}, \mathrm{H}$ & $\mathrm{O}$ & $\mathrm{H}, \mathrm{H}$ & $\mathrm{H}, \mathrm{H}$ & cytotoxicity $[40,91]$ \\
\hline
\end{tabular}


Table 5. Cont

\begin{tabular}{|c|c|c|c|c|c|c|c|c|}
\hline Compound & $\mathbf{R}^{1}$ & $\mathbf{R}^{2}$ & $\mathbf{R}^{3}$ & $X^{1}$ & $\mathrm{X}^{2}$ & $X^{3}$ & $\mathrm{X}^{4}$ & Reference and Biological Properties \\
\hline platycarynol (24e) & $\mathrm{CH}_{3}$ & $\mathrm{OCH}_{3}$ & $\mathrm{H}$ & $\mathrm{H}, \mathrm{H}$ & $\begin{array}{l}\mathrm{H}, \mathrm{OH} \\
(R)\end{array}$ & $\mathrm{H}, \mathrm{H}$ & $\mathrm{H}, \mathrm{H}$ & $\begin{array}{c}\text { [24], cytotoxic against A549 }\left(\mathrm{IC}_{50}=11.5 \mu \mathrm{g} / \mathrm{mL}\right) \text { [22], } \\
\text { NF-kB inhibitor [113] }\end{array}$ \\
\hline aceroside $B_{1}$ (24fa) & \multicolumn{2}{|c|}{$\beta$-D-GldH } & $\mathrm{H}$ & $\mathrm{H}, \mathrm{H}$ & $\begin{array}{l}\mathrm{H}, \mathrm{OH} \\
(R)\end{array}$ & $\mathrm{H}, \mathrm{H}$ & $\mathrm{H}, \mathrm{H}$ & [61], osteogenic activity [108] \\
\hline aceroside $B_{2}(\mathbf{2 4 f b})$ & \multicolumn{2}{|c|}{$\beta$-D-GldH } & $\mathrm{H}$ & $\mathrm{H}, \mathrm{H}$ & $\begin{array}{l}\mathrm{H}, \mathrm{OH} \\
(S)\end{array}$ & $\mathrm{H}, \mathrm{H}$ & $\mathrm{H}, \mathrm{H}$ & [61] \\
\hline aceroside I (23h) & \multicolumn{2}{|c|}{$\beta$-D-GldH } & $\mathrm{H}$ & $\mathrm{H}, \mathrm{H}$ & $\mathrm{H}, \mathrm{H}$ & $\mathrm{H}, \mathrm{OH}(R)$ & $\mathrm{H}, \mathrm{H}$ & [114], osteogenic activity [108] \\
\hline aceroside II (24g) & $\mathrm{H}$ & $\mathrm{H}$ & $\mathrm{H}$ & $\mathrm{H}, \mathrm{H}$ & $\begin{array}{c}\mathrm{H}, \\
\text { O- } \beta-\mathrm{D}-\mathrm{Glc}\end{array}$ & $\mathrm{H}, \mathrm{H}$ & $\mathrm{H}, \mathrm{H}$ & 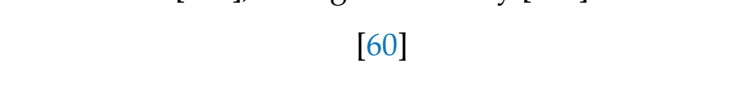 \\
\hline aceroside III (23i) & $\mathrm{H}$ & $\mathrm{H}$ & $\mathrm{H}$ & $\mathrm{H}, \mathrm{H}$ & $\mathrm{H}, \mathrm{H}$ & $\mathrm{H}, \mathrm{Y}^{\mathrm{c}}$ & $\mathrm{H}, \mathrm{H}$ & $\begin{array}{c}\text { radical scavenging activity }\left(\mathrm{IC}_{50}=40 \mu \mathrm{M}\right) \text {, osteogenic } \\
\text { activity [108] }\end{array}$ \\
\hline aceroside IV (25a) & \multicolumn{2}{|c|}{$\beta$-D-GldH } & $\mathrm{H}$ & $\mathrm{H}, \mathrm{H}$ & $\mathrm{H}, \mathrm{H}$ & $\mathrm{O}$ & $\mathrm{H}, \mathrm{H}$ & [106] \\
\hline aceroside V (25d) & \multicolumn{2}{|c|}{$\beta$-D-GldH } & $\mathrm{H}$ & $\mathrm{H}, \mathrm{H}$ & $\mathrm{H}, \mathrm{OH}$ & $\mathrm{O}$ & $\mathrm{H}, \mathrm{H}$ & [62] \\
\hline aceroside $\mathrm{VI}^{\mathrm{b}}(\mathbf{2} \mathbf{j} \mathbf{j})$ & $\mathrm{H}$ & $\mathrm{H}$ & $\mathrm{H}$ & $\mathrm{H}, \mathrm{H}$ & $\mathrm{H}, \mathrm{H}$ & $\mathrm{H}, \mathrm{O}-\beta$-D-Glc & $\mathrm{H}, \mathrm{H}$ & [115] \\
\hline 9-oxoacerogenin A (23k) & $\mathrm{H}$ & $\mathrm{H}$ & $\mathrm{H}$ & $\mathrm{H}, \mathrm{H}$ & $\mathrm{O}$ & $\mathrm{H}, \mathrm{OH}$ & $\mathrm{H}, \mathrm{H}$ & anti-melanogenesis (17.6\% @100 $\mu \mathrm{M})$ [109] \\
\hline maximowicziol A (24h) & $\mathrm{H}$ & $\mathrm{H}$ & $\mathrm{H}$ & $\mathrm{H}, \mathrm{H}$ & $\begin{array}{l}\mathrm{H}, \mathrm{OH} \\
(S)\end{array}$ & $\mathrm{H}, \mathrm{H}$ & $\begin{array}{l}\mathrm{H}, \mathrm{OH} \\
(S)\end{array}$ & [52] \\
\hline $24 \mathbf{i}$ & $\mathrm{CH}_{3}$ & \multicolumn{2}{|c|}{$\mathrm{OCH}_{3} \mathrm{OH}$} & $\mathrm{H}, \mathrm{H}$ & $\mathrm{H}, \mathrm{OH}$ & $\mathrm{H}, \mathrm{H}$ & $\mathrm{H}, \mathrm{H}$ & cytotoxic against $\mathrm{A} 549\left(\mathrm{IC}_{50}=11.5 \mu \mathrm{g} / \mathrm{mL}\right)[22]$ \\
\hline jugsigin A (24j) & $\mathrm{H}$ & $\mathrm{H}$ & $\mathrm{OH}$ & $\mathrm{H}, \mathrm{H}$ & $\begin{array}{l}\mathrm{H}, \mathrm{OH} \\
(S)\end{array}$ & $\mathrm{H}, \mathrm{H}$ & $\mathrm{H}, \mathrm{H}$ & cytotoxic against HT-29 [116] \\
\hline 2-methylacerogenin A (231) & $\mathrm{CH}_{3}$ & $\mathrm{H}$ & $\mathrm{H}$ & $\mathrm{H}, \mathrm{H}$ & $\mathrm{H}, \mathrm{H}$ & $\mathrm{H}, \mathrm{OH}(S)$ & $\mathrm{H}, \mathrm{H}$ & [100] \\
\hline
\end{tabular}


To determine the geometries of acerogenins, the splitting pattern of the 4 protons $\left(\mathrm{H}^{2}, \mathrm{H}^{3}, \mathrm{H}^{5}\right.$ and $\mathrm{H}^{6}$ ) on para-disubstituted phenyl rings of acerogenins A (23b), C (25b), B (24a) and L (26a) in their ${ }^{1} \mathrm{H}$ NMR spectra may afford important information. Only two resonances were found for these protons appearing as an $\mathrm{AB}$ quartet $\left({ }^{3} \mathrm{~J}=8.3 \mathrm{~Hz}\right)$ in the spectra of acerogenins $\mathrm{C}$ and $\mathrm{L}$, while four resonances, each being a doublet of doublet, were observed for the those of acerogenin $\mathrm{A}$ and $\mathrm{B}$, respectively. These results may indicate that two sets of protons on the para-substituted phenyls in acerogenins $\mathrm{A}$ and $\mathrm{B}$, such as $\mathrm{H}^{2}$ and $\mathrm{H}^{6}, \mathrm{H}^{3}$ and $\mathrm{H}^{5}$, are not equivalents, and may indicate a possible difference in the rotational energy barrier around the diaryl ether bond, unlike those in acerogenins $\mathrm{C}$ and $\mathrm{L}$. The energy barriers in acerogenins $\mathrm{C}$ and $\mathrm{L}$ are, therefore, somewhat lower than those of acerogenins A and B, respectively. Indeed, the minimized energies of $25 \mathbf{b}(2.65 \mathrm{Kcal} / \mathrm{mole})$ and $26 \mathbf{a}$ $(3.85 \mathrm{Kcal} / \mathrm{mole})$ calculated by Chem3D Pro ${ }^{\circledR}$ are much lower than those of $23 \mathbf{b}(10.11 \mathrm{Kcal} / \mathrm{mole})$ and 24a $(10.15 \mathrm{Kcal} / \mathrm{mole})$. The presence of $s p^{2}$-hybridized carbon (carbonyl) in the heptyl chain of $\mathbf{2 5 b}$ and 26a may reduce both angle strain and $\mathrm{H}-\mathrm{H}$ steric interactions relative to those of $\mathbf{2 3 b}$ and 24a, respectively. Such a rotational energy barrier in the systems with a substituent such as galeon would be increased sufficiently enough not to rotate, leading to two atropisomers. Indeed, galeon has been isolated on two separate occasions in both levo- [104] and dextrorotatory [112] ones from Myrica gale, respectively. The configuration of planar chirality was determined by X-ray crystallographic analysis of the corresponding 4-bromobenzoate (26d) of (-)-galeon to be $M-(-)$-galeon $(\mathbf{2 6} \mathbf{c b})$ and thus dextrorotatory one was determined as $P-(+)$-galeon $(\mathbf{2 6} \mathbf{c a})$. It should be noted that the nature of conformational chirality, the racemization mechanisms, and predictions of these for diaryl ether heptanoids have been recently studied [119].

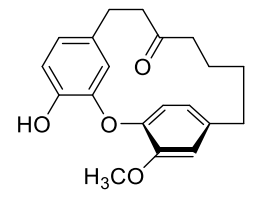

26ca $[P-(+)$-galeon]

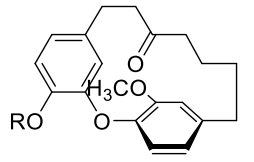

26cb $[M-(-)$-galeon, $\mathrm{R}=\mathrm{H}]$ d $(\mathrm{R}=4$-bromobenzoyl)

After we isolated galeon (26) [22] and its derivatives 27a,b,c [23] from the roots of Juglans mandshirica, and a series of related diaryl ether heptanoids, 27d-f from the stem of Engelhardia roxburghiana [21,120], were additionally isolated. Unfortunately, the plane chirality was not defined in all cases and remained to be clarified. The originally proposed structure 28 [120] as engelhardione was later revised as to $\mathbf{2 7 f}$ [21], which is identical to previously reported pterocarine (26b) [25] and revised structure was confirmed by total synthesis (vide infra). The compound 27c showed strong inhibitory activity $(95.7 \%)$ on topoisomerase II at $50 \mu \mathrm{g} / \mathrm{mL}$ level [121]. Engelhardiols A (27d) and B (27e) displayed anti-tubercular activity with MIC values of 72.7 and $62.1 \mu \mathrm{M}$, respectively [21]. Related engelhardione has MIC of $0.2 \mu \mathrm{g} / \mathrm{mL}$ against Mycobacterium tuberculosis (H37Rv) [120].

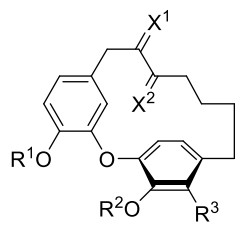

27a $\left[R^{1}=\mathrm{R}^{2}=\mathrm{CH}_{3}, \mathrm{R}^{3}=\mathrm{OH}, \mathrm{X}^{1}=\mathrm{H}, \mathrm{OH}, \mathrm{X}^{2}=\mathrm{H}, \mathrm{H}\right]$ b $\left[\mathrm{R}^{1}=\mathrm{R}^{3}=\mathrm{H}, \mathrm{R}^{2}=\mathrm{CH}_{3}, \mathrm{X}^{1}=\mathrm{H}, \mathrm{OH}(R), \mathrm{X}^{2}=\mathrm{O}\right]$ c $\left[\mathrm{R}^{1}=\mathrm{R}^{3}=\mathrm{H}, \mathrm{R}^{2}=\mathrm{CH}_{3}, \mathrm{X}^{1}=\mathrm{H}, \mathrm{H}, \mathrm{X}^{2}=\mathrm{H}, \mathrm{OH}(R)\right]$ d [engelhardiol $\mathrm{A}, \mathrm{R}^{1}=\mathrm{R}^{3}=\mathrm{H}, \mathrm{R}^{2}=\mathrm{CH}_{3}, \mathrm{X}^{1}=\mathrm{H}, \mathrm{OH}(R), \mathrm{X}^{2}=\mathrm{H}, \mathrm{OH}(S)$ ] e [engelhardiol $B, R^{1}=R^{3}=H, R^{2}=C_{3}, X^{1}=H, O H(S), X^{2}=H, O H(S)$ ] f (engelhardione, $\left.\mathrm{R}^{1}=\mathrm{R}^{2}=\mathrm{R}^{3}=\mathrm{H}, \mathrm{X}^{1}=\mathrm{H}, \mathrm{H}, \mathrm{X}^{2}=\mathrm{O}\right]$<smiles>O=C(O)CCCCc1ccc(O)c(Oc2cc(CCCCC(=O)CO)ccc2O)c1</smiles>

28 


\subsubsection{Garuganins and Garugamblins}

A series of cyclic diarylheptanoids with a double bond in a heptyl skeleton were isolated such as 29a (garugamblin-1) and 31 (garugamblin-2) from Garuga gamblei [122,123], garugamblin-3 from Alnus japonica [124], and 29b (garuganin I) [125,126], 30a (garuganin III) [127], 30b (garuganin IV), 32 (garuganin VI) [17], and related garugamblins such as 29c and 30c [18] from Garuga pinata. Although the structures of garugamblin-1 (29a), garugamblin-2 (31) [123] and garuganin I (29b) [125] were confirmed by $\mathrm{X}$-ray structure analysis, the originally proposed structures of garuganin III (30a), garuganin IV (30b), and 1,9'-didesmethylgaruganin III (30c) were revised later through total synthesis to be 33, 29a, and 30d (garuganin VII), respectively [128,129].
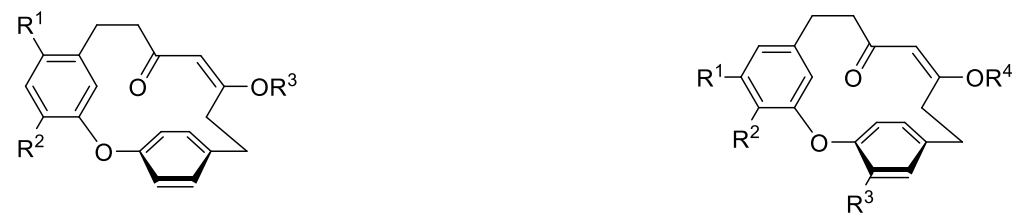

$$
\begin{aligned}
& \text { 29a (garugamblin } 1, R^{1}=H, R^{2}=O C_{3}, R^{3}=C_{3} \text { ) 30a (garuganin III, } R^{1}=R^{2}=O C H_{3}, R^{3}=H, R^{4}=C_{3} \text { ) } \\
& \text { b (garuganin I, } \mathrm{R}^{1}=\mathrm{R}^{2}=\mathrm{OCH}_{3}, \mathrm{R}^{3}=\mathrm{CH}_{3} \text { ) } \\
& \text { c (9'-desmethylgarugamblin } 1, \mathrm{R}^{1}=\mathrm{R}^{3}=\mathrm{H} \\
& \left.\mathrm{R}^{2}=\mathrm{OCH}_{3}\right) \\
& \text { d (garugamblin } 3, R^{1}=R^{2}=R^{3}=H \text { ) } \\
& =>\text { revised to } \mathbf{3 3} \\
& \text { b (garuganin IV, } \mathrm{R}^{1}=\mathrm{OCH}_{3}, \mathrm{R}^{2}=\mathrm{R}^{3}=\mathrm{H}, \mathrm{R}^{4}=\mathrm{CH}_{3} \text { ) } \\
& =>\text { revised to } 29 \mathrm{a}
\end{aligned}
$$

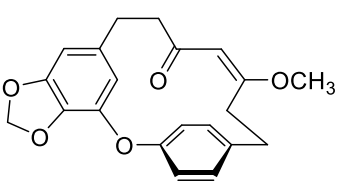

31 (garugamblin 2)

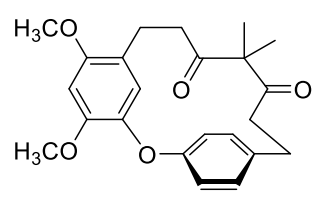

32 (garuganin $\mathrm{VI}$ )

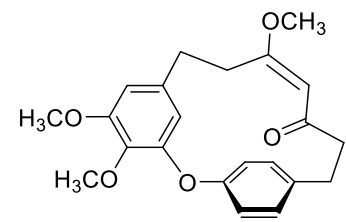

33

It should be noted that recent dynamic NMR studies revealed that garuganin I can undergo conformational enantiomerism, leading to racemization through the rotation of the $C^{7}-C^{8}$ and $C^{12}-C^{13}$ sigma bonds [130].

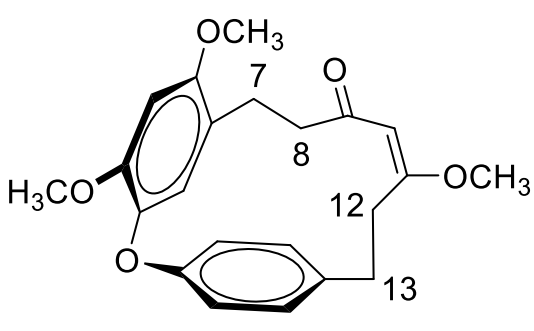

garuganin I (29b)

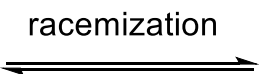

ent-garuganin I (ent-29b)

\subsubsection{Miscellaneous}

In addition, a very potent $\left(\mathrm{IC}_{50}=3.9 \mu \mathrm{g} / \mathrm{mL}\right)$ leishmanicidal [59] diaryl heptenoid, $\mathbf{3 4}$ from Betula platyphylla [65], B. davurica [66], and B. ovalifolia [131], and ovalifoliolatin (35) from Boswellia ovalifoliolata [19] were reported as additional diaryl ether heptenoids. The cyclic diaryl ether heptenoids $\mathbf{3 5}$ are unusual trans-cycloalkenes isolated from the nature and showed potent antibacterial activity against Staphylococcus aureus and Chromobacterium violaceum. 


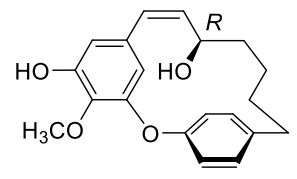

34

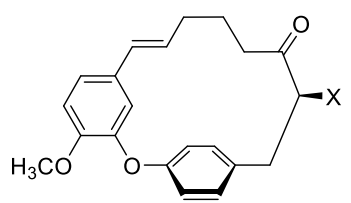

35a [ovalifoliolatin $\mathrm{A}, \mathrm{X}=\mathrm{OH}(S)$ ]

b (ovalifoliolatin $B, X=H$ )

An additional series of diaryl ether heptenoids, giffonins A-K, Q, R, and S, were isolated from the leaves of the hazelnut tree (Corylus avellana) $[11,12,132]$ which are summarized in Table 6. Diaryl ether heptenoids, giffonin $\mathrm{D}$ and giffonin $\mathrm{H}$ at $10 \mu \mathrm{M}$ reduced both $\mathrm{H}_{2} \mathrm{O}_{2}{ }^{-}$and $\mathrm{H}_{2} \mathrm{O}_{2} / \mathrm{Fe}^{2+}$-induced lipid peroxidation by more than $60 \%$ and $50 \%$, respectively, being more active than curcumin $(19.2 \%)$. When hazelnuts were infected by so-called "Cimiciato", the composition of the constituents was changed to produce a new oxa[7.1]metametacyclophane, 40, along with asadanin (1a) and giffonin P (7f) [133].

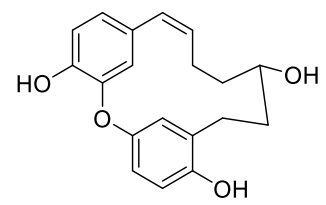

40

Table 6. Diaryl ether heptenoids, giffonin A-K, Q, R, and S.

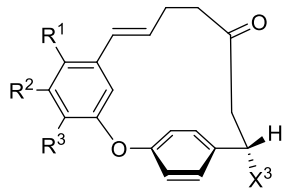

36

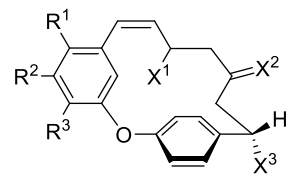

37

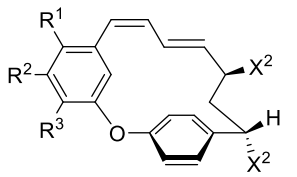

38

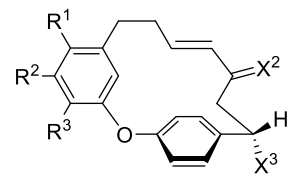

39

\begin{tabular}{lccccccc}
\hline & $\mathbf{R}^{\mathbf{1}}$ & $\mathbf{R}^{\mathbf{2}}$ & $\mathbf{R}^{\mathbf{3}}$ & $\mathbf{X}^{\mathbf{1}}$ & $\mathbf{X}^{\mathbf{2}}$ & $\mathbf{X}^{\mathbf{3}}$ & Reference \\
\hline giffonin A (36a) & $\mathrm{OCH}_{3}$ & $\mathrm{OH}$ & $\mathrm{OCH}_{3}$ & - & - & $\mathrm{H}$ & {$[11]$} \\
giffonin B (36b) & $\mathrm{OCH}_{3}$ & $\mathrm{OH}$ & $\mathrm{OCH}_{3}$ & - & - & $\mathrm{OH}(S)$ & {$[11]$} \\
giffonin C (37a) & $\mathrm{OCH}_{3}$ & $\mathrm{OH}$ & $\mathrm{OCH}_{3}$ & $\mathrm{H}$ & $\mathrm{H}, \mathrm{OH}(R)$ & $\mathrm{H}$ & {$[11]$} \\
giffonin D (37b) & $\mathrm{OCH}_{3}$ & $\mathrm{OH}$ & $\mathrm{OCH}_{3}$ & $\mathrm{H}$ & $\mathrm{O}$ & $\mathrm{H}$ & {$[11]$} \\
giffonin E (37c) & $\mathrm{OCH}_{3}$ & $\mathrm{OH}$ & $\mathrm{OCH}_{3}$ & $\mathrm{OH}(R)$ & $\mathrm{H}, \mathrm{H}$ & $\mathrm{H}$ & {$[11]$} \\
giffonin F (37d) & $\mathrm{OCH}_{3}$ & $\mathrm{OH}$ & $\mathrm{OCH}_{3}$ & $\mathrm{OH}(R)$ & $\mathrm{O}$ & $\mathrm{H}$ & {$[11]$} \\
giffonin G (38a) & $\mathrm{OCH}_{3}$ & $\mathrm{OH}$ & $\mathrm{OCH}_{3}$ & - & $\mathrm{H}, \mathrm{OH}(S)$ & $\mathrm{H}$ & {$[11]$} \\
giffonin H (38b) & $\mathrm{H}$ & $\mathrm{OH}$ & $\mathrm{OCH}_{3}$ & - & $\mathrm{H}, \mathrm{OH}(S)$ & $\mathrm{H}$ & {$[11]$} \\
giffonin J a (37e) & $\mathrm{OCH}_{3}$ & $\mathrm{OH}$ & $\mathrm{OCH}_{3}$ & $\mathrm{H}$ & $\mathrm{H}, \mathrm{OH}(R)$ & $\mathrm{OH}(S)$ & {$[12]$} \\
giffonin K (38c) & $\mathrm{OCH}_{3}$ & $\mathrm{OH}$ & $\mathrm{OCH}$ & - & $\mathrm{H}, \mathrm{OH}(S)$ & $\mathrm{OH}(S)$ & {$[12]$} \\
giffonin Q (39) & $\mathrm{H}$ & $\mathrm{H}$ & $\mathrm{H}$ & - & $\mathrm{O}$ & $\mathrm{H}$ & {$[132]$} \\
giffonin R (38d) & $\mathrm{H}$ & $\mathrm{H}$ & $\mathrm{OH}$ & - & $\mathrm{O}$ & $\mathrm{H}$ & {$[132]$} \\
giffonin S (38e) & $\mathrm{H}$ & $\mathrm{OCH}$ & $\mathrm{OH}$ & - & $\mathrm{O}$ & $\mathrm{H}$ & {$[132]$} \\
\hline & $\mathrm{O}$ & & & & & &
\end{tabular}

a Giffonin I, L-P, T, and U are biphenyl heptanoids (vide supra).

A unique cyclic diaryl heptadienoid tedarene A (41a) was isolated from the marine sponge Tedania ignis [42] and exhibited a couple of intriguing features. Although two double bonds can impose strain onto the cyclic heptanoid ring in tedarene A, the energy barrier between the two atropisomers is not sufficiently high to prevent interconversion at room temperature showing only one set of ${ }^{1} \mathrm{H}$ resonances in NMR spectrum without any of protons on para-substituted phenyl ring. Such a phenomenon reflects that the interconversion rate between the two atropisomers is, coincidently, in the coalescence region at room temperature to cause the proton resonances of $\mathrm{H}^{2}$ and $\mathrm{H}^{6}$, as well as $\mathrm{H}^{3}$ and $\mathrm{H}^{5}$, of the para-substituted phenyl ring to be sufficiently broadened to be undetectable. The rotational energy barrier of 41a was calculated by molecular dynamics simulation to be $14.0 \mathrm{Kcal} / \mathrm{mol}$, which 
is way below the energy barrier $(23.3 \mathrm{Kcal} / \mathrm{mol})$ to define atropisomers [134,135]. A diphenyl ether heptatrienoid $\mathbf{4 2}$ was also isolated from Ostryopsis nobilis (Betulaceae) and the structure was confirmed by X-ray crystal structure analysis [16].

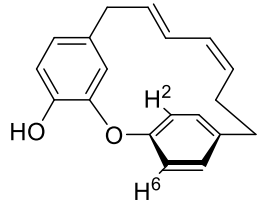

41a (tendarene $A$ )

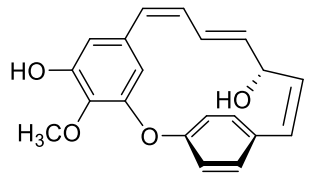

42 (ostryopsitrienol)

\section{Total Synthesis of Biphenyl Diarylheptanoids}

\subsection{Biosynthetic Pathway}

Before the total synthesis of cyclic diarylheptanoids was considered, studies on the biosynthetic pathways are addressed. Elegant studies on the degradation of acerogenin A and the radioisotope-tracing biosynthetic pathway for 1,7-diaylheptanoids revealed that two cinnamate units coupled to a malonate via acetyl CoA leading to the 1,7-diphenylheptanoid, curcumin [136,137], which was further converted to centrolobol. In addition, Fujita and his coworkers performed two radioisotope experiments [138]: They fed labelled compounds, DL-[3- $\left.{ }^{14} \mathrm{C}\right]$ phenylalanine and $\left[3-{ }^{14} \mathrm{C}\right] \mathrm{cinnamic}$ acid to the young shoots of Acer nikoense and found that $95 \%$ of the radioactivity of acerogenin remained on the $\mathrm{C} 1 / \mathrm{C} 7$, but in the case of $\mathrm{L}-1-{ }^{13} \mathrm{C}$ ]phenylalanine, only $5 \%$ of the radioactivity was incorporated at the positions $\mathrm{C} 1 / \mathrm{C} 7$ in acerogenin $\mathrm{A}$. On the other hand, feeding $\left[2-{ }^{14} \mathrm{C}\right]$ sodium acetate, $\left[2-{ }^{14} \mathrm{C}\right]$ malonic acid, and $\left[1-{ }^{14} \mathrm{C}\right]$ sodium acetate resulted in the sufficient incorporation of $\left[2-{ }^{14} \mathrm{C}\right]$ sodium acetate and $\left[2-{ }^{14} \mathrm{C}\right]$ malonic acid into the acerogenin, but not $\left[1-{ }^{14} \mathrm{C}\right]$ sodium acetate. These observations indicate the possible biosynthetic pathway of acerogenin A (Scheme 1), via an intramolecular oxidative coupling of its linear precursor, centrolobol (43).<smiles>O=C(O)C=Cc1ccc(O)cc1</smiles>

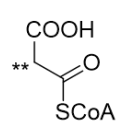<smiles>CC#CC</smiles><smiles>CC(CCCC(O)Cc1ccc(O)cc1)Cc1ccc(O)cc1</smiles>

centrolobol (43)

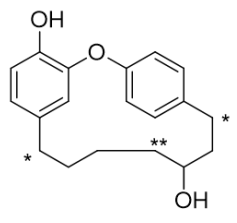

acerogenin $\mathrm{A}(\mathbf{2 3 b})$

Scheme 1. Propsed biosynthetic pathway for the linear and cyclic diaryl ether heptanoids.

It should be noted that Watanabe et al. isolated alnusonol (5a) along with its suspected precursor platyphyllonol (44) [53], from which one may deduce the presence of dicarbon radical as a possible intermediate in the biosynthetic pathway for the (9S)-alnusonol (5a) via C-C coupling.

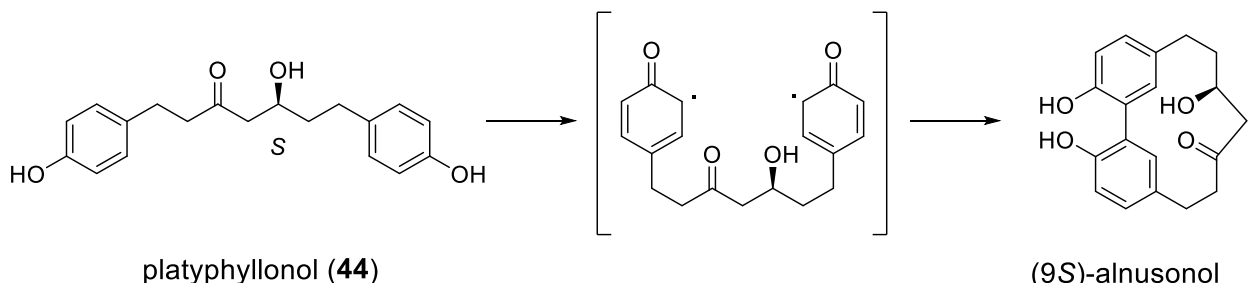


Such a premise was proved by the isolation of three cyclized diarylheptanoids such as acerogenin A (23b), (R)-acerogenin B (24a), and acerogenin E (51), and a linear diarylheptanoid, $(-)$-centrolobol together from the same plants indicates that the former two are biosynthetically related to (-)-centrolobol [107]. In addition, many plants have afforded various combinations of two from the three diarylheptanoids (i.e., linear, biphenyl heptanoids and diphenyl ether heptanoids). One possible explanation for these results comes from the intramolecular oxidative coupling of phenolic linear diarylheptanoid via the free diradicals: A phenolic oxidative $\mathrm{C}-\mathrm{C}$ coupling would lead to meta,meta-bridged biphenyl heptanoids, whereas the corresponding $\mathrm{C}-\mathrm{O}$ coupling would result in the alternative, meta,para-bridged diphenyl ether heptanoids.

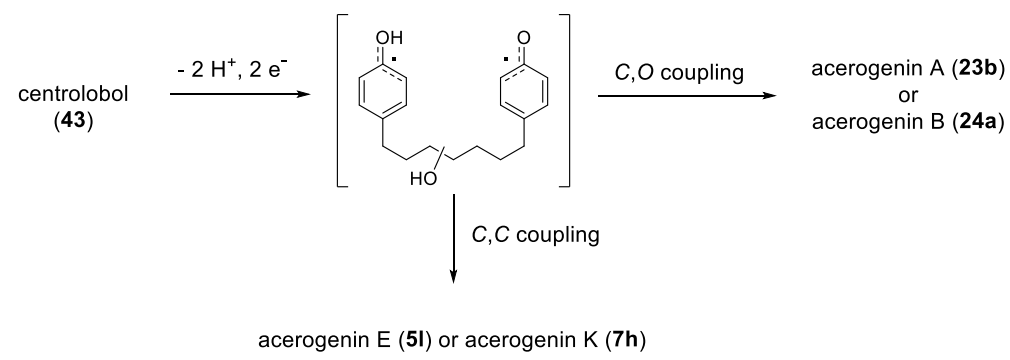

\subsection{Total Synthesis of Biphenyl Heptanoids}

The formation of the aryl-aryl bond has long been of interest in the development of facile synthetic methods, i.e., metal catalyzed coupling reactions, such as Suzuki [139], Stille [140], Negishi [141], Ullmann [142,143], and electro- as well as photochemical methods [144]. Among these, the Suzuki reaction, the Ullmann reaction, and the photochemical process have been applied to biphenyl diarylheptanoids at the final step. The Wittig [145], Thorpe [146], and olefin metathesis [147] reactions have also been employed for the $\mathrm{C}-\mathrm{C}$ bond formation in the heptane moiety. However, attempts using Thorpe reaction failed to afford the desired cyclic biphenyl heptanoids [148], which would, thus, not be discussed herein.

\subsubsection{Aryl-Aryl Bond Formation via Metal Catalyzed Coupling}

The Ni(0)-promoted intramolecular coupling of aryl halide developed by Semmelhack [149] was applied to the total synthesis upon biphenyl heptanoid, alnusone (4a) for the first time [150]. The reaction of diiodide 49 in the presence of tetrakis(triphenylphosphine)nickel afforded methyl-protected alnusone (50) in $46 \%$ yield. The acid-catalyzed deprotection of methyl group produced $4 \mathbf{a}$ with $72 \%$ yield.
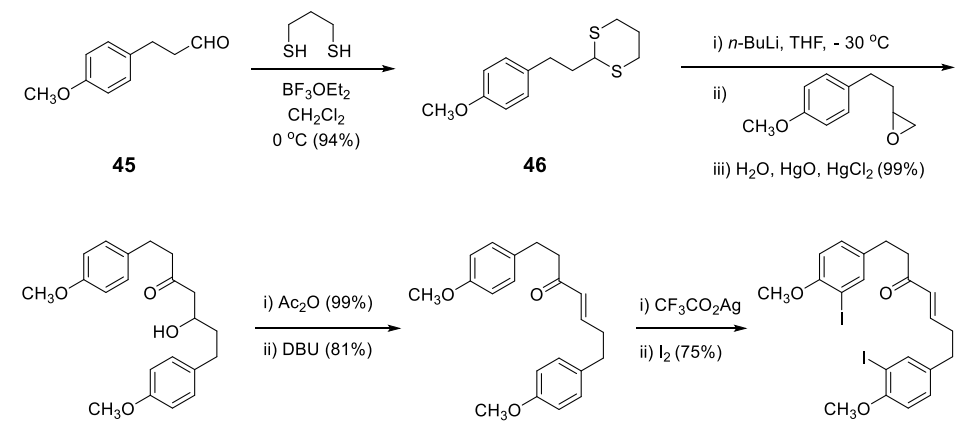

47
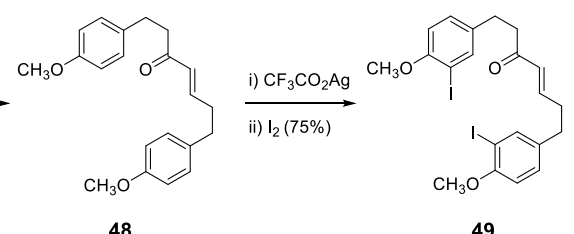

48

49

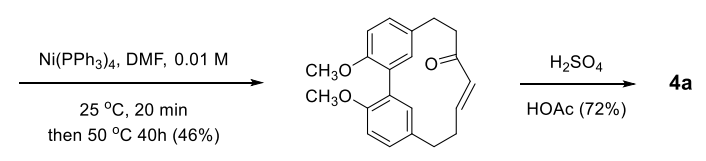


The starting compound 49 was prepared from 3-(4-methoxyphenyl)propanal (45) via thioacetal formation with propane-1,3-dithiol, lithiation by $n$-BuLi, a reaction with 2-(4-methoxyphenethyl)oxirane, dehydration by DBU, and iodination.

The same strategy has been applied to the synthesis of myricanone and myricanol by Whiting and Wood [151]. The intramolecular ring closure of diiodides $\mathbf{5 4}$ provided the corresponding benzyl-protected myricanone (55a) and myricanol (55b) in 10\% and 7.3\% yield, respectively. The low yield was explained by angle strain and van der Waals hydrogen interaction caused by the $s p^{3}$-hybridized methylene units compared to those of $s p^{2}$-hybridized $E$-methine in the former molecule, alnusone. The subsequent hydrogenolysis of 55a afforded 11a while 12a was prepared by hydrogenolysis of $\mathbf{5 5 b}$ followed by hydrolysis. It should be noted that authors did not mention about either the presence of stereoisomers or NMR spectral data for the compound $\mathbf{5 5 b}$ even though $\mathbf{5 5 b}$ has both a chirality center and a chiral axis. The starting $54 \mathbf{a}, \mathbf{b}$ were prepared by iodination of 53a,c, respectively. The Grignard reagent generated from 51 was reacted with 3-(4-benzyloxyphenyl)propanal (52) to provide 53a in $21 \%$ yield. Acetylation of 53a gave $53 \mathbf{b}$ and oxidation of 53a with pyridinium chlorochromate (PCC) gave 53c [152].<smiles>[B]CCCCc1ccc(OC)c(OC)c1OCc1ccccc1</smiles>

51<smiles>[X]C(CCCCc1ccc(OC)c(OC)c1OCc1ccccc1)CCc1ccc(OCc2ccccc2)cc1</smiles><smiles>[X]C(CCCCc1cc(I)c(OC)c(OC)c1OCc1ccccc1)CCc1ccc(OCc2ccccc2)c(I)c1</smiles>

$$
\begin{aligned}
54 a(X & =O) \text { from } 53 c \\
\mathbf{b}(X & =H, \text { OAc }) \text { from 53b }
\end{aligned}
$$

52

52

$$
\text { PCC } \begin{aligned}
& \text { 53a }(X=\mathrm{H}, \mathrm{OH})(21 \%) \\
& \mathbf{b}(\mathrm{X}=\mathrm{H}, \mathrm{OAc}) \\
& \quad \mathbf{c}(\mathrm{X}=\mathrm{O})
\end{aligned}
$$
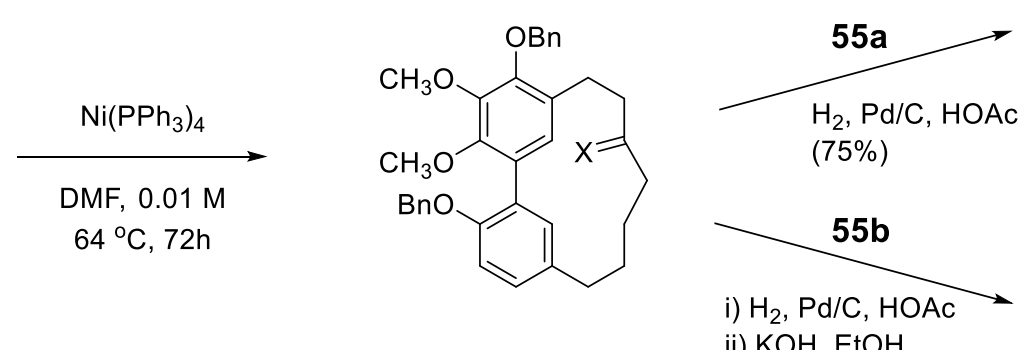

$11 \mathrm{a}$

$$
\begin{aligned}
55 \mathbf{a}(X=O)(10 \%) \\
\mathbf{b}(X=H, O A c)(7.3 \%)
\end{aligned}
$$

Ogura and Usuki employed the same strategy to prepare acerogenin E (5l) and K (7h) [153], in which the $\mathrm{Ni}(0)$-catalyzed reaction was modified by a more recent methodology of a domino sequence of a Miyaura arylborylation and an intramolecular Suzuki reaction [154] of 59 to dimethylacerogenin $\mathrm{E}(\mathbf{6 0})$, of which deprotection by $\mathrm{BBr}_{3}$ to yield acerogenin $\mathrm{E}$ (51). The reduction of 60 with $\mathrm{NaBH}_{4}$ was followed by demethylation with $\mathrm{BBr}_{3}$ to provide acerogenin $\mathrm{K}$ (7h) in $95 \%$ yield. The starting 59 was prepared from 56 via Claisen-Schmidt condensation, catalytic reduction, and $\mathrm{CF}_{3} \mathrm{CO}_{2} \mathrm{Ag}$ mediated iodination. 


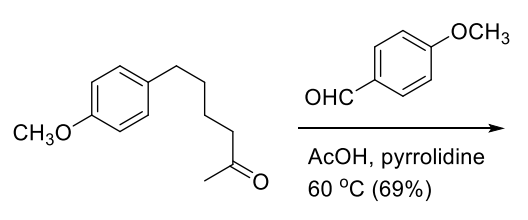

56

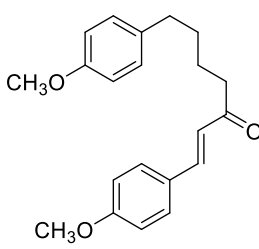

57

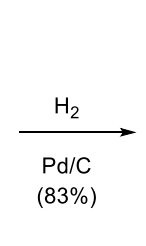

$83 \%)$

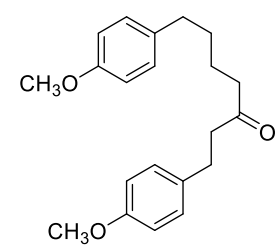

58<smiles>COc1ccc(CCCCC(=O)CCc2ccc(OC)c(I)c2)cc1I</smiles>

59

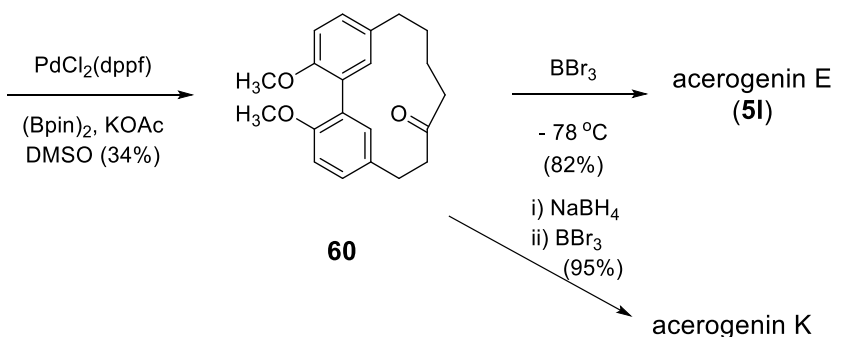

(7h)

The similar oxidative homocoupling of the diboronic ester (62) also gave 60 in 50\% yield [155]. The starting $\mathbf{6 2}$ was prepared from 58 via glycol protection of ketone, reaction with $\mathrm{Cr}(\mathrm{CO})_{6}$, borylation with isopropoxy-4,4-5,5-tetramethyl-1,3,2-dioxoborane, and the deprotection of ketone.

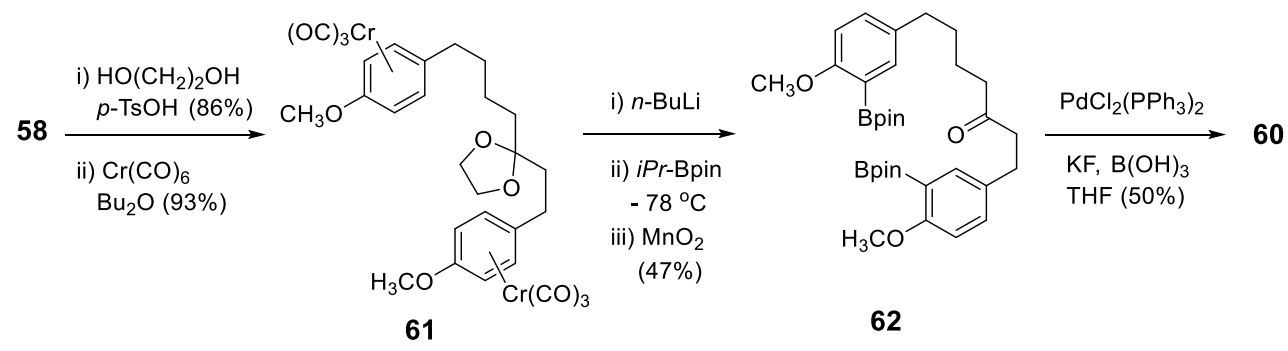

It should be noted that neither the reduction of the double bond in 63 to 64 nor the direct cyclization of $\mathbf{6 3}$ to $\mathbf{6 5}$ under Suzuki-Miyaura coupling was successful [153]; thus, the cyclization of $\mathbf{6 4}$ using the above reaction conditions has not been pursued.

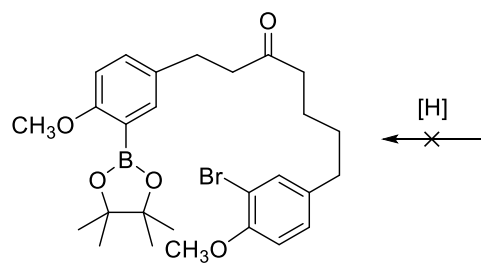

64<smiles>COc1ccc(/C=C/C(=O)CCCCc2ccc(O)c(Br)c2)cc1B1OC(C)(C)C(C)(C)O1</smiles>

63
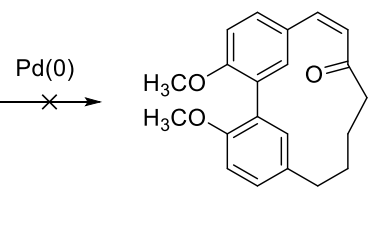

65

Such a method was revisited by Martin et al. for the synthesis of myricanol [71]. The intramolecular cross Suzuki-Miyaura coupling of compound 67, possessing an arylboronic acid pinacol ester and an aryl iodide resulted in a benzyl protected compound 68 as a racemic mixture. Debenzylation by hydrogenolysis, followed by enantioselective reduction of the keto group using K-selectride, led to a racemic mixture of 12aa, which was then resolved by chiral high-performance liquid chromatography (HPLC) to afford (+)-12aa- $(\mathrm{a} R)(11 S)$ and $(-)-\mathbf{1 2} \mathbf{a a} \mathbf{a}^{-}-(\mathrm{a} S)(11 R)$, of which the absolute configuration were determined by $X$-ray analysis. The starting compound 67 was prepared by Claisen-Schmidt reaction of 65 and 66 in $39 \%$ yield. 


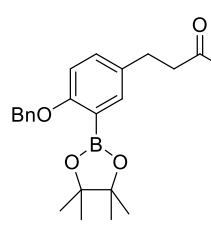

66

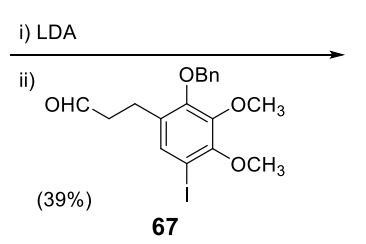<smiles>COc1c(I)cc(CC/C=C/C(=O)CCc2ccc(OCc3ccccc3)c(B3OC(C)(C)C(C)(C)O3)c2)c(OCc2ccccc2)c1OC</smiles>

68

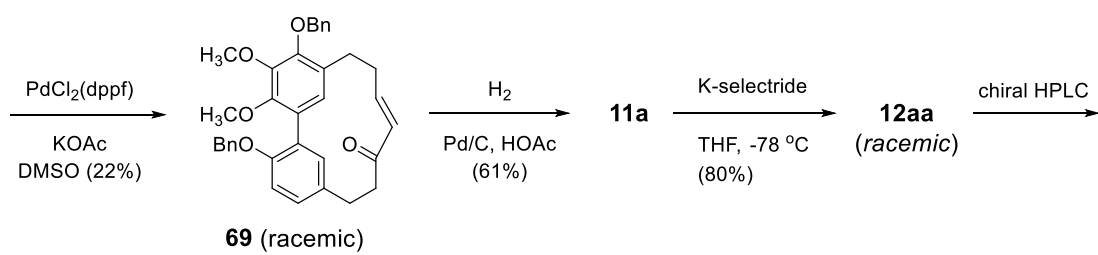<smiles>COc1c(O)cccc1CCCCC(O)CCc1ccc(O)c(OC)c1O</smiles>

(+)-12aa $(\mathrm{a} R)(S)$

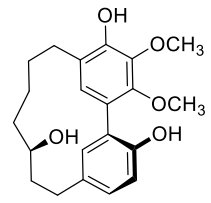

$(-)-12 a^{\prime}(\mathrm{a} S)(R)$

The emerging interest in (-)-aS, $11 R$-myricanol owing to its ability to lower the tau protein level led to a couple of the enantioselective total synthesis: one covers a synthesis of the biaryl macrocycle skeleton via Suzuki-Miyaura cross coupling and ring-closing metathesis reactions [156], and the other includes an enantioselective asymmetric Suzuki cross-coupling, and an indium-mediated allylation of an aliphatic aldehyde [157]. Unfortunately, detailed procedures for these methods are currently unavailable. However, the successful enantioselective Suzuki-Miyaura cross coupling for synthesis of subclass linear diarylheptanoid, diospongin B [158], may open a vista to the enantioselective synthesis of biphenyl heptanoids.

\subsubsection{Aryl-Aryl Bond Formation via Photochemical Cyclization}

Whiting and Wood employed photo-induced radical cyclization for the synthesis of myricanol $[27,151]$. The irradiation of the bromide $\mathbf{7 0}$ in EtOH in the presence of $\mathrm{NaOH}$ with a 252 nm wavelength light for 30 min afforded 72 in $10 \%$, which could be readily converted to myricanol. However, an additional heptanoid 73, expected to be formed via C,O-coupling in 71, was not observed. The starting compound $\mathbf{7 0}$ were prepared from $\mathbf{5 2}$ by the method described for 53a [152].

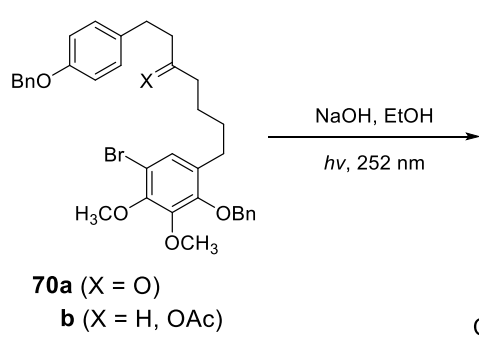

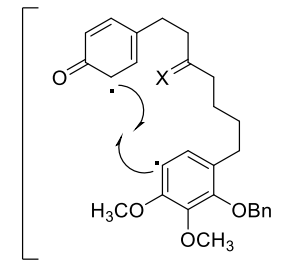

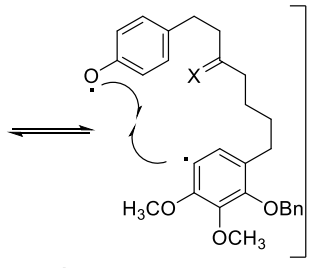

71

C-C coupling

C-O coupling
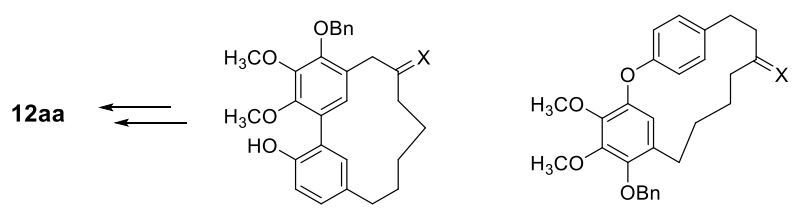

72a $(10 \%)$

b $(10 \%)$

73 (not formed) 


\subsection{Total Synthesis of Cyclic Diphenyl Ether Heptanoids}

The formation of diphenyl ether has long been challenging especially in the chemistry of polypeptide macrocycles and, therefore, led to the development of a couple of practical methods, such as oxidative coupling of phenols [159], the $S_{N} A r$ reaction [160], the Ullmann reaction [161,162], and others $[163,164]$.

\subsubsection{Formation of Macrocycles via Oxidative Coupling}

The first attempt for the synthesis of diphenyl ether heptanoid comes from the biomimetic oxidative coupling of diarylheptanoid $\mathbf{7 6} \mathbf{b}$ with $\mathrm{Tl}\left(\mathrm{OCOCF}_{3}\right)_{3}$ to yield a trace amount of the corresponding diarylheptanoid 77 [27]. Reactions with $\mathrm{K}_{3} \mathrm{Fe}(\mathrm{CN})_{6}, \mathrm{Ag}_{2} \mathrm{O}, \mathrm{MnO}_{2}$ and $\mathrm{VOCl}_{3}$ produced only tars and thus no further applications were reported. Under these conditions, only $\mathrm{C}, \mathrm{O}$-coupling was proceeded to lead diphenyl ether heptanoid 77 unlike the result previously described above for 72a. The prerequisite 76a was prepared via Grignard reaction of 4-(2-benzyloxy-3,4-dimethoxyphenyl)butyl bromide (51) with 3-(4-benzyloxyphenyl)propanal (74), followed by hydrogenolysis.

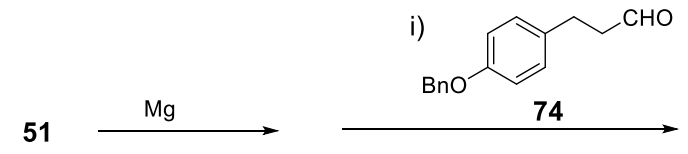

ii) $\mathrm{NH}_{4} \mathrm{Cl}$

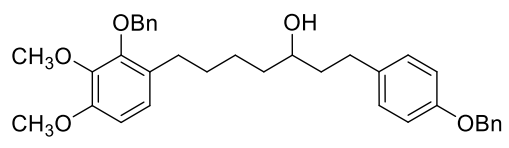

75

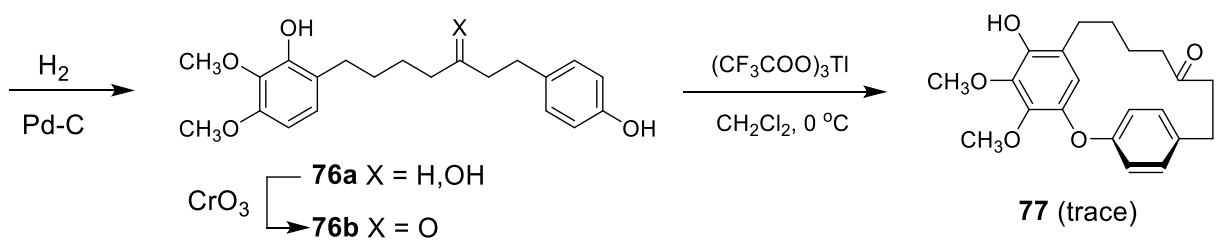

Recently, Salih and Beaudry examined various oxidative cyclization conditions for acerogenin $\mathrm{G}$ (81) [165] and identified the classical phenoxy radical-forming agent $\mathrm{PbO}_{2}$ in $\mathrm{HOAc}$ [166] as the oxidant of choice. Oxidative coupling of $\mathbf{8 1}$ with $\mathrm{PbO}_{2}$ in $\mathrm{HOAc}$, thus, resulted in cyclization with concomitant oxidative hydroxylation of the diphenyl ether and with esterification of a resident phenol, leading to acetyl pterocarine (82a) and its regioisomer (82b) in a ratio of approximately 3:1. Interestingly, the reaction was completely chemoselective and this no oxidative $\mathrm{C}-\mathrm{C}$ coupling product (i.e., acerogenin E) was observed. Although the yield was not somewhat low, one promising result is that $40 \%$ of the starting acerogenin $G(\mathbf{8 1})$ was recoverable. The subsequent hydrolysis of 70a afforded pterocarine (26b). The Horner-Wadsworth-Emmons reaction of phosphonate 78 with aldehyde $\mathbf{7 9}$ gave diene $\mathbf{8 0}$ in $88 \%$ yield, which was then subjected to catalytic hydrogenation to lead to starting acerogenin $\mathrm{G}(\mathbf{8 1})$ in $96 \%$ yield.

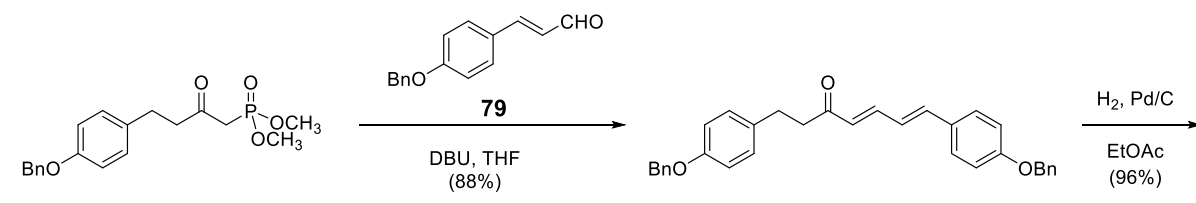

78<smiles>O=C(CCCCc1ccc(O)cc1)CCc1ccc(O)cc1</smiles>

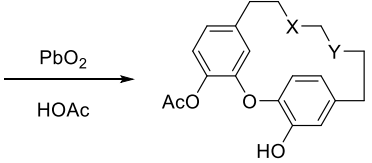

82a $\left(X=\mathrm{CO}, \mathrm{Y}=\mathrm{CH}_{2}\right)(20 \%)$ b $\left(X=\mathrm{CH}_{2}, \mathrm{Y}=\mathrm{CO}\right)(7 \%)$
80

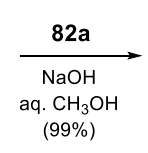

(99\%)

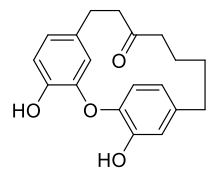

$(+/-)$-pterocarine (26b) 


\subsubsection{Formation of Macrocycles via $S_{N}$ Ar Reaction}

The first successful synthesis of acerogenin C (25b) included an intramolecular nucleophilic aromatic substitution $\left(\mathrm{S}_{\mathrm{N}} \mathrm{Ar}\right)$ macrocyclization of 88a to yield 89 [167]. In order to undertake such an $S_{N} A r$ reaction efficiently, one or more strong electron withdrawing groups, such as a nitro group, are required at the proper positions [168]. The removal of the nitro group after the cyclization requires a couple of additional steps: the reduction of nitro to amine, followed by Doyle's one-step deamination [169] to provide O-methylacerogenin C (90a). $\mathrm{AlCl}_{3}$ catalyzed demethylation of 90a afforded acerogenin $C(\mathbf{2 5 b})$. The reaction of acerogenin $C(\mathbf{2 5 b})$ with 2,3,4,6-tetrabenzoylglucopyranosyl bromide in the presence of $(n-\mathrm{Bu})_{4} \mathrm{NBr}$ provided glycoside 91 with $93 \%$ yield, of which subsequent saponification resulted in aceroside IV (25a) in $95 \%$ yield. The starting linear heptanoid 88a was prepared by employing methyl acetoacetate ester synthesis. Dicarbanion generated from methyl acetoacetate (83) by LDA (2.1 equiv) was alkylated with 84 to give 85, which was then subjected to 2nd alkylation with 86 in presence of $\mathrm{NaH}$ (1 equiv.) to provide $\mathbf{8 7}$. Deprotection of the isopropyl group of $\mathbf{8 7}$, followed by decarboxylation under acidic conditions, resulted in a linear diarylheptanoid 88.

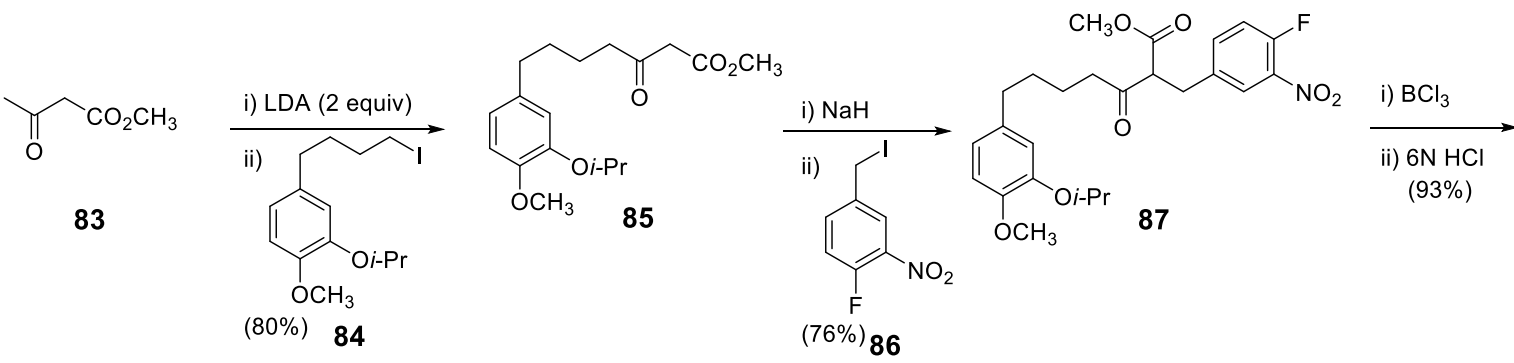<smiles>COc1cc(CCC(=O)CCCCc2ccc(OC)c([N+](=O)[O-])c2)ccc1F</smiles>

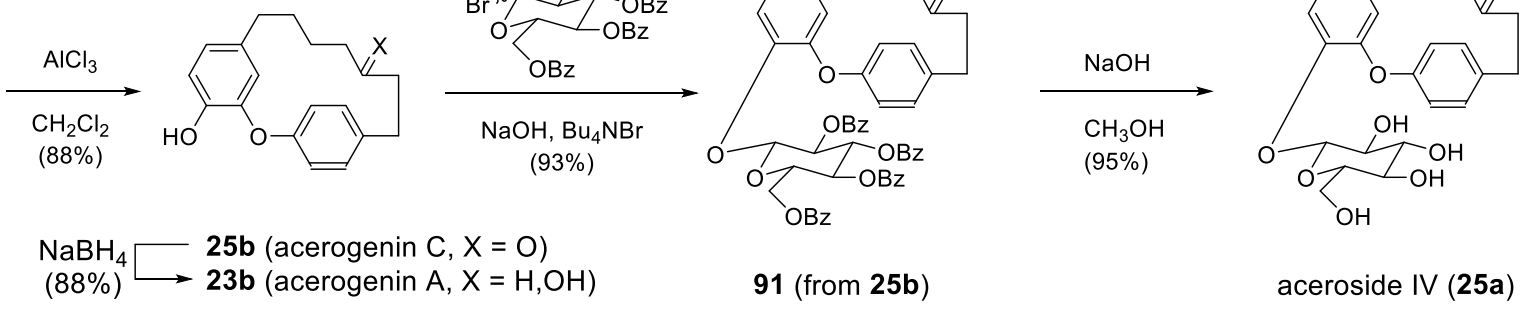

Such a strategy was later employed for the synthesis of acerogenins B and L by using $\mathbf{7 1 b}$ as a starting material [170], which was similarly prepared from 92.<smiles>CC#CCC#CCC(=O)CCc1ccc(OC)c(O)c1</smiles>

92<smiles>COc1ccc(CCC(=O)CCCCc2ccc(F)c([N+](=O)[O-])c2)cc1O</smiles><smiles>C1#CCC#C1</smiles>

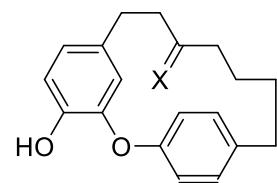

$88 b$ 


\subsubsection{Formation of Macrocycles via Intramolecular Ullmann Ether Synthesis}

Keserü et al. has reported a synthesis of acerogenin A and C via an intramolecular Ullmann ether synthesis [171]. The linear diarylheptanoid 95 was heated at $130{ }^{\circ} \mathrm{C}$ in the presence of $\mathrm{CuBr}-\mathrm{Me}_{2} \mathrm{~S}$ and $t$-BuOK to afford the corresponding $O$-methylacerogenin $C(90 a)$ in $16 \%$ yield, which was then converted to acerogenin $C(\mathbf{2 5 b})$ via demethylation by pyridinium chloride. The subsequent reduction of $\mathbf{2 5} \mathbf{b}$ by $\mathrm{NaBH}_{4}$ led to acerogenin $\mathrm{A}(\mathbf{2 3 b})$. The prerequisite starting compound 95 was prepared by Wittig reaction of 93 with 4-iodobenzaldehyde and the subsequent catalytic hydrogenation of the double bond in 94 .

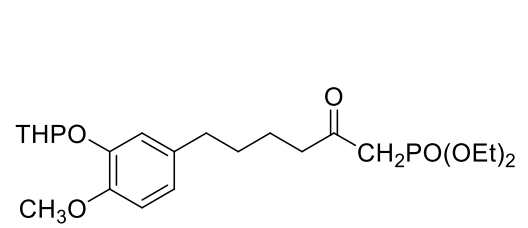

93

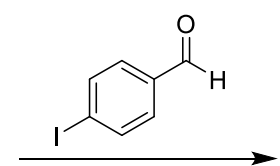<smiles>COc1ccc(CCCCC(=O)/C=C/c2ccc(I)cc2)cc1O</smiles>

94

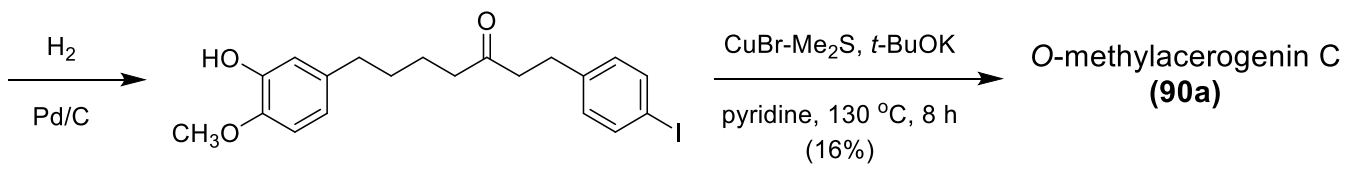

95

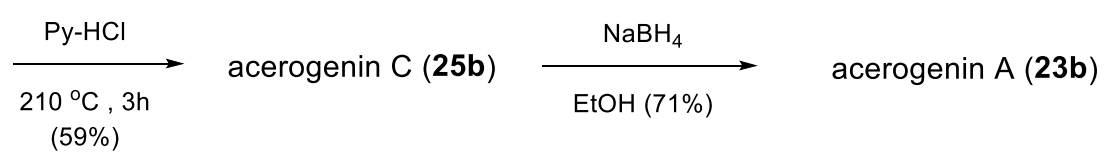

Jahng and his coworkers [172] employed the same methodology for the synthesis of a series of acerogenins; the linear diarylheptanoids were prepared by employing a series of cross aldol condensations. Retrosynthetic analysis of the methods shown in Scheme 2 revealed that such a method holds the advantage that two types of acerogenins such as acerogenin $\mathrm{C}$ and $\mathrm{L}$, can be prepared by the same reaction sequence via starting compounds of 4-halo-3-hydroxybenzaldehydes (96 series) and 3-halo-4-hydroxybenzaldehydes (97 series), respectively.

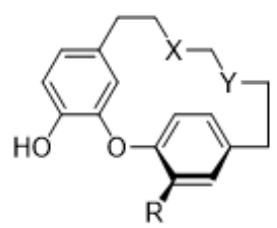

25b $\mathrm{X}=\mathrm{CH}_{2}, \mathrm{Y}=\mathrm{C}=\mathrm{O}, \mathrm{R}=\mathrm{H}$ (acerogenin $\mathrm{C}$ )

26a $X=\mathrm{C}=\mathrm{O}, \mathrm{Y}=\mathrm{CH}_{2}, \mathrm{R}=\mathrm{H}$ (acerogenin $\mathrm{L}$ )

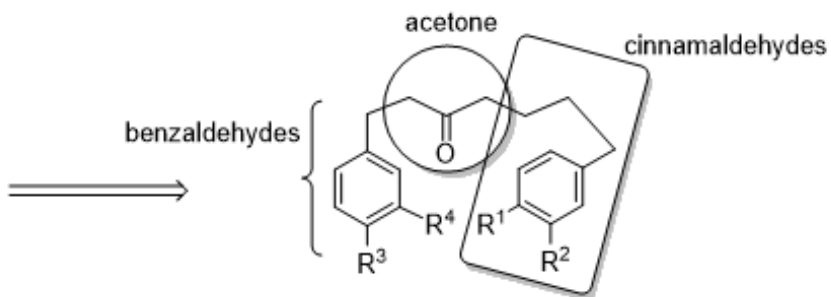

$96\left(R^{2}=\right.$ Halogens and $\left.R^{3}=O H\right)$

$97\left(R^{4}=\right.$ Halogens and $\left.R^{1}=O H\right)$

Scheme 2. Retrosynthetic analysis for the synthesis of acerogenins.

The Claisen-Schmidt condensation of 98a with 4-benzyloxybenzaldehyde gave diarylheptenoid 99a, which was then subjected to catalytic hydrogenation to yield 96a. In the classical Ullmann reaction condition, $\mathrm{CuO}-\mathrm{K}_{2} \mathrm{CO}_{3}$ in pyridine was applied to diarylheptanoid $96 \mathrm{a}$ to yield $\mathrm{O}$-methylacerogenin $\mathrm{C}$ (90a), from which the cleavage of methyl by treating $\mathrm{AlCl}_{3}$ afforded acerogenin $\mathrm{C}(\mathbf{2 5 b})$. It is noteworthy that the application of $50 \mathrm{psi} \mathrm{H}_{2}$ to $99 \mathrm{~b}$ in the presence of $10 \% \mathrm{Pd} / \mathrm{C}$ resulted in the reduction of a double bond, the hydrogenolysis of benzyl ether as well as the $\mathrm{Ar}-\mathrm{Br}$ bond. However catalytic 
hydrogenation at $1 \mathrm{~atm} \mathrm{H}_{2}$ for $10 \mathrm{~h}$ led to the reduction of a double bond and the hydrogenolysis of benzyl ether without cleavage of the $\mathrm{Ar}-\mathrm{Br}$ bond. The subsequent intramolecular Ullmann reaction cyclization reaction followed by demethylation afforded acerogenin L (26a).<smiles>COc1ccc(CCCCC(C)=O)cc1Br</smiles>

98a

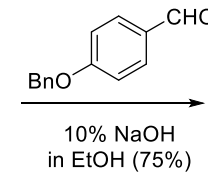<smiles>COc1ccc(CCCCC(=O)/C=C/c2ccc(OCc3ccccc3)cc2)cc1Br</smiles>

$99 a$<smiles>COc1ccc(CCCCC(=O)CCc2ccc(O)cc2)cc1Br</smiles>
$\underset{\text { pyridine }(76 \%)}{\stackrel{\mathrm{CuO}, \mathrm{K}_{2} \mathrm{CO}_{3}}{\longrightarrow}}$

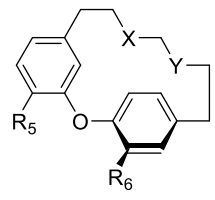

90a $\left(X=\mathrm{CH}_{2}, Y=\mathrm{C}=\mathrm{O}, \mathrm{R}^{5}=\mathrm{OCH}_{3}, \mathrm{R}^{6}=\mathrm{H}\right)$ from 96a

b $\left(X=\mathrm{C}=\mathrm{O}, \mathrm{Y}=\mathrm{CH}_{2}, \mathrm{R}^{5}=\mathrm{H}, \mathrm{R}^{6}=\mathrm{OCH}_{3}\right)$ from $97 \mathbf{a}$ $\uparrow \mathrm{CuO}, \mathrm{K}_{2} \mathrm{CO}_{3}(49 \%)$<smiles>COc1ccc(CCC(=O)CCCCc2ccc(O)cc2)cc1Br</smiles><smiles>CC(=O)CCCCc1ccc(OCc2ccccc2)cc1</smiles>

$98 b$

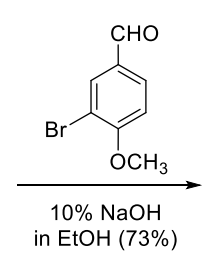

$(73 \%)$

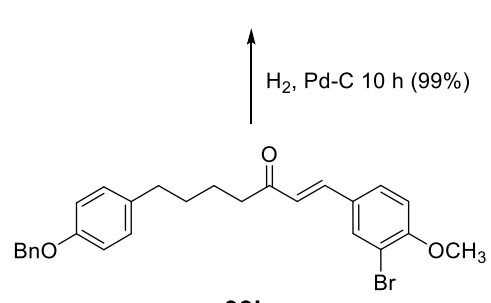

99b

The same reaction sequence was applied to the synthesis of galeon and pterocarine [173]. The catalytic reduction of the double bond of $99 \mathbf{c}$ afforded $\mathbf{9 7 b}$. The hydrogenation reaction time is critical for keeping the benzyl group: it can be minimized by stopping the reaction in $2.5 \mathrm{~h}$ at room temperature. The intramolecular Ullmann reaction of $97 \mathrm{~b}$ with $\mathrm{CuO} / \mathrm{K}_{2} \mathrm{CO}_{3}$ in pyridine afforded 90c, from which hydrogenolysis gave galeon (26c) and through subsequent demethylation of 26c afforded pterocarine (26d). The starting 99c was prepared by the same synthetic sequence employed above for 99a,b by Claisen-Schmidt condensation of 6-(4-hydroxy-3-methoxyphenyl)hexan-2-one with 4-benzyloxy-3-bromobenzaldehyde.<smiles>COc1cc(CCCCC(=O)/C=C/c2ccc(OC(Br)(Br)c3ccccc3)c(Br)c2)ccc1O</smiles>

99c

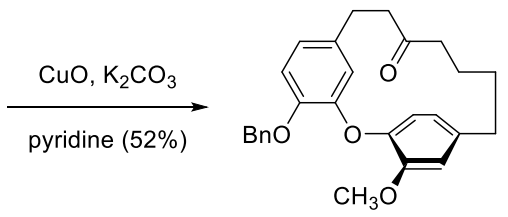

$90 \mathrm{c}$

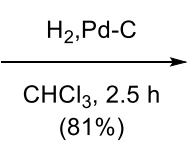

$(81 \%)$<smiles>COc1cc(CCCCC(=O)CCc2ccc(OCc3ccccc3)c(Br)c2)ccc1O</smiles>

97b

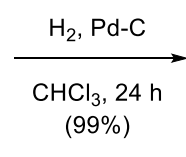

$(99 \%)$

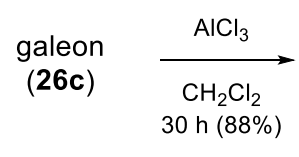

pterocarine

(26d) 
The same methodology was applied to 100a to afford 101, which gave the corresponding didemethylated product (28) in $42 \%$ yield by $\mathrm{AlCl}_{3}$-mediated cleavage of methyl ether moiety [174]. The ${ }^{1} \mathrm{H}$ NMR spectrum of the product is not identical to that of engelhardione reported previously [120] and but did match that of pterocarine (vide ante). Based on these data, Shen and Sun revised the structure of engelhardione to be pterocarine [174] (vide ante). The starting 100a was prepared from 6-(3-hydroxy-4-methoxyphenyl)hexan-2-one and 3-bromo-4-methoxybernzaldehyde via Claisen-Schmidt condensation followed by catalytic hydrogenation.<smiles>COc1ccc(CCC(=O)CCCCc2ccc(OC)c(Br)c2)cc1O</smiles>

$100 a$

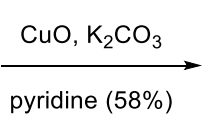

pyridine $(58 \%)$

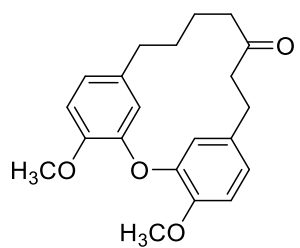

101

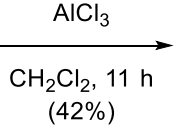

(28, proposed structure)

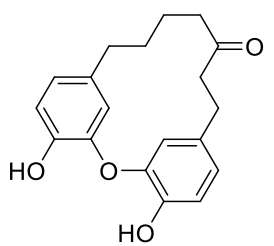

engelhardione

Recently, Salih and Beaudry reported the synthesis of a series of diphenyl ether-type diarylheptanoids such as myricatomentogenin (26e) and jugcathanin (juglanin A, 26f) [175]. The Horner-Wadsworth-Emmons reaction of 102 with phosphonate 103 yielded hept-3,5-dien-2-ones 104. The catalytic hydrogenation of $104 a$ and $104 b$, followed by cyclization under Ullmann reaction conditions provided cyclophanes $90 \mathrm{~d}$ and $90 \mathrm{e}$, respectively. The selective cleavage of the isopropyl ether using $\mathrm{BCl}_{3}$ completed the synthesis of myricatomentogenin (26e) and jugcathanin (26f).

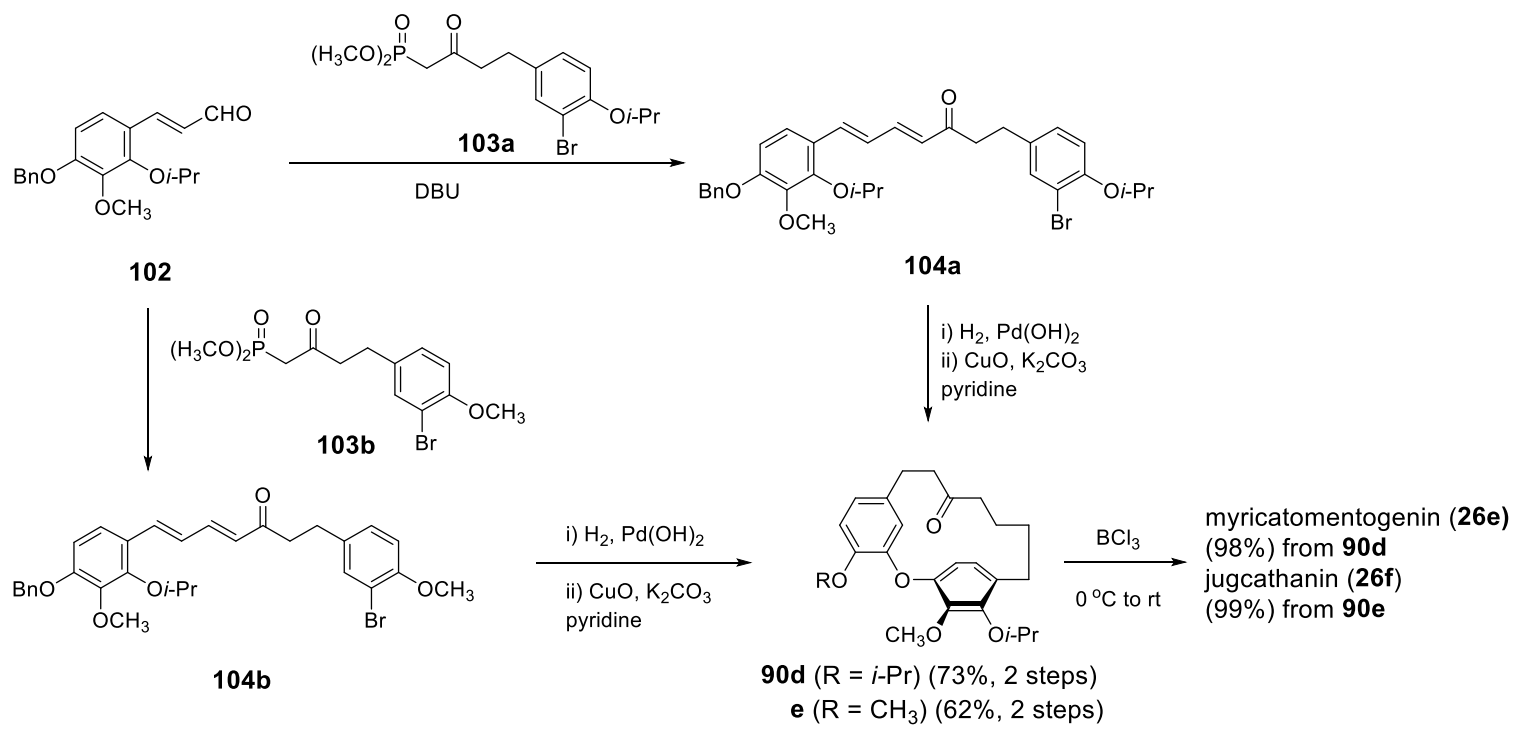

The starting cinnamaldehyde $\mathbf{1 0 2}$ was prepared in 4 steps from the corresponding coumarins via methanolysis, isopropyl ether formation, diisobutylaluminum hydride reduction and Dess-Martin oxidation.

The last part of diphenyl ether-type heptanoids describes the synthesis of garuganins and garugamblins. Beaudry and his coworkers reported an elegant synthesis and conformational dynamics of these series, in which they employed 1,7-diarylhept-3,5-diones as key intermediates [128,129]. To the lithium enolate of 105 was added an aldehyde 106 to produce an aldol product 107 . The oxidation of $\beta$-hydroxy ketone using IBX gave 1,3-diketone 108, which was subjected to selective debenzylation by $\mathrm{BCl}_{3}$ at $-78{ }^{\circ} \mathrm{C}$ to give 109. An intramolecular Ullmann reaction using stoichiometric $\mathrm{CuO}$ in pyridine at the elevated temperature gave the cyclized product $\mathbf{1 1 0}$ in $38 \%$ yield. Methyl ether was achieved by treating $\mathrm{CH}_{3} \mathrm{OH}$ in presence of $p-\mathrm{TsOH}$. In addition, the treatment of $\mathbf{2 8 b}$ with 
$\mathrm{CH}_{3} \mathrm{I}$ afforded garuganin VI (31). Most of garuganins and garugamblins were prepared by using dihydrocinnamaldehyde derivatives 106 with appropriate substituents.<smiles>CC(=O)CCc1ccc(Br)cc1</smiles>

105

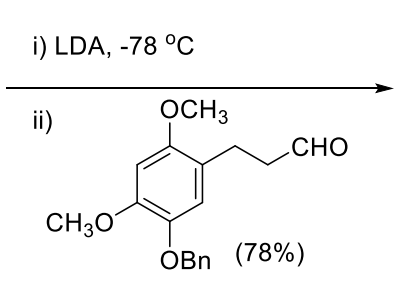

106<smiles>COc1cc(OC)c(OCC(=O)CC(O)CCc2ccc(Br)cc2)cc1CCC(=O)CC(C)(Br)Br</smiles>

107<smiles>COc1cc(OC)c(OCc2ccccc2)cc1CCC(=O)CC(=O)CCc1ccc(Br)cc1</smiles><smiles>COc1cc(OC)c(Oc2ccc(CCC(O)=CC(=O)CCc3ccccc3)cc2OC)cc1OC</smiles>

110

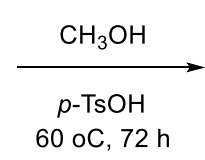

$(17 \%)$

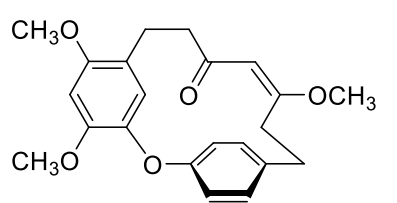

garuganin I (28b)

Finally tedarene A (41a) was prepared by the dehydration of 116, which was prepared 4 steps from 3-(4-methoxyphenyl)propanal (111) and (4-(but-3-yn-1-yl)phenoxy)(t-butyl)dimethylsilane (112) [6] via Ullmann cyclization as a key reaction. An acetylide generated from 112 was reacted with 113 to afford the expected propargyl alcohol 113. The optimized, controlled (6 atm of $\mathrm{H}_{2}$ for $18 \mathrm{~h}$ ), and stereospecific hydrogenation of alkyne in $\mathbf{1 1 3}$ produced a Z-alkene (114a), which was then subjected to deprotection of the $t$-butyldimethylsilyl (TBS) group by tetra- $n$-butylammonium fluoroborate (TBAF) to give 114b. The intramolecular Ullmann reaction of $\mathbf{1 1 4 b}$, followed by demethylation with NaSEt in refluxing DMF, afforded 115. Kozikowski's dehydration method using methanesulfonyl chloride in the presence of $\mathrm{NEt}_{3}$ in $\mathrm{CH}_{2} \mathrm{Cl}_{2}$ from $0{ }^{\circ} \mathrm{C}$ to room temperature [176] was applied to 115 to allow the elimination reaction to proceed, along with the unavoidable mesylation of the phenol leading to a mixture of diene $E, Z$ - and $E, E$-isomers $116 \mathbf{a}$ and $\mathbf{1 1 6 b}$ in a 6:4 ratio (as determined by ${ }^{1} \mathrm{H}$ NMR) in $80 \%$ yield. Hydrolysis of the mesylates was finally accomplished by aq. $\mathrm{NaOH}$ in an optimized 1:1 $\mathrm{CH}_{3} \mathrm{OH}$ /dioxane mixture at $60{ }^{\circ} \mathrm{C}$, in $67 \%$ yield. Preparative HPLC chromatography using a chiral-phase column in direct phase allowed the separation of the two macrocycles, tedarene A (41a) and its isomer $\mathbf{4 1 b}$ in 38.5\% and 23\% isolated yields, respectively, of which the structures were confirmed by X-ray structure analysis. The starting compound $\mathbf{1 1 1}$ was prepared in 2 steps from 3-(4-benzyloxyphenyl)propanal by regioselective bromination on the aromatic ring by $\mathrm{Br}_{2}$ in presence of $\mathrm{AlCl}_{3}$, and the subsequent IBX oxidation of the resulting alcohol led to the aldehyde. 
<smiles>COc1ccc(CCC=O)cc1Br</smiles>

111

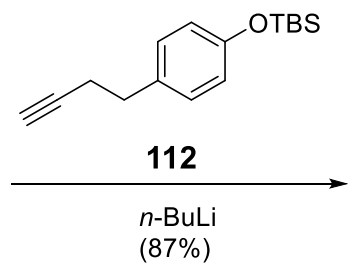
113
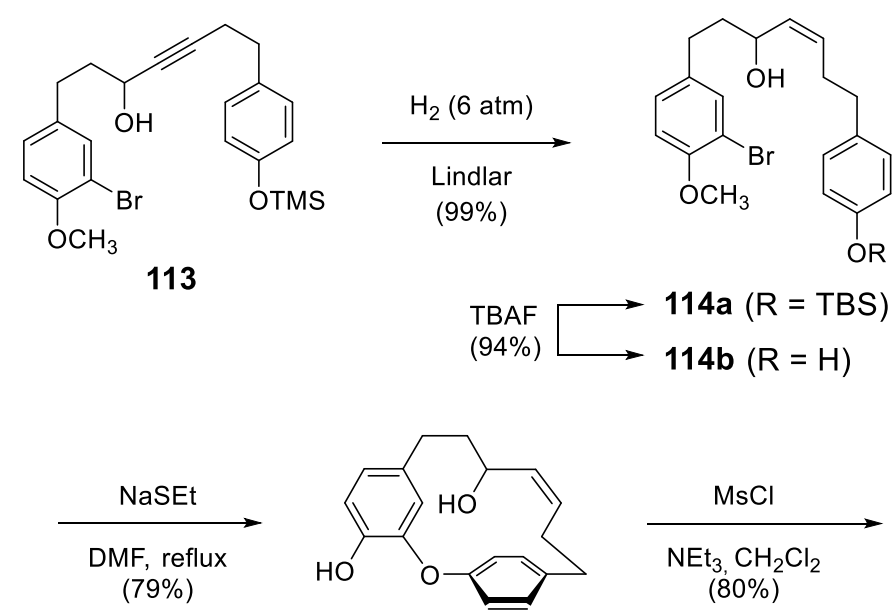

116

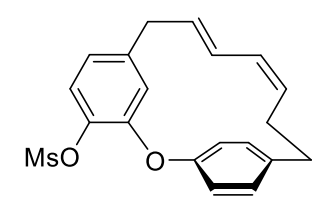

$117 a$

115

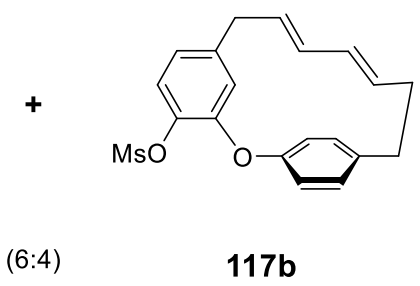

aq. $\mathrm{NaOH}, \mathrm{CH}_{3} \mathrm{OH} /$ dioxane $(67 \%)$

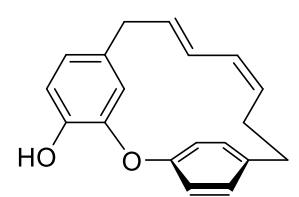

$41 \mathrm{a}$

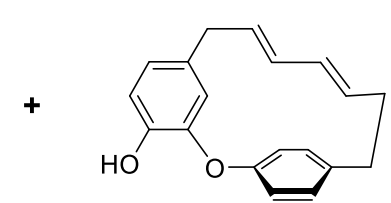

41b

$(6: 4)$

\subsubsection{Formation of Macrocycle via Formation of Heptane Skeleton}

Via Wurtz and/or Wittig Reactions

The Wurtz and Wittig reactions were employed for the synthesis of garugamblin I, II, and III. Vermes et al. reported a synthesis of garugamblin I [177]: The Wittig reaction of the biaryl aldehyde 118 with 119 in the presence of $t$-BuOK afforded the alkene compound 120, which was then converted to the dibromide compound $\mathbf{1 2 1}$ by a three-step reaction sequence. The radical anion induced by intramolecular Wurtz reaction with synchronistical cleavage of isoxazole provided $\beta$-enaminoketone 122 in 16\% yield, where the intramolecular hydrogen bonding may force to adapt (Z)-alkene. The subsequent hydrolysis followed by methylation of enol with diazomethane led to two regioisomeric ( $Z$ )-enol ethers 123a and 123b Compound 123a slowly isomerized to natural garugamblin I (28a) by simply standing in a chloroform solution for 2 weeks. The same isomerization has also been observed for 123b to 124. These observation may imply that (Z)-isomers [(E)-heptenes, 28a and 124] are somewhat more stable than the corresponding $(E)$-isomers [ $Z$ )-heptenes, 123a and 123b, respectively. 
<smiles>COc1ccc(C=O)cc1Oc1ccc(C(C)=O)cc1</smiles>

118

$$
+\mathrm{CO}_{2} \mathrm{CH}_{3} \stackrel{\substack{\mathrm{DMSO} \\(80 \%)}}{\stackrel{\mathrm{KO}-\mathrm{Bu}}{\longrightarrow}}
$$

119<smiles>COc1ccc(CCc2cc(CBr)no2)cc1Oc1ccc(CBr)cc1</smiles>

121<smiles>COC(=O)c1ccc(Oc2cc(/C=C/c3cc(C(C)=O)no3)ccc2OC)cc1</smiles>

120

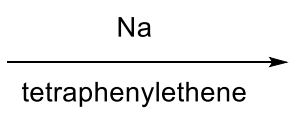<smiles>COc1ccc(CCC(=O)/C=C(\N)CCc2ccc(OC)cc2)cc1</smiles>

122

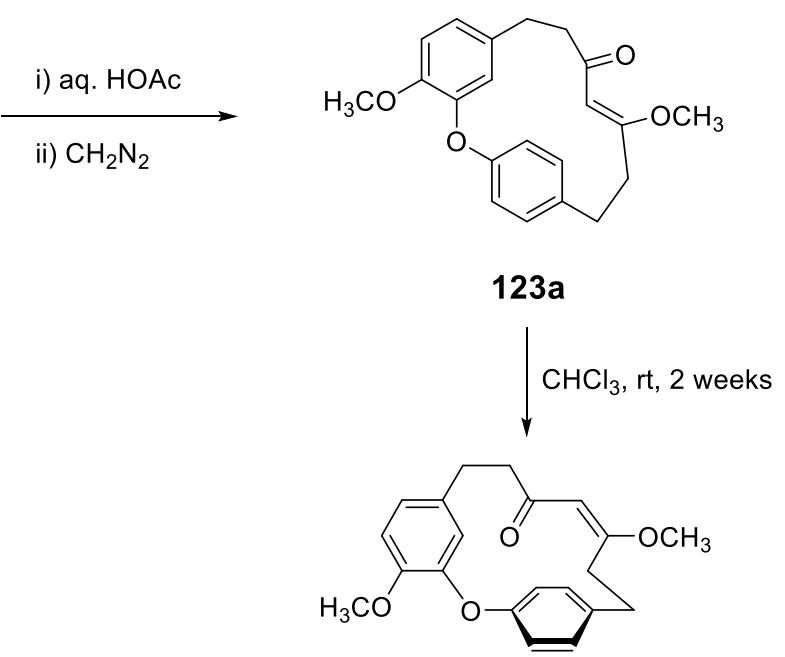

garugamblin 1 (28a)<smiles>COC(=O)CCc1ccc(OC)c(Oc2ccc(CCC(=O)C=C(CO)OC)cc2)c1</smiles>

123b

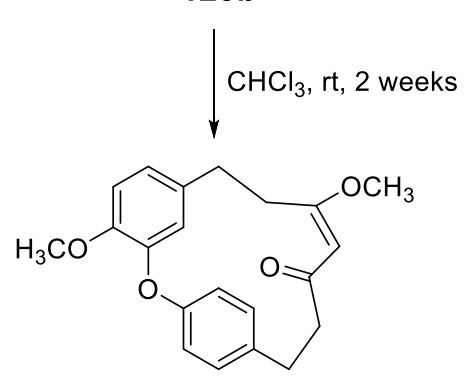

124

On the other hand, the same group employed a Wittig reaction for the synthesis of garuganin III (32) [128]. The intramolecular Wittig reaction was pursued by the addition of $t$-BuOK to a dilute solution of $\mathbf{1 2 5}$ in DMF to produce macrocycle 126. The catalytic hydrogenation over $\mathrm{PtO}_{2}$ doped with Raney nickel saturated the double bond and cleaved isoxazole ring to give the enaminoketone 127. The hydrolysis of $\mathbf{1 2 7}$ quantitatively resulted in the corresponding ketoenol, which was then methylated with diazomethane to yield garuganin III (32) with a mixture of its region- and stereoisomers. Based on the synthesis, the originally proposed structure was revised as shown. The same group employed identical synthetic methodology for the synthesis of garugamblin-2 (31) [178]. 
<smiles>COc1cc(CCc2cc(Cc3ccccc3)no2)cc(Oc2ccc(C=O)cc2)c1OC</smiles>

125<smiles>COc1cc(CCC(=O)/C=C(\N)CCc2ccc(Oc3ccccc3)cc2)cc(OC)c1O</smiles>

127<smiles>COc1cccc(CCc2cc(/C=C\c3ccc(Oc4cc(O)c(OC)c(OC)c4)cc3)no2)c1</smiles>

126<smiles></smiles>

garuganin III (32)

\section{Via Ring-Closing Metathesis}

Ring-closing metathesis has been used as a powerful tool for macrocyclization [147]. The intermolecular Ullmann reaction of 128 and 129 afforded the prerequisite precursor 130, of which the ring closure metathesis with a variety of Grubbs' catalysts for the synthesis of ovalifoliolatin B (35b) [179] did not proceed at all. On the other hand, it is worth noting that an intermolecular ring closure metathesis of $\mathbf{1 2 8}$ and $\mathbf{1 2 9}$ led to an inseparable mixture of stereoisomers of the precursor 131, which was then $\mathrm{CuO}$ mediated Ullmann cyclization to provide ovalifoliolatin B (35b) and its cis-isomer 132 in a ratio of 13:1. The catalytic hydrogenation of these two isomers, followed by demethylation by $\mathrm{AlCl}_{3}$ afforded acerogenin $\mathrm{C}(\mathbf{2 5 b})$. All attempts for the synthesis of cyclic diarylheptanoids employing ring closing metathesis have, thus far, failed implying the scope of such a reaction for the application towards the synthesis of cyclic diaryl ether heptanoids.<smiles>C=CCCC(=O)CCc1ccc(Br)cc1</smiles>

128<smiles>C1CCCC1</smiles><smiles>COc1ccc(/C=C/CCC(=O)CCc2ccc(Br)cc2)cc1O</smiles>

131
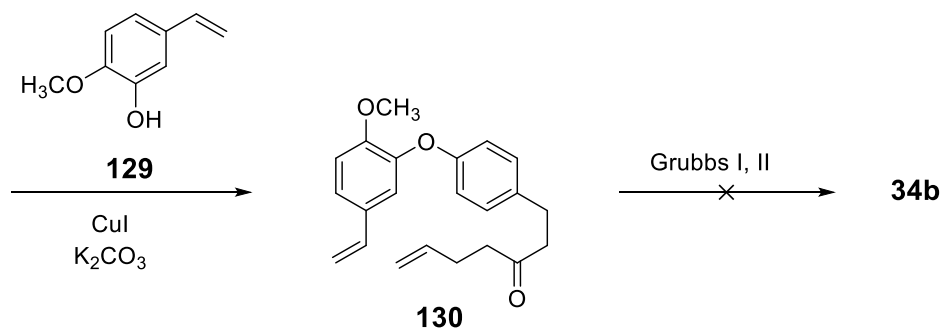

ovalifoliolatin B

(35b)

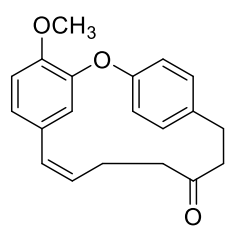

132

\subsubsection{Enantioselective Synthesis of Diaryl Ether Heptanoids}

Two types of enantioselective reactions, such as enantioselective Ullmann ether coupling and chiral phase-transfer-catalyzed atropselective diaryl ether formation via $\mathrm{S}_{\mathrm{N}}$ Ar reaction, have been reported. 
Salih and Beaudry reported the first asymmetric syntheses of (-)-myricatomentogenin, $(-)$-jugcathanin, (+)-galeon, and (+)-pterocarine by enantioselective Ullmann cross-coupling reaction [180]. The intramolecular coupling reaction was evaluated using enantiopure ligands known to accelerate the Ullmann reaction and some other privileged ligand structures. Cu-catalyzed cross coupling of $97 \mathrm{c}$ in the presence of BINOL-type ligands would lead enantioselectivity with low chemical yields. Among the 21 tested ligands, $N$-methyl-L-proline did lead to not only increased chemical yield up to $41 \%$ but also enantioselectivity up to $68: 32$ in the presence of $20 \mathrm{~mol} \%$ CuI. Variations in the $\mathrm{N}$-alkyl group, the ring size, and the carboxylic acid functionality did not significantly improve the yield or enantioselectivity of the reaction. The survey of a variety of inorganic and organic bases in the Ullmann coupling indicated that use of $\mathrm{K}_{3} \mathrm{PO}_{4}$ instead of $\mathrm{Cs}_{2} \mathrm{CO}_{3}$ could be the conditions of choice with a higher enantiomeric ration without a significant loss of chemical yield (see Table 7). Thus, enantioselective cross Ullmann coupling of $97 \mathrm{c}$ was pursued under the optimized reaction conditions [CuI (20 mol \%), $N$-methyl-L-proline ( $40 \mathrm{~mol} \%), \mathrm{K}_{3} \mathrm{PO}_{4}$ (2 equiv.) in dioxane] afforded (pR)-90f with 72:28 er. Although they did not find any better system, such enantiomeric ratio can be improved to 92:8 er by recrystallization. The partial and complete demethylation of $(\mathrm{p} R)-90 \mathrm{f}$ afforded (+)-galeon $(43 \%,(92: 8 \mathrm{er})$ and $(+)$-pterocarine $(45 \%, 92: 8)$, respectively.<smiles>COc1ccc(CCCCC(=O)CCc2ccc(O)c(Br)c2)cc1</smiles>

$97 c$

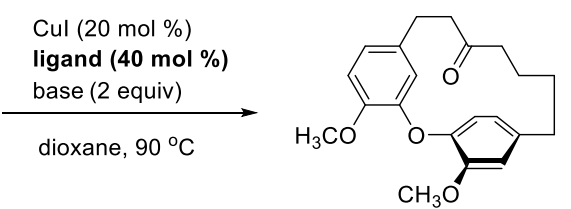

$(p R)-90 f$

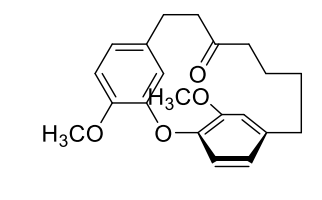

$(\mathrm{p} S)-90 \mathrm{f}$

Table 7. Optimization of reaction conditions.

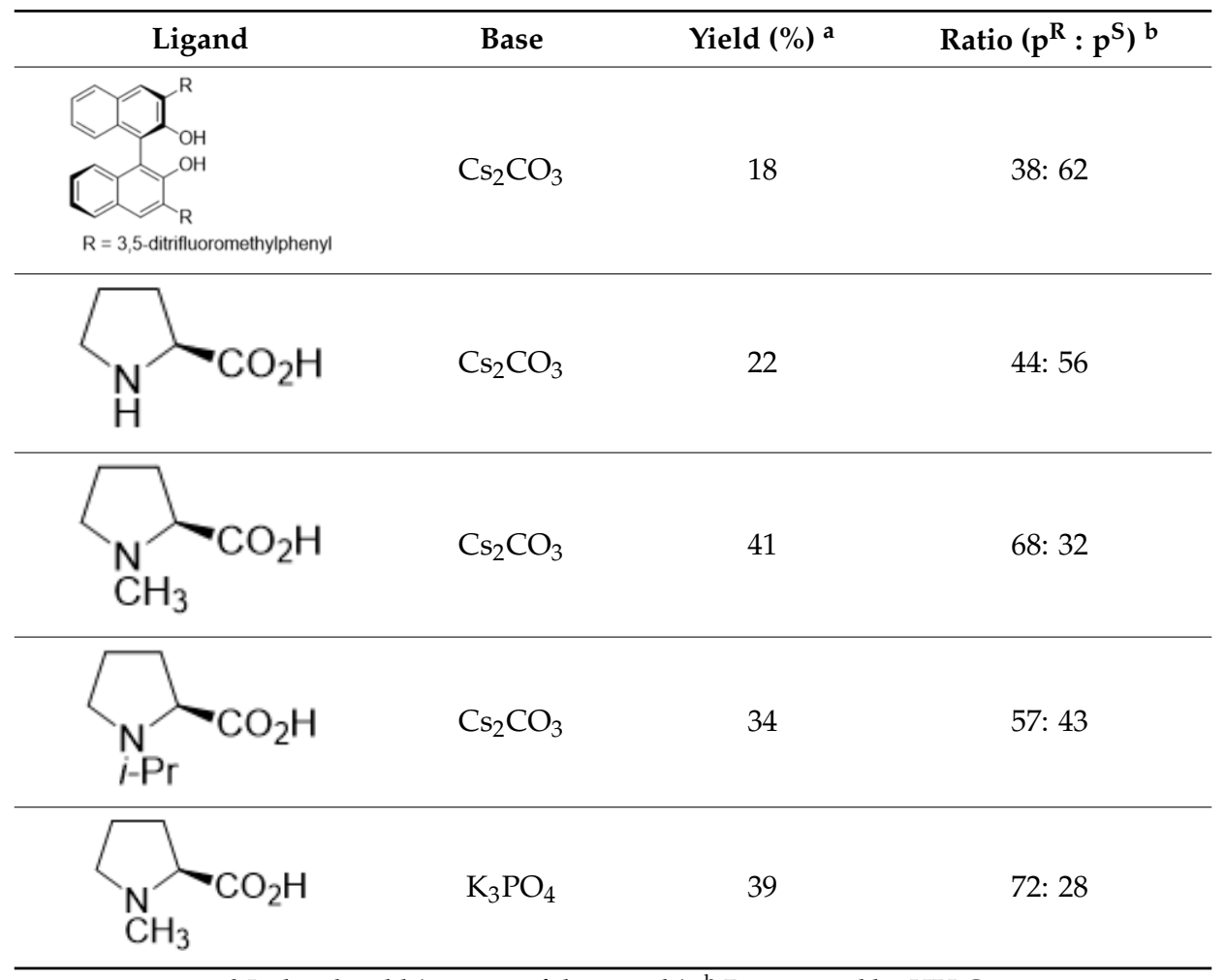

${ }^{a}$ Isolated yield (average of three trials). ${ }^{b}$ Determined by HPLC.

Subsequent Ullmann cross-coupling of $97 \mathrm{~d}$ under optimized conditions [CuI (20\% $\mathrm{mol})$ in the presence of $\mathrm{K}_{3} \mathrm{PO}_{4}$ (2 equiv) and chiral ligand, $N$-methyl-L-proline $\left.(40 \mathrm{~mol} \%)\right]$ afforded $90 \mathrm{~g}$ in moderate yield and with a degree of enantioselectivity (67:33 er). The enantiomeric ratio of cyclophane 
$90 \mathrm{~g}$ was increased up to 82:18 er by additional recrystallization. The treatment of $90 \mathrm{~g}$ with $\mathrm{BCl}_{3}$ gave a 1:1 mixture of the product with one isopropyl group $(\mathbf{9 0 h})$ and (-)-myricatomentogenin (26e) in nearly quantitative yield without any loss in enantioenrichment. Subsequent methylation followed by the removal of isopropyl group in $90 \mathrm{~h}$ provided (-)-jugcathanin (26f) without any loss in enantiomeric ratio.<smiles>COc1ccc(CCC(=O)CCCCc2ccc(O)c(OC)c2O)cc1Br</smiles>

97d

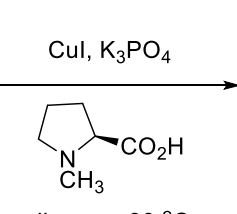

dioxane, $90^{\circ} \mathrm{C}$

$(24 \%)$

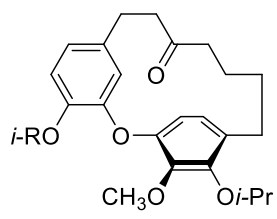

(pR)-90g er 67:33 $\longrightarrow$ recrystallization

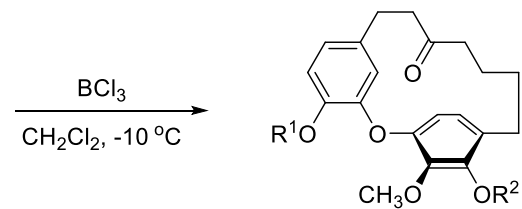

(pR)-90h $\mathrm{R}^{1}=\mathrm{H}, \mathrm{R}^{2}=i-\mathrm{Pr} \longrightarrow$ i) $\mathrm{CH}_{3} \mathrm{I}, \mathrm{K}_{2} \mathrm{CO}_{3}$ (-)-jugcathanin [(-)-26f] $\mathrm{R}^{1}=\mathrm{CH}_{3}, \mathrm{R}^{2}=\mathrm{H} \longleftarrow$ ii) $\begin{array}{r}\mathrm{BCl}_{3}, \mathrm{CH}_{2} \mathrm{Cl}_{2} \\ (84 \%, 2 \text { steps })\end{array}$ er $=82: 18$

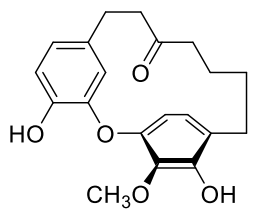

(-)-myricatomentogenin (26e) er $=82: 18$

Alternatively, Ding et al. used the chiral phase transfer-transfer catalyst to induce an asymmetry during intramolecular $\mathrm{S}_{\mathrm{N}} \mathrm{Ar}$ cyclization for the formation of diphenyl ether [181]. They screened the reaction conditions in the presence of various chiral phase-transfer catalysts and found the cinchonine-derived ligand $\mathbf{1 3 3}$ as a phase-transfer catalyst of choice and $20 \% \mathrm{CsOH}$ in toluene as base and solvent (see Table 8) as a best reaction condition.

Table 8. Screening reaction conditions for cyclization of linear diarylheptanoid $\mathbf{8 8 b}$.

\begin{tabular}{|c|c|c|c|}
\hline $88 \mathrm{~b}$ & $\begin{array}{c}\text { reaction conditiona) } \\
\begin{array}{c}\mathrm{rt}, 40 \mathrm{~h} \\
\text { chiral PTC }\end{array}\end{array}$ & $(\mathrm{pS})-89$ & \\
\hline Solvent & Base (aq. Solution) & Yield $(\%)$ b) & $\mathrm{Er}^{\mathrm{c})}$ \\
\hline toluene (5 mL) & $20 \% \mathrm{CsOH}$ & 47 & 87: 13 \\
\hline DMF (5 mL) & $20 \% \mathrm{CsOH}$ & 90 & 50: 50 \\
\hline xylene (5 mL) & $20 \% \mathrm{CsOH}$ & 47 & 82: 18 \\
\hline toluene ( $5 \mathrm{~mL}$ ) & $20 \% \mathrm{CsF}$ & $<10$ & 69: 31 \\
\hline toluene $(5 \mathrm{~mL})$ & $20 \% \mathrm{KOH}$ & 17 & 64: 36 \\
\hline toluene $(5 \mathrm{~mL})$ & $20 \% \mathrm{CsOH}$ & 46 & 89: 11 \\
\hline toluene $(5 \mathrm{~mL}) \mathrm{d})$ & $20 \% \mathrm{CsOH}$ & 80 & 91: 9 \\
\hline toluene $(5 \mathrm{~mL}) \mathrm{d})$ & $20 \% \mathrm{CsOH}$ & 47 & 91.5: 8.5 \\
\hline
\end{tabular}

Therefore, the intramolecular $\mathrm{S}_{\mathrm{N}} \mathrm{Ar}$ reaction of $\mathbf{8 8 \mathbf { b }}$ was explored on a $1 \mathrm{~g}$ scale in the presence of PTC $133(20 \mathrm{~mol} \%), 20 \% \mathrm{CsOH}(\mathrm{aq})$ in toluene at room temperature to afford the desired product (pS)-89 in 81\% yield with an er of 91:9. This er was improved up to 99:1 by recrystallization, with overall 
$62 \%$ yield. In addition, the hydrogenation of $(\mathrm{pS})-89$ provided the corresponding amino compound 134, of which the absolute configuration was determined as $\mathrm{p} S$ by $\mathrm{X}$-ray crystallography. Compound 134 was further converted to a hydroxyl compound $\mathbf{1 3 5}$ without any measurable loss of enantiopurity by using a previously published method [170]. The demethylation of $\mathbf{1 3 5}$ led to pterocarine, which was determined to be (-)-pterocarine by comparison with previously reported data for its enantiomer [25].

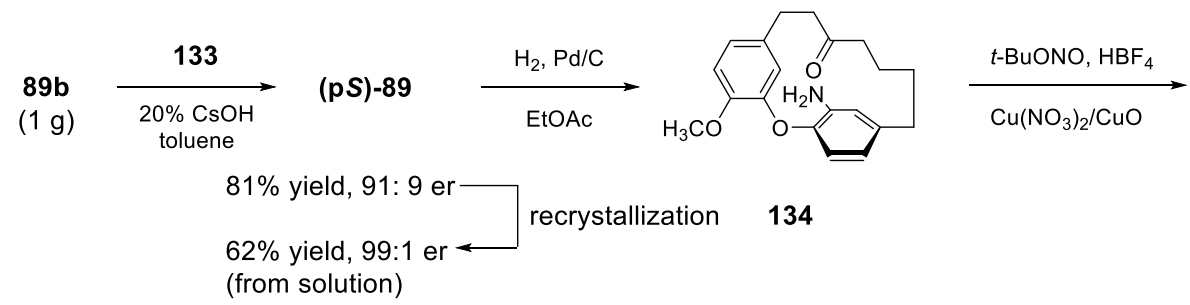

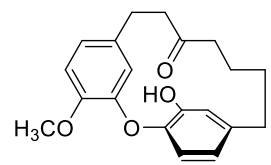

135

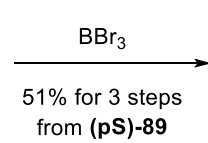

from (pS)-89

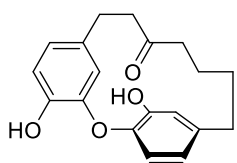

(-)-pterocarine $[(-)-26 b)$

Similarly, the same reaction sequence starting with counter precursor (88c) of $\mathbf{8 8 a}$ afforded the desired compound 136 in 83\% yield, but with only moderate enantioselectivity (69:31 er). However the highly (99:1 er) (-)-enriched 136 could be obtained by simple recrystallization, which then converted to $(-)$-galeon by a simple transformation procedure reported previously.

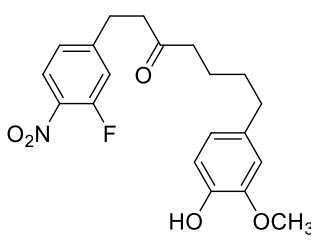

$88 \mathrm{c}$

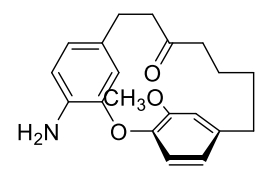

137
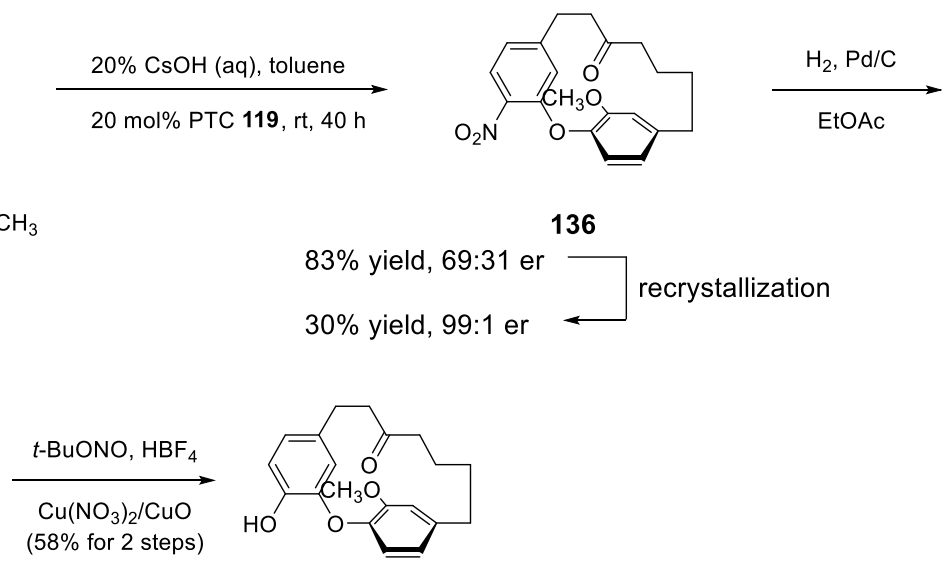

$(-)$-galeon $[(-)-26 c b]$

99:1 er

\section{Conclusion}

Steady progress in the chemistry and biology of cyclic diarylheptanoids has resulted in a diverse range of new structures and new biological activity profiles. Slow but continuous efforts on the synthesis of cyclic diarylheptanoids have led not only to the revision of originally proposed structures, but also to the identification of synthetic methods to establish atropenantiomers. Partial success in the enantioselective synthesis of (-)-myricatomentogenin, (-)-jugcathanin, (+)-galeon, and (+)-pterocarine by Ullmann cross coupling in the presence of chiral ligands and enantioselective synthesis of $(-)$-pterocarine and (-)-galeon by enantioselective $S_{N} A r$ formation in the presence of chiral phase-transfer catalyst may open a door to the development of an enantioselective macrocyclization for diphenyl ether heptanoids. On the other hand, a couple of the enantioselective total syntheses for 
the biaryl macrocycle skeleton via Suzuki-Miyaura cross-coupling and successful enantioselective Suzuki-Miyaura cross-coupling for the synthesis of subclass linear diarylheptanoid, diospongin B [158] may lead to a practical enantioselective synthesis of biphenyl heptanoids.

However, to the best of our knowledge, the structure-activity relationship (SAR) study of cyclic diarylheptanoids is very limited $[80,182,183]$; thus, the identification of good drug candidates has not been fruitful yet. Not only development of a facile enantioselective macrocyclization method but also more intense SAR study have become a prerequisite for realizing such a goal.

Author Contributions: J.G.P. organized the first draft, which was reorganized and refined by Y.J.

Funding: This research was funded by Yeungnam University, grant number [\#215A061026].

Acknowledgments: Financial support from Yeungnam University (2015, \#215A061026) is gratefully appreciated.

Conflicts of Interest: The authors declare no conflict of interest.

\section{References and Note}

1. Although the nomenclature of this system generated from ChemDraw ${ }^{\circledR}$ is 1,2(1,3)-dibenzenacyclononaphane, we used common name system which is more familiar to most of the chemists. Similarly, oxa[7.1]metaparacyclophane was used instead of 2-oxa-1(1,3),3(1,4)-dibenzenacyclodecaphane for type II diaryl ether heptanoids.

2. Keserü, G.M.; Nógrádi, M. The chemistry of natural diarylheptanoids. Stud. Nat. Prod. Chem. 1995, 17, 357-394.

3. Zhu, J.; Islas-Gonzalez, G.; Bois-Choussy, M. Recent progress in isolation, bioactiviy evaluation and total synthesis of diarylheptanoids. Org. Prep. Proced. Int. 2000, 32, 505-546. [CrossRef]

4. Vogel, H.; Pelletier, J. Examen chimique de la racine de curcuma. J. Pharm. 1815, 1, 289-303.

5. Milobedzka, J.; van Kostanecki, S.; Lampe, V. Zur Kenntnis des Curcumins. Ber. Dtsch. Chem. Ges. 1910, 43, 2163-2170. [CrossRef]

6. Maurent, K.; Vanucci-Bacqué, C.; Saffon-Merceron, N.; Baltas, M.; Bedos-Belval, F. Total synthesis of tedarene A. J. Nat. Prod. 2017, 80, 1623-1630. [CrossRef] [PubMed]

7. Bi, W.; Gao, Y.; Shen, J.; He, C.; Liu, H.; Peng, Y.; Zhang, C.; Xiao, P. Traditional uses, phytochemistry, and pharmacology of the genus Acer (maple): A review. J. Ethnopharm. 2016, 189, 31-60. [CrossRef] [PubMed]

8. Guo, H.; Li, B.-G.; Wu, Z.-J.; Zhang, G.-L. Lupane triterpenoids and a diarylheptanoid from Clematoclethra actinidioides. Planta Med. 2006, 72, 180-183. [CrossRef] [PubMed]

9. Ren, X.; He, T.; Chang, Y.; Zhao, Y.; Chen, X.; Bai, S.; Wang, L.; She, M.; She, G. The genus Alnus, a comprehensive outline of its chemical constituents and biological activities. Molecules 2017, 22, 1383. [CrossRef] [PubMed]

10. Rastogi, S.; Pandey, M.M.; Rawat, A.K.S. Medicinal plants of the genus Betula-Traditional uses and a phytochemical-pharmacological review. J. Ethnopharm. 2015, 159, 62-83. [CrossRef] [PubMed]

11. Masullo, M.; Cerulli, A.; Olas, B.; Pizza, C.; Piacente, S. Giffonin A-I, antioxidant cyclized diarylheptanoids from the leaves of hazelnut tree (Corylus avellana), source of the Italian PGI product "Nocciola di Giffoni". J. Nat. Prod. 2015, 78, 17-25. [CrossRef] [PubMed]

12. Masullo, M.; Cantone, V.; Cerulli, A.; Lauro, G.; Messano, F.; Russo, G.L.; Pizza, C.; Bifulco, G.; Piacente, S. Giffonin J-P, highly hydroxylated cyclized diarylheptanoids from the leaves of Corylus avellana cultivar “Tonda di Giffoni". J. Nat. Prod. 2015, 78, 2975-2982. [CrossRef] [PubMed]

13. Lee, J.S.; Kim, H.J.; Park, H.; Lee, Y.S. New diarylheptanoids from the stems of Carpinus cordata. J. Nat. Prod. 2002, 65, 1367-1370. [CrossRef] [PubMed]

14. Yasue, M.; Miyazaki, M.; Takahashi, T.; Imamura, H.; Honda, O. Wood extractives. VIII. Constituents of Ostrya japonica wood. J. Japan Wood Res. Soc. 1965, 11, 111-113.

15. Feng, M.-M.; Zhang, Y.-X.; Xia, B.; He, D.-H.; Ding, L.-S.; Zhou, Y.; Ye, X.-X. Chemical constituents in leaves of Ostryopsis nobilis and their antioxidant activities. Zhongyaocai 2013, 44, 2650-2656.

16. Zhang, Y.-X.; Xia, B.; Zhou, Y.; Ding, L.-S.; Peng, S.-L. Two new cyclic diarylheptanoids from the stems of Ostryopsis noblis. Chin. Chem. Lett. 2013, 24, 512-514. [CrossRef] 
17. Venkatraman, G.; Mishra, A.K.; Thombare, P.S.; Sabata, B.K. Diarylheptanoids from Garuga pinnata. Phytochemistry 1933, 33, 1221-1225. [CrossRef]

18. Ara, K.; Rahman, A.H.M.M.; Hasan, C.M.; Iskander, M.N.; Asakawa, Y.; Quang, D.N.; Rashid, M.A. Macrocyclic diarylheptanoids from Garuga pinnata. Phytochemistry 2006, 67, 2659-2662. [CrossRef] [PubMed]

19. Reddy, V.L.N.; Ravinder, K.; Srinivasulu, M.; Goud, T.V.; Reddy, S.M.; Srujankumar, D.; Rao, T.P.; Murty, U.S.; Venkateswarlu, Y. Two new macrocyclic diaryl ether heptanoids from Boswellia ovalifoliolata. Chem. Pharm. Bull. 2003, 51, 1081-1084. [CrossRef]

20. Kaneda, A.; Kinghorn, A.D.; Farnsworth, N.R.; Tuchinda, P.; Udchachon, J.; Santisuki, T.; Reutrakul, V. Two diarylheptanoids and a lignin from Casuarina junghuhniana. Phytochemistry 1990, 29, 3366-3368. [CrossRef]

21. Wu, H.-C.; Cheng, M.-J.; Peng, C.-F.; Yang, S.-C.; Chang, H.-S.; Lin, C.-H.; Wang, C.-J.; Chen, I.-S. Secondary metabolites from the stems of Engelhardia roxburghiana and their antitubercular activities. Phytochemistry 2012, 82, 118-127. [CrossRef] [PubMed]

22. Lee, K.-S.; Li, G.; Kim, S.H.; Lee, C.-S.; Woo, M.-H.; Lee, S.-H.; Jahng, Y.-D.; Son, J.-K. Cytotoxic diarylheptanoids from the roots of Juglans mandshurica. J. Nat. Prod. 2002, 65, 1707-1708. [CrossRef] [PubMed]

23. Li, G.; Xu, M.-L.; Choi, H.-G.; Lee, S.-H.; Jahng, Y.-D.; Lee, C.-S.; Moon, D.-C.; Woo, M.-H.; Son, J.-K. Four new diarylheptanoids from the roots of Juglans mandshurica. Chem. Pharm. Bull. 2003, 51, 262-264. [CrossRef] [PubMed]

24. Tanaka, T.; Jiang, Z.-H.; Isao, K. Distribution of ellagic acid derivatives and a diarylheptanoid in wood of Platycarya strobilacea. Phytochemistry 1998, 47, 851-854. [CrossRef]

25. Liu, H.B.; Cui, C.B.; Gu, Q.Q.; Zhang, D.Y.; Zhao, Q.C.; Guan, H.S. Pterocarine, a new diarylheptanoid from Pterocarya tonkinesis, its cell cycle inhibition at G0/G1 phase and induction of apoptosis in HCT-15 and K562 cells. Chin. Chem. Lett. 2005, 16, 215-218.

26. Jiang, Z.-H.; Tanaka, T.; Hirata, H.; Fukuoka, R.; Kouno, I. Three diarylheptanoids from Rhoiptelea chiliantha. Phytochemistry 1996, 43, 1049-1054. [CrossRef]

27. Whiting, D.; Wood, A. Cyclisation of 1,7-diarylheptanoids through oxidative, reductive, and photochemical radical processes: Total syntheses of the $m, m$-bridged biaryls myricanone and $( \pm)$-myricanol, and a related diaryl ether. Tetrahedron Lett. 1978, 19, 2335-2338. [CrossRef]

28. Inoue, T.; Arai, Y.; Nagai, M. Diarylheptanoids in the bark of Myrica rubra Sieb et. Zucc. Yakugaku Zasshi 1984, 104, 37-41. [CrossRef] [PubMed]

29. Makule, E.; Schmidt, T.J.; Heilmann, J.; Kraus, B. Diarylheptanoid glycosides of Morella salicifolia bark. Molecules 2017, 22, 2266. [CrossRef] [PubMed]

30. Tao, S.; Gao, G.; Qi, S.; Li, Q.; Zhang, C. Studies on the constituents of Scyphiphora hydrophyllacea (II). Zhongyaocai 2009, 32, 712-714. [PubMed]

31. Ma, X.-N.; Xie, C.-L.; Miao, Z.; Yang, Q.; Yang, X.-W. An overview of chemical constituents from Alpinia species in the last six decades. RSC Adv. 2017, 7, 14114-14144. [CrossRef]

32. Claeson, P.; Tuchinda, P.; Reutrakul, V. Naturally occurring 1,7-diarylheptanoids. J. Indian Chem. Soc. 1994, 71, 509-521.

33. Claeson, P.; Claeson, U.P.; Tuchinda, P.; Reutrakul, V. Occurrence, isolation, and bioactivity of 1,7-diarylheptanoids. Stud. Nat. Prod. Chem. 2002, 26, 881-908.

34. Lv, H.; She, G. Naturally occurring diarylheptanoids. Nat. Prod. Commun. 2010, 5, 1687-1708. [PubMed]

35. Lv, H.; She, G. Naturally occurring diarylheptanoids-A supplementary version. Rec. Nat. Prod. 2012, 6, 321-333.

36. Gupta, S.C.; Patchva, S.; Aggarwal, B.B. Therapeutic roles of curcumin: Lessons learned from clinical trials. AAPS J. 2013, 15, 195-218. [CrossRef] [PubMed]

37. Priyadarsini, K.I. The chemistry of curcumin: From extraction to therapeutic agent. Molecules 2014, 19, 20091-20112. [CrossRef] [PubMed]

38. Nelson, K.M.; Dahlin, J.L.; Bisson, J.; Graham, J.; Pauli, G.F.; Walters, M.A. The essential medicinal chemistry of curcumin: Miniperspective. J. Med. Chem. 2017, 60, 1620-1637. [CrossRef] [PubMed]

39. Rahman, A.F.M.M.; Lu, Y.; Lee, H.-J.; Jo, H.; Yin, W.; Alam, S.M.; Cha, H.; Kadi, A.A.; Kwon, Y.; Jahng, Y. Linear diarylheptanoids as potential anticancer therapeutics: Synthesis, biological evaluation, and structure-activity relationship studies. Arch. Pharm. Res. 2018. [CrossRef] 
40. Liu, J.X.; Di, D.L.; Wei, X.-N.; Han, Y. Cytotoxic diarylheptanoid from the pericarps of walnuts (Juglans regia). Planta Med. 2008, 74, 754-759. [CrossRef] [PubMed]

41. He, J.-B.; Yan, Y.-M.; Ma, X.-J.; Lu, Q.; Li, X.-S.; Su, J.; Li, Y.; Liu, G.-M.; Cheng, Y.-X. Sesquiterpenoids and diarylheptanoids from Nidus vespae and their inhibitory effects on nitric oxide production. Chem. Biodivers. 2011, 8, 2270-2276. [CrossRef] [PubMed]

42. Costantino, V.; Fattorusso, E.; Mangoni, A.; Perinu, C.; Teta, R.; Panza, E.; Ianaro, A. Tedarenes A and B: Structural and stereochemical analysis of two new strained cyclic diarylheptanoids from the marine sponge Tedania ignis. J. Org. Chem. 2012, 77, 6377-6383. [CrossRef] [PubMed]

43. Lee, M.-W.; Tanaka, T.; Nonaka, G.-I.; Hahn, D.-R. Phenolic compounds on the leaves of Betula platyphylla var. latifolia. Arch. Pharm. Res. 1992, 15, 211-214. [CrossRef]

44. Joshi, B.S.; Pelletier, S.W.; Newton, M.G.; Lee, D.; McGaughey, G.B.; Puar, M.S. Extensive 1D, 2D NMR spectra of some[7.0]metacyclophanes and X-ray analysis of ( \pm )-myricanol. J. Nat. Prod. 1996, 59, 759-764. [CrossRef]

45. Jones, J.R.; Lebar, M.D.; Jinwal, U.K.; Abisambra, J.F.; Koren III, J.; Blair, L.; O'Leary, J.C.; Davey, Z.; Trotter, J.; Johnson, A.G.; et al. The diarylheptanoid (+)-aR,11S-myricanol and two flavones from bayberry (Myrica cerifera) destabilize the microtubule-associated protein tau. J. Nat. Prod. 2011, 74, 38-44. [CrossRef] [PubMed]

46. Alberti, Á.; Riethmüller, E.; Béni, S. Characterization of diarylheptanoids: An emerging class of bioactive natural products. J. Pharm. Biomed. Anal. 2018, 147, 13-34. [CrossRef] [PubMed]

47. Yasue, M. Wood extractives. XI. Structure of asadanin. 3. J. Japan Wood Res. Soc. 1965, 11, 202-205.

48. Yasue, M. Wood extracts of Ostrya japonica. Structures of asadanin and related compounds. Ringyo Shikenjo Kenkyu Hokoku 1968, 209, 77-168.

49. Singldinger, B.; Dunkel, A.; Hofmann, T. The cyclic diarylheptanoid asadanin as the main contributor to the bitter off-taste in hazelnuts (Corylus avellana L.). J. Agric. Food Chem. 2017, 65, 1677-1683. [CrossRef] [PubMed]

50. Nomura, M.; Tokoroyama, T. Three new cyclized $\mathrm{C}_{9}-\mathrm{C}_{1}-\mathrm{C}_{9}$ compounds from Alnus japonica steud. J. Chem. Soc. Chem. Comm. 1974, 65-66. [CrossRef]

51. Nomura, M.; Tokoroyama, T. Further phenolic components from Alnus japonica. J. Chem. Soc. Chem. Comm. 1975, 316-317. [CrossRef]

52. Hanawa, F.; Shiro, M.; Hayashi, Y. Heartwood constituents of Betula maximowicziana. Phytochemistry 1997, 45, 589-595. [CrossRef]

53. Watanabe, N.; Sasaya, T. Extractives of the genus Corylus. II. Novel diarylheptanoids from the wood of Corylus sieboldiana Blume (1). J. Japan Wood Res. Soc. 1994, 40, 199-203.

54. Watanabe, N.; Sasaya, T.; Sano, Y. Extractives of the genus Corylus. III. Novel diarylheptanoids from the wood of Corylus sieboldiana blume. (2). J. Japan Wood Res. Soc. 1994, 40, 1219-1225.

55. Sung, S.H.; Lee, M. Anti-adipogenic activity of a new diarylheptanoid isolated from Alnus japonica on 3T3-L1 cells via modulation of PPAR $\gamma, \mathrm{C} / \mathrm{EBP} \alpha$ and SREBP1c signaling. Bioorg. Med. Chem. Lett. 2015, 25, 4648-4651. [CrossRef] [PubMed]

56. Fuchino, H.; Satoh, T.; Yokochi, M.; Tanaka, N. Chemical evaluation of Betula species in Japan. III. Constituents of Betula maximowicziana. Chem. Pharm. Bull. 1996, 44, 1748-1753. [CrossRef]

57. Nagumo, S.; Kaji, N.; Inoue, T.; Nagai, M. Studies on the constituents of Aceraceae plants. XI. Two types of cyclic diarylheptanoid from Acer nikoense. Chem. Pharm. Bull. 1993, 41, 1255-1257. [CrossRef]

58. Fuchino, H.; Satoh, T.; Tanaka, N. Chemical evaluation of Betula species in Japan. I. Constituents of Betula ermanii. Chem. Pharm. Bull. 1995, 43, 1937-1942. [CrossRef]

59. Nagumo, S.; Ishizawa, S.; Inoue, T.; Nagai, M. Studies on the constituents of Aceraceae plants. XIII. Diarylheptanoids and other phenolics from Acer nikoense. Chem. Pharm. Bull. 1996, 44, 1086-1089. [CrossRef]

60. Fuchino, H.; Konishi, S.; Satoh, T.; Yagi, A.; Saitsu, K.; Tatsumi, T.; Tanaka, N. Chemical evaluation of Betula species in Japan. II. Constituents of Betula platyphylla var. japonica. Chem. Pharm. Bull. 1996, 44, 1033-1038. [CrossRef]

61. Fuchino, H.; Satoh, T.; Hida, J.; Terada, M.; Tanaka, N. Chemical evaluation of Betula species in Japan. IV. Constituents of Betula davurica. Chem. Pharm. Bull. 1998, 46, 166-168. [CrossRef]

62. Wang, S.; Pei, Y. Diarylheptanoids from leaves of Betula platyphylla. Zhongcaoyao 2001, 32, 99-101. 
63. Takahashi, M.; Fuchino, H.; Sekita, S.; Satake, M. In vitro leishmanicidal activity of some scare natural products. Phytotherapy Res. 2004, 18, 573-578. [CrossRef] [PubMed]

64. Cerulli, A.; Lauro, G.; Masullo, M.; Cantone, V.; Olas, B.; Kontek, B.; Nazzaro, F.; Bifulco, G.; Piacente, S. Cyclic diarylheptanoids from Corylus avellana green leafy covers: Determination of their absolute configurations and evaluation of their antioxidant and antimicrobial activities. J. Nat. Prod. 2017, 80, 1703-1713. [CrossRef] [PubMed]

65. Chiba, K.; Ichizawa, H.; Kawai, S.; Nishida, T. $\alpha$-Glucosidase inhibition activity by cyclic diarylheptanoids from Alnus sieboldiana. J. Wood Chem. Tech. 2013, 33, 44-51. [CrossRef]

66. Inoue, T. Constituents of Acer nikoense and Myrica rubra. On diarylheptanoids. Yakugaku Zhazzhi 1993, 113, 181-197. [CrossRef]

67. Morikawa, T.; Tao, J.; Toguchida, I.; Matsuda, H.; Yoshikawa, M. Structures of new cyclic diarylheptanoids and inhibitors of nitric oxide production from Japanese folk medicine Acer nikoense. J. Nat. Prod. 2003, 61, 86-91. [CrossRef] [PubMed]

68. Campbell, R.V.M.; Crombie, L.; Tuck, B.; Whiting, D.A. Isolation and structure of new meta-bridged biphenyls from Myrica nagi. J. Chem. Soc. Chem. Comm. 1970, 1206-1207. [CrossRef]

69. Begley, M.J.; Whiting, D.A. X-ray study of 16-bromomyricanol; the structure of myricanol, a natural m,m-bridged bent biphenyl. J. Chem. Soc. Chem. Comm. 1970, 1207-1208. [CrossRef]

70. Begley, M.J.; Campbell, R.V.M.; Crombie, L.; Tuck, B.; Whiting, D.A. Constitution and absolute configuration of meta,meta-bridged stained biphenyls from Myrica nagi; X-ray analysis of 16-bromomyricanol. J. Chem. Soc. 1971, 3634-3642.

71. Martin, M.D.; Calcul, L.; Smith, C.; Jinwal, U.K.; Fontaine, S.N.; Darling, A.; Seeley, K.; Wojtas, L.; Narayan, M.; Gestwicki, J.E.; et al. Synthesis, stereochemical analysis, and derivatization of myricanol provide new probes that promote autophagic tau clearance. ACS Chem. Biol. 2015, 10, 1099-1109. [CrossRef] [PubMed]

72. Smyth, J.E.; Butler, N.M.; Keller, P.A. A twist of nature-The significance of atropisomers in biological systems. Nat. Prod. Rep. 2015, 32, 1562-1583. [CrossRef] [PubMed]

73. Cahn, R.S.; Ingold, C.; Prelog, V. Specification of molecular chirality. Angew Chem. Int. Ed. Engl. 1966, 5, 385-415. [CrossRef]

74. Anthonsen, T.; Lorentzen, G.B.; Malterud, K.E. Porson, a new [7,0]-metacyclophane from Myrica gale. Acta Chim. Scand. Ser. B 1975, 29, 529-530. [CrossRef]

75. Sun, D.W.; Zhao, Z.C.; Wong, H.; Foo, L.Y. Tannins and other phenolics from Myrica esculenta bark. Phytochemistry 1988, 27, 579-583.

76. Ren, Z.; Tong, Y.; Dai, G.; Chen, X.; Yang, F. Effect of myricanone on tumor in vitro. Zhonghua Zhongyiyao Xuekan 2014, 32, 2423-2425.

77. Dai, G.; Tong, Y.; Chen, X.; Ren, Z.; Yang, F. In vitro anticancer activity of myricanone in human lung adrenocarcinoma A549 cells. Chemotherapy 2014, 60, 81-87. [CrossRef] [PubMed]

78. Pual, A.; Das, S.; Das, J.; Samadder, A.; Bishayee, K.; Sadhukhan, R.; Bukhuda-bukhsh, A.R. Diarylheptanoid-myricanone isolated from ethanolic extract of Myrica cerifera showing anticancer effects on HeLa and PC3 cell lines: Signaling pathway and drug-DNA interaction. J. Integrative Med. 2013, 11, 405-415. [CrossRef] [PubMed]

79. Ting, Y.-C.; Ko, H.-H.; Wang, H.-C.; Peng, C.-F.; Chang, H.-S.; Hsieh, P.-C.; Chen, I.-S. Biological evaluation of secondary metabolites from the roots of Myrica adenophora. Phytochemistry 2014, 103, 89-98. [CrossRef] [PubMed]

80. Ishida, J.; Kozuka, M.; Tokuda, H.; Nishino, H.; Nagumo, S.; Lee, K.-H.; Nagai, M. Chemopreventive potential of cyclic diarylheptanoids. Bioorg. Med. Chem. 2002, 10, 3361-3365. [CrossRef]

81. Ibrahim, S.R.M.; Mohamed, G.A.; Khedr, A.I.M.; Aljaeid, B.M. Alnuheptanoid B: A new cyclic diarylheptanoid from Alnus japonica stem bark. Rec. Nat. Prod. 2016, 10, 362-368.

82. Wang, J.; Dong, S.; Wang, Y.; Lu, Q.; Zhong, H.; Du, G.; Zhang, L.; Cheng, Y. Cyclic diarylheptanoids from Myrica nana inhibiting nitric oxide release. Bioorg. Med. Chem. 2008, 16, 8510-8513. [CrossRef] [PubMed]

83. Yu, Y.-F.; Lu, Q.; Guo, L.; Mei, R.-Q.; Liang, H.-X.; Luo, D.-Q.; Cheng, Y.-X. Myricananone and myricananadiol: Two new cyclic diarylheptanoids from the roots of Myrica nana. Helv. Chim. Acta 2007, 90, 1691-1696. [CrossRef] 
84. Takeda, Y.; Fujita, T.; Shingu, T.; Ogimi, C. Studies on the bacterial gall of Myrica rubra: Isolation of a new [7.0]-metacyclophan from the gall and DL- $\beta$-phenylacetic acid from the culture of gall-forming bacteria. Chem. Pharm. Bull. 1987, 35, 2569-2573. [CrossRef]

85. Wang, J.-F.; Zhang, C.-L.; Lu, Q.; Yu, Y.-F.; Zhong, H.-M.; Long, C.-L.; Cheng, Y.-X. Three new diarylheptanoids from Myrica nana. Helv. Chim. Acta 2009, 92, 1594-1599. [CrossRef]

86. Matsuda, H.; Morikawa, T.; Tao, J.; Ueda, K.; Yoshikawa, M. Bioactive constituents of Chinese natural medicines. VII. 1: Inhibition of degranulation in RBL-2H3 cells and absolute stereo structures of three new diarylheptanoid glycosides from the bark of Myrica rubra. Chem. Pharm. Bull. 2002, 50, 208-215. [CrossRef] [PubMed]

87. Yaguchi, Y.; Sakura, N.; Nagai, M.; Inoue, T. Constituents of Myrica rubra. III. 1: Structures of two glycosides of myricanol. Chem. Pharm. Bull. 1988, 36, 1419-1424. [CrossRef]

88. Akazawa, H.; Fujita, Y.; Banno, N.; Watanabe, K.; Kimura, Y.; Manosrol, A.; Manosrol, J.; Akihisa, T. Three new cyclic diarylheptanoids and other phenolic compounds from the bark of Myrica rubra and their melanogenesis inhibitory and radical scavenging activities. J. Oleo. Sci. 2010, 59, 213-221. [CrossRef] [PubMed]

89. Yoshimura, M.; Yamakami, S.; Amakura, Y.; Yoshida, T. Diarylheptanoid sulfates and related compounds from Myrica rubra. J. Nat. Prod. 2012, 75, 1798-1802. [CrossRef] [PubMed]

90. Sakura, N.; Yaguchi, Y.; Hirakawa, T.; Nagai, M.; Inoue, T. Two myricanol glycosides from Myrica rubra and revision of the structure of isomyricanone. Phytochemistry 1991, 30, 3077-3079. [CrossRef]

91. Liu, J.X.; Di, D.L.; Huang, X.Y.; Li, C. Two new diarylheptanoids from the pericarps of Juglans regia L. Chin. Chem. Lett. 2007, 18, 943-946. [CrossRef]

92. Nagai, M.; Dohi, J.; Morihara, M.; Sakurai, N. Diarylheptanoids from Myrica gale var. tomentosa and revised structure of porson. Chem. Pharm. Bull. 1995, 43, 1674-1677. [CrossRef]

93. Krishnaswamy, S.; Pattabhi, V.; Gabe, E.J. Garuganin-II, an antibiotic. Acta Cryst. 1987, 43, 527-530. [CrossRef]

94. Navee, C.; Vijay-Kumar, S. Crystal structure of garuganin V, a tricyclononadecane from Garuga pinnata, $\mathrm{C}_{21} \mathrm{H}_{22} \mathrm{O}_{4}$. Zeit. Krist. 1995, 210, 365-366.

95. Ara, K.; Kaisar, M.A.; Rahman, M.S.; Chowdhury, S.R.; Islam, F.; Rashid, M.A. Antimicrobial constituents from Garuga pinnata Roxb. Latin Am. J. Pharm. 2012, 31, 1071-1073.

96. Wang, D.-Y.; Liu, G.-G. A new diarylheptanoid from the bark of Myrica nana. Nat. Prod. Res. 2008, 22, 292-295. [CrossRef] [PubMed]

97. Zeng, L.; Chen, L.; Liu, L. A new diarylheptanoid from the bark of Myrica nana. J. Chem. Res. 2009, 337-338. [CrossRef]

98. Moss, G.P. Basic terminology of stereochemistry. Pure Appl. Chem. 1996, 68, 2193-2222. [CrossRef]

99. Nagai, M.; Kubo, M.; Fujita, M.; Inoue, T.; Matsuo, M. Acerogenin A, a novel new cyclic diarylheptanoid. J. Chem. Soc. Chem. Comm. 1976, 338-339. [CrossRef]

100. Nagai, M.; Kubo, M.; Fujita, M.; Inoue, T.; Matsuo, M. Studies on the constituents of Aceraceae plants. II. Structure of acerosides I, a glycoside of novel cyclic diarylheptanoid from Acer nickoense Maxim. Chem. Pharm. Bull. 1978, 26, 2805-2810. [CrossRef]

101. Kubo, M.; Inoue, T.; Nagai, M. Studies on the constituents Aceraceae plants. III. Structure of acerogenin B from Acer nikoense Maxim. Chem. Pharm. Bull. 1980, 28, 1300-1302. [CrossRef]

102. Pretsch, E.; Clerc, T.; Seibl, J.; Simon, W. Tables of Spectrral Data for Structure Determination of Organic Compounds, 2nd ed.; Springer: Berlin, Germany, 1989.

103. Farnum, D.G.; Wilcox, C.F. Use of a model for the ring-current effect in analysis of the nuclear magnetic resonance spectra of di- and triphenylcyclopropenium ions. J. Am. Chem. Soc. 1967, 89, 5379-5383. [CrossRef]

104. Malterud, K.E.; Anthonsen, T.; Hjorta, J. 14-Oxa-[7.1]-metapara-cyclophane from Myrica gale L. A new class of natural products. Tetrahedron Lett. 1976, 17, 3069-3072. [CrossRef]

105. Morita, H.; Deguchi, J.; Motegi, Y.; Sato, S.; Aoyama, C.; Takeo, J.; Shiro, M.; Hirasawa, Y. Cyclic diarylheptanoids as $\mathrm{Na}^{+}$-glucose cotransporter (SLGT) inhibitors from Acer nikoense. Bioorg. Med. Chem. Lett. 2010, 20, 1070-1074. [CrossRef] [PubMed]

106. Kubo, M.; Nagai, M.; Inoue, T. Studies on the constituents Aceraceae plants. IV. Carbon-13 nuclear magnetic resonance spectra of acerogenin A, rhododendrol and related compounds, and structure of aceroside IV from Acer nikoense Maxim. Chem. Pharm. Bull. 1983, 31, 1917-1922. [CrossRef] 
107. Akihisa, T.; Taguchi, Y.; Yasukawa, K.; Tokuda, H.; Akazawa, H.; Suzuki, T.; Kimura, Y. Acerogenin M, a cyclic diarylheptanoid and other phenolic compounds from Acer nikoense and their anti-inflammatory and anti-tumor promoting effects. Chem. Pharm. Bull. 2006, 54, 735-739. [CrossRef] [PubMed]

108. Yonezawa, T.; Lee, J.-W.; Akazawa, H.; Inagaki, M.; Cha, B.-Y.; Nagai, K.; Yagasaki, K.; Akihisa, T.; Woo, J.-T. Osteogenic activity of diphenyl ether cyclic diarylheptanoids derived from Acer nikoense. Bioorg. Med. Chem. Lett. 2011, 21, 3248-3251. [CrossRef] [PubMed]

109. Akihisa, T.; Takeda, A.; Akazawa, H.; Kikuchi, T.; Yokokawa, S.; Ukiya, M.; Fukatsu, M.; Watanabe, K. Melanogenesis-inhibitory and cytotoxic activities of diaryheptanoids from Acer nikoense bark and their derivatives. Chem. Biodivers. 2012, 9, 1475-1489. [CrossRef] [PubMed]

110. Kim, N.-T.; Lee, D.-S.; Chowdhury, A.; Lee, H.; Cha, B.-Y.; Woo, J.-T.; Woo, E.R.; Jang, J.-H. Acergenin C from Acer nikoense exhibits a neuroprotective effect in mouse hippocampal HT22 cell lines through the upregulation of Nrf-2/HO-1 signaling pathways. Mol. Med. Rep. 2017, 16, 1537-1543. [CrossRef] [PubMed]

111. Deguchi, J.; Motegi, Y.; Nakata, A.; Hosoya, T.; Morita, H. Cyclic diarylheptanoids as inhibitors of NO production. J. Nat. Med. 2013, 67, 234-239. [CrossRef] [PubMed]

112. Morihara, M.; Sakurai, N.; Inoue, T.; Kawai, K.-I.; Nagai, M. Two novel diarylheptanoid glucosides Myrica gale var. tomentosa and absolute structure of plane-chiral galeon. Chem. Pharm. Bull. 1997, 45, 820-823.

113. Bryant, V.C.; Kishore Kumar, G.D.; Nyong, A.M.; Natarajan, A. Synthesis and evaluation of macrocyclic diarylether heptanoid natural products and their analogs. Bioorg. Med. Chem. Lett. 2012, 22, 245-248. [CrossRef] [PubMed]

114. Inoue, T.; Ishidate, Y.; Fuchita, M. Studies on the constituents of Aceraceae plants. I. Constituents in leaves and stem bark of Acer nikoense Maxim. Yakugaku Zasshi 1978, 98, 41-46. [CrossRef] [PubMed]

115. Nagai, M.; Kubo, M.; Takahashi, K. Studies on the constituents of Aceraceae plants. V. Two diaylheptanoid glycosides and an arylbutanol apiosylglucosiude from Acer nikoense. Chem. Pharm. Bull. 1983, 31, 1923-1928. [CrossRef]

116. Liang, J.; Peng, X.; Zhou, J.; Zhou, M.; Ruan, H. Diarylheptanoids from the fresh pericarps of Juglans sigillata. Nat. Prod. Res. 2018, 32, 2457-2463. [CrossRef] [PubMed]

117. Nagai, M.; Matshda, E.; Inoue, T.; Fujita, M.; Chi, H.J.; Ando, T. Studies on the constituents of Aceraceae plants. VII. Diarylheptanoids from Acer griseum and Acer triflorum. Chem. Pharm. Bull. 1990, 38, 1506-1508. [CrossRef]

118. Nagai, M.; Kenmochi, N.; Fujita, M.; Furukawa, N.; Inoue, T. Studies on the constituents of Aceraceae plants. VI. Revised stereochemistry of (-)-centrolobol, and new glycosides from Acer nikoense. Chem. Pharm. Bull. 1986, 34, 1056-1060. [CrossRef]

119. Pattawong, O.; Salih, M.Q.; Rosson, N.T.; Beaudry, C.M.; Cheong, P.H.-Y. The nature of persistent conformational chirality, racemization mechanisms, and predictions in diarylether heptanoid cyclophane natural products. Org. Biomol. Chem. 2014, 12, 3303-3309. [CrossRef] [PubMed]

120. Lin, W.-Y.; Peng, C.-F.; Tsai, I.-L.; Chen, J.-J.; Cheng, M.-J.; Chen, I.-S. Antitubercular constituents from the roots of Engelhardia roxburghiana. Planta Med. 2005, 71, 171-175. [CrossRef] [PubMed]

121. Li, G.; Lee, S.-Y.; Lee, K.-S.; Lee, S.-W.; Kim, S.-H.; Lee, S.-H.; Lee, C.-S.; Woo, M.-H.; Son, J.-K. DNA topoisomerase I and II inhibitory activity of constituents isolated from Juglans mandshuria. Arch. Pharm. Res. 2003, 26, 466-470. [CrossRef] [PubMed]

122. Kalchhauser, H.; Krishnamurty, H.G.; Talukdar, A.C.; Schmid, W. Isolation and structure determination of two new macrocyclic biaryl ethers from Garuga gamblei. Monatsh. Chem. 1988, 119, 1047-1051. [CrossRef]

123. Nethaji, M.; Pattabhi, V.; Krishnamurthy, H.G.; Talukdar, A.C. Structures of garugamblin-I and garubamblin-II: Two natural ansa bridged biaryl ethers. Acta Cryst. C Cryst. Struct. Comm. 1990, 46, 307-310. [CrossRef]

124. Kang, H.-M.; Son, K.-H.; Yang, D.C.; Han, D.C.; Kim, J.H.; Baek, N.-I.; Kwon, B.-M. Inhibitory activity of diarylheptanoids on farnesyl protein transferase. Nat. Prod. Res. 2004, 18, 295-299. [CrossRef] [PubMed]

125. Pattabhi, V.; Krishnaswami, S.; Gabe, E.J. Garuganin-I, an antibiotic, $\mathrm{C}_{22} \mathrm{H}_{24} \mathrm{O}_{5}$. Acta Cryst. 1984, 40, $832-834$. [CrossRef]

126. Haribal, M.M.; Mishra, A.K.; Sabata, B.K. Isolation and structure of a new macrocyclic, 15-membered biphenyl ether. Garuganin-I from Garuga pinnata. Tetrahedron 1985, 41, 4949-4951. [CrossRef]

127. Mishra, A.K.; Haribal, M.M.; Sabata, B.K. Garuganin III, a macrocyclic biphenyl ether from Garuga pinnata. Phytochemistry 1985, 24, 2463-2464. [CrossRef] 
128. Keserü, G.M.; Dienes, Z.; Nógrádi, M.; Kajtár-Peredy, M. Synthesis and revised structure of garuganin III. J. Org. Chem. 1993, 58, 6725-6728. [CrossRef]

129. Zhu, Z.-Q.; Beaudry, C.M. Structural revision of garuganin IV and 1,9'-didesmethylgaruganin III through total synthesis. J. Org. Chem. 2013, 78, 3336-3341. [CrossRef] [PubMed]

130. Zhu, Z.-Q.; Salih, M.Q.; Fynn, E.; Bain, A.D.; Beaudry, C.M. The garuganin and garugamblin diarylether heptanoids: Total synthesis and determination of chiral properties using dynamic NMR. J. Org. Chem. 2013, 78, 2881-2896. [CrossRef] [PubMed]

131. Fuchino, H.; Satoh, T.; Yokochi, M.; Tanaka, N. Chemical evaluation of Betula species in Japan. V. Constituents of Betula ovalifolia. Chem. Pharm. Bull. 1998, 46, 169-170. [CrossRef]

132. Masullo, M.; Mari, A.; Cerulli, A.; Bottone, A.; Kontek, B.; Olas, B.; Pizza, C.; Piacente, S. Quali-quantitative analysis of the phenolic fraction of the flowers of Corylus avellana, source of the Italian PGI product “Nocciola di Giffoni": Isolation of antioxidant diarylheptanoids. Phytochemistry 2016, 130, 273-281. [CrossRef] [PubMed]

133. Singldinger, B.; Dunkel, A.; Bahmann, D.; Bahmann, C.; Kadow, D.; Bisping, B.; Hofmann, T. New taste-active 3-(O- $\beta$-D-glucosyl)-2-oxoindole-3-acetic acids and diarylheptanoids in Cimiciato-infected hazelnuts. J. Agric. Food Chem. 2018, 66, 4660-4673. [CrossRef] [PubMed]

134. Eliel, E.I. Stereochemistry of Carbon Compounds; McGraw-Hill: New York, NY, USA, 1962; p. 156.

135. Petit, M.; Lapierre, A.J.B.; Curran, D.P. Relaying asymmetry of transition atropisomers of $o$-iodoanilides by radical cyclization. J. Am. Chem. Soc. 2005, 127, 14994-14995. [CrossRef] [PubMed]

136. Geissman, T.A.; Crout, D.H.G. Organic Chemistry of Secondary Plant. Products; Freeman Cooper: San Francisco, CA, USA, 1969.

137. Roughley, P.J.; Whiting, D.A. Diarylheptanoids: The problems in the biosynthesis. Tetrahedron Lett. 1971, 12, 3741-3746. [CrossRef]

138. Inoue, T.; Kenmochi, N.; Furukawa, N.; Fujita, M. Biosynthesis of acerogenin A, a diarylheptanoid from Acer nikoense. Phytochemistry 1987, 26, 1409-1411. [CrossRef]

139. Miyaura, N.; Suginome, H.; Suzuki, A. A stereospecific synthesis of conjugated $(E, Z)$ - and $(Z, Z)$-alkadienes by a palladium-catalyzed cross coupling reaction of 1-alkenoboranes with 1-alkenyl halides. Tetrahedron Lett. 1981, 22, 127-130. [CrossRef]

140. Sheffy, F.K.; Stille, J.K. Palladium-catalyzed coupling of allyl halides with organotins. J. Am. Chem. Soc. 1983, 105, 7173-7175. [CrossRef]

141. Negishi, E.; King, A.O.; Okukado, N. Selective carbon-carbon bond formation via transition metal catalysis. 3. A highly selective synthesis of unsymmetrical biaryls and diarylmethanes by the nickelor palladium-catalyzed reaction of aryl- and benzylzinc derivatives with aryl halides. J. Org. Chem. 1977, 42, 1821-1823. [CrossRef]

142. Ullmann, F.; Sponagel, P. Über die Phenylirung von Phenolen. Ber. Dtsch. Chem. Ges. 1905, 38, $2211-2212$. [CrossRef]

143. Lindley, J. Copper assisted nucleophilic substitution of aryl halogen. Tetrahedron 1984, 40, $1433-1456$. [CrossRef]

144. Bergley, W.J.; Grimshaw, J. Synthesis of nitidine (8,9-dimethoxy-5-methyl-2,3methylenedioxybenzo[c]phenanthridinium): A comparison of electrochemical and photochemical methods. J. Chem. Soc. Perkin Trans. 1 1977, 2324-2328. [CrossRef]

145. Wittig, G.; Geissler, G. Course of reactions of pentaphenyl phosphorous and certain derivatives. Ann. Chem. 1953, 580, 44-57. [CrossRef]

146. Moore, C.W.; Thorpe, J.F. The formation and reactions of imino-compounds (VI). The formation of derivatives of hydridene from o-phenylenediacetonitrile. J. Chem. Soc. Trans. 1908, 93, 165-187. [CrossRef]

147. Grubbs, R.H.; Brunck, T.K. Possible intermediate in the tungsten-catalyzed olefin metathesis reaction. J. Am. Chem. Soc. 1972, 94, 2538-2540. [CrossRef]

148. Dansou, B.; Pichon, C.; Dhal, R.; Brown, E.; Mille, S. Isolation of macrocyclic metacyclophanes from the attempted synthesis of [7.0]metacyclophanes of the myricanone series by Thorpe-Ziegler-intramolecular cyclization of diarys substituted by $\omega$-cyanoalkyl chains. Eur. J. Org. Chem. 2000, 1527-1533. [CrossRef]

149. Semmelhack, M.F.; Ryono, L. Nickel-promoted synthesis of cyclic biphenyls. Total synthesis of alnusone dimethyl ether. J. Am. Chem. Soc. 1975, 97, 3873-3875. [CrossRef] 
150. Semmelhack, M.F.; Helquist, P.; Jones, L.D.; Keller, L.; Mendelson, L.; Ryono, L.S.; Smith, J.G.; Stauffer, R.D. Reaction of aryl and vinyl halides with zerovalent nickel-Preparative aspects and the synthesis of alnusone. J. Am. Chem. Soc. 1981, 103, 6460-6471. [CrossRef]

151. Whiting, D.; Wood, A. Total synthesis of the meta,meta-bridged biphenyls ( \pm )-myricanol and myricanone, and of an isomeric biphenyl ether, a 14-oxa[7.1]metaparacyclophane. J. Chem. Soc. Perkin Trans. 1 1980, 623-628. [CrossRef]

152. Henley-Smith, P.; Whiting, D.A.; Wood, A.F. Methods for the construction of linear 1,7-diarylheptanoids: Synthesis of di-O-methylcentrolobol and precursors (synthetic and biosynthetic) to the meta,meta-bridged biphenyls myricanol and myricanone. J. Chem. Soc. Perkin 1 1980, 614-622. [CrossRef]

153. Ogura, T.; Usuki, T. Total synthesis of acerogenin E, G, and K, and centrolobol. Tetrahedron 2013, 69, $2807-2815$. [CrossRef]

154. Carbonnelle, A.-C.; Zhu, J. A novel synthesis of biaryl-containing macrocycles by a domino Miyaura arylboronate formation: Intramolecular Suzuki reaction. Org. Lett. 2000, 2, 3477-3480. [CrossRef] [PubMed]

155. Darzi, E.R.; White, B.M.; Loventhal, L.K.; Zakharov, L.V.; Jasti, R. An operationally simple and mild oxidative homocoupling of aryl boronic esters to access conformationally constrained macrocycles. J. Am. Chem. Soc. 2017, 139, 3106-3114. [CrossRef] [PubMed]

156. Riley, A.P.; Prisinzano, T.E. Studies towards the Total Synthesis of (+)-aR,11S-Myricanol. In Proceedings of the 244th ACS National Meeting \& Exhibition, Philadelphia, PA, USA, 19-23 August 2012.

157. Weyer, M.J.; Nye, J.; Ostlund, A.J.; Cook, G.R. Total Synthesis of the Diarylheptanoid (+)-aR,11S-Myricanol. In Proceedings of the 245th ACS National meeting \& Exhibition, New Orleans, LA, USA, 7-11 April 2013.

158. Rybak, T.; Hall, D. Stereoselective and regiodivergent allylic Suzuki-Miyaura cross coupling of 2-ethoxydihydroxypyranyl boronates: Synthesis and confirmation of absolute stereochemistry of diospongin B. Org. Lett. 2015, 17, 4156-4159. [CrossRef] [PubMed]

159. Yamamura, S.; Nishiyama, S. Biomimetic synthesis of isodityrosine natural products, and an approach to chemistry and molecular recognition of secoaglucovancomycin and related oligopeptides. J. Syn. Org. Chem. Jpn. 1997, 55, 1029-1039. [CrossRef]

160. Zhu, J. $\mathrm{S}_{\mathrm{N}}$ Ar bases macrocyclization via biaryl ether formation: Application in natural product synthesis. Synlett 1997, 2, 133-144. [CrossRef]

161. Cava, M.P.; Afzali, A. Practical route to bisbenzylisoquinolines by an improved Ullmann diphenyl ether synthesis. J. Org. Chem. 1975, 40, 1553-1556. [CrossRef]

162. Sambiagio, C.; Marsden, S.P.; Blacker, A.J.; McGowan, P.C. Copper catalyzed Ullmann type chemistry: From mechanistic aspects to modern development. Chem. Soc. Rev. 2014, 43, 3525-3550. [CrossRef] [PubMed]

163. Sawyer, J.C. Recent advances in diary ether synthesis. Tetrahedron 2000, 56, 5045-5065. [CrossRef]

164. Pitsinos, E.N.; Vivaldi, V.P.; Couladouros, E.A. Diaryl ether formation in the synthesis of natural products. Eur. J. Org. Chem. 2011, 1207-1222. [CrossRef]

165. Salih, M.Q.; Beaudry, C.M. Bio-inspired oxidative phenolic coupling: Total synthesis of the diarylether heptanoids ( \pm )-pterocarine. Tetrahedron Lett. 2017, 58, 2023-2025. [CrossRef]

166. Omura, K. Rapid conversion of phenols to $p$-benzoquinones under acidic conditions with lead oxide. Synthesis 1998, 145-1148.

167. Gonzalez, G.I.; Zhu, J. First total synthesis of acerogenin C and aceroside IV. J. Org. Chem. 1997, 62, 7544-7545. [CrossRef]

168. Meisenheimer, J. Über Reactionen aromatischer Nitrokörper. Ann. Chem. 1902, 323, 205-246. [CrossRef]

169. Doyle, M.P.; Dellaria, J.F.; Siegfried Jr., B.; Bishop, S.W. Reductive deamination of arylamines by alkyl nitriles in $\mathrm{N}, \mathrm{N}$-dimethylformamide. A direct conversion of arylamines to aromatic hydrovarbons. J. Org. Chem. 1977, 42, 3494-3498. [CrossRef]

170. Gonzalez, G.I.; Zhu, J. A unified strategy toward the synthesis of acerogenin-type macrocycles: Total syntheses of acerogenins A, B, L, and aceroside IV. J. Org. Chem. 1999, 64, 914-924. [CrossRef] [PubMed]

171. Keserü, G.M.; Nógrádi, M.; Szöllösy, Á. Synthesis of acerogenin C and (+)-acerogenin A, two macrocyclic diarylheptanoid constituents of Acer nikoense. Eur. J. Org. Chem. 1998, 521-524. [CrossRef]

172. Jeong, B.-S.; Wang, Q.; Son, J.-K.; Jahng, Y. A versatile synthesis of cyclic diphenyl ether-type diarylheptanoids: Acerogenins, ( \pm )-galeon, and $( \pm)$-pterocarine. Eur. J. Org. Chem. 2007, 1338-1344. [CrossRef] 
173. Wang, Q.; Son, J.-K.; Jahng, Y. First total synthesis of cytotoxic diarylheptanoids, galeon and pterocarine. Synth. Comm. 2007, 37, 675-681. [CrossRef]

174. Shen, L.; Sun, D. Total synthesis and structural revision of engelhardione. Tetrahedron Lett. 2011, 52, 4570-4574. [CrossRef] [PubMed]

175. Salih, M.Q.; Beaudry, C.M. Chirality in diarylether heptanoids; Synthesis of myricatomentogenin, jugcathanin, and congers. Org. Lett. 2012, 14, 4026-4029. [CrossRef] [PubMed]

176. Kozikowski, A.P.; Tuckemantel, W. The total synthesis of 2,3,3a,4,5,7a-hexahydro-1H-inden-1-ols by intramolecular Diels-Alder reactions of 1,3,8-nonatriene-5-ols. Dependence of product stereochemistry on the substitution pattern. J. Org. Chem. 1991, 56, 2826-2837. [CrossRef]

177. Vermes, B.; Keserü, G.M.; Mezey-Vándor, G.; Nógrádi, M.; Tóth, G. The total synthesis of garugamblin 1. Tetrahedron 1993, 49, 4893-4900. [CrossRef]

178. Keserü, G.M.; Nógrádi, M.; Kajtár-Peredy, M. Synthesis garugamblin-2, a macrocyclic diaryheptanoid constituents of Garuga gamblei. Liebigs Ann. Chem. 1994, 361-364. [CrossRef]

179. Kumar, G.D.K.; Natarajan, A. Total synthesis of ovalifoliolatin B, acerogenins A and C. Tetrahedron Lett. 2008, 49, 2103-2105. [CrossRef]

180. Salih, M.Q.; Beaudry, C.M. Enantioselective Ullmann ether couplings: Syntheses of (-)-myricantomentogenin, (-)-jugcathanin, (+)-galeon, and pterocarine. Org. Lett. 2013, 15, 4540-4543. [CrossRef] [PubMed]

181. Ding, Q.; Wang, Q.; He, H.; Cai, Q. Asymmetric synthesis of (-)-pterocarine and (-)-galeon via chiral phase transfer-catalyzed atropselective formation of diarylether cyclophane skeleton. Org. Lett. 2017, 19, 1804-1807. [CrossRef] [PubMed]

182. Ishida, J.; Ohtsu, A.; Tachibana, Y.; Nakanishi, Y.; Bastow, K.F.; Nagai, M.; Wang, H.-K.; Itokawa, H.; Lee, K.-H. Antitumor agents. Part 214: Synthesis and evaluation of curcumin analogues as cytotoxic agents. Bioorg. Med. Chem. 2002, 10, 3481-3487. [CrossRef]

183. Shen, L.; Maddox, M.M.; Adhikari, S.; Bruhn, D.F.; Kumar, M.; Lee, R.E.; Hurdle, J.G.; Lee, R.E.; Sun, D. Syntheses and evaluation of macrocyclic engelhardione analogs as antitubercular and antibacterial agents. J. Antiobiot. 2013, 66, 319-325. [CrossRef] [PubMed]

Sample Availability: Samples of the compounds 25b, 26a, 90, 96, 97, 98, and 99 are available from the authors.

(C) 2018 by the authors. Licensee MDPI, Basel, Switzerland. This article is an open access article distributed under the terms and conditions of the Creative Commons Attribution (CC BY) license (http:// creativecommons.org/licenses/by/4.0/). 